\title{
Adoption of Additive Manufacturing Processes toward 3D Printed Electronics
}

\author{
by \\ Abdurrazag Elaskri \\ A thesis submitted to the Faculty of Graduate Studies and Research \\ in partial fulfillment of the requirements for the degree of \\ Master of Applied Science \\ in \\ Mechanical Engineering \\ Ottawa-Carleton Institute for Mechanical and Aerospace Engineering \\ Department of Mechanical Engineering \\ Carleton University, \\ Ottawa, Ontario, Canada
}

Copyright $(\odot$

Abdurrazag Elaskri , 2021 


\section{Acknowledgments}

During the writing of this dissertation, I have got a great deal of assistance and support. Firstly, I would like to thank my supervisor, Professor Alex Ellery, whose expertise was invaluable in formulating the research questions and methodology. You provided me with the tools to choose the right direction and completed my dissertation.

My special thanks to my wife Mashaaer and my children Safie, Basima, Wala and Ansam for their love, support, patience and encouragement during my studies. Further I would like to acknowledge the jury members of this thesis. You provided very useful hints for improving this thesis manuscript, which I have gladly embraced. Finally, and most importantly, I am extremely thankful to my parents, Mehdi Elaskri and Raja Krifa, for their guidance and support. You are always there for me. 


\begin{abstract}
This research will present progress in developing the 3D printing of an electric motor. It takes advantage of the unique design freedom provided by Additive Manufacturing (AM), which has the potential to develop the way electrical motors are manufactured.

Recently, researchers have focused on increasing the capability of using AM to fabricate parts with the integration of electronic components. Several research groups have reported embedding electronic components into 3D printed objects during process interruptions. Though, to date, there is a lack of fabricated devices with electromechanical functionality for parts entirely manufactured using only AM technologies.

This study provides a new and different approach to creating fully 3D printed electric motors, focusing on an additively manufactured iron core, winding, insulation, and permanent magnet. In particular, we investigated how to develop a 3D printed motor with multi-material and a multi-technique to achieve a fully $3 \mathrm{D}$ printed motor that can be build without embedding devices.

Several prototypes of radial and axial flux DC electric motors were fabricated by using different 3D printing techniques, including Fused Deposition Modelling (FDM), Cold Spray Additive Manufacturing (CSAM), and Laser Powder Bed Fusion (LPBF). The Laminated Object Manufacturing (LOM) principles were also used to form the winding that was made of an electrically conductive material. The angular velocity and torque of the 3D printed motors were measured to specify the motors' performance. These motors were built using different materials, including polylactic acid (PLA), acrylonitrile butadiene styrene (ABS), Magnetic Iron PLA, silicon steel (Fe-3Si), and silicon steel (Fe-6.5Si)).

This study has demonstrated the possibility of fabricating a fully 3D printed DC electric motor in which only the copper foil was extracted in a LOM-like process. The coils were replaced by copper foil, which can be 3D-printed using the LOM technique (though this requires further investigation to determine the efficacy of this technique). All parts of the electric motors were fabricated with 3D-printing techniques, and the functionality indicated the potential of achieving a fully self-assembled 3D-printed motor using inexpensive, commercially available equipment. The 3D printed motors in this project are a modest step towards demonstrating that AM/3D printing as a process constitutes a universal construction mechanism from which a self-replicating machine can be built. Suppose such a machine was deployed on the moon; for example, in that case, it could be a robotic system that uses lunar materials to replicate itself and, ultimately, construct a wide range of future space assets, thereby demonstrating that 3D-printing could lead to significant and sustainable autonomous operations in space exploration.
\end{abstract}




\section{Publications}

Part of the work completed has been presented and published in multiple well-known conferences, as mentioned below.

1. A. Elaskri, A. Ellery. Developing techniques to 3d print electric motors. International Symposium on Artificial Intelligence, Robotics and Automation in Space (i-SAIRAS 2018), Madrid, Spain, 2018. (Conference Contribution - Full conference paper).

2. A. Ellery, A. Elaskri. Steps toward self-assembly of lunar structures from modules of 3d-printed in-situ resources. 70th International Astronautical Congress (IAC), Washington D.C., United States, 2019. (Conference Contribution - Full conference paper).

3. A. Elaskri, A. Ellery. $3 d$ printed electric motors as a step towards self-replicating machines. International Symposium on Artificial Intelligence, Robotics and Automation in Space (i-SAIRAS 2020). Pasadena, California. October 21, 2020. (Accepted- Full conference paper) 


\section{Contents}

$\begin{array}{lll}\text { Acknowledgments } & \text { i }\end{array}$

$\begin{array}{lll}\text { List of Acronyms } & \mathbf{v}\end{array}$

1 Introduction 1

1.1 Research Scope, Aim, Novelty, and Objectives . . . . . . . . . . . . . . . . . 2

1.1.1 Research Scope . . . . . . . . . . . . . . . . . . . 2

1.1 .2 Research Aim . . . . . . . . . . . . . . . . . . . . . . . . 3

1.1.3 Research Novelty . . . . . . . . . . . . . . . . . . . . . . . . 3

1.1.4 Research Objectives . . . . . . . . . . . . . . . . . . . . 3

2 Literature Review $\quad 5$

2.1 History and Application of Additive Manufacturing $\ldots \ldots \ldots \ldots \ldots \ldots$

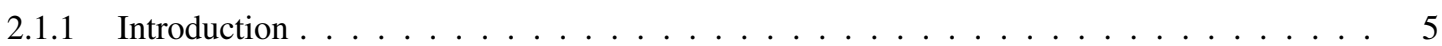

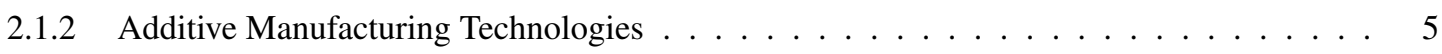

2.1.3 Tradeoffs between AM Processes $\ldots \ldots \ldots \ldots \ldots$

2.1.3.1 Fused Deposition Modeling $(F D M) \ldots \ldots \ldots \ldots$

2.1.3.2 Laminated Object Manufacturing $(L O M) \ldots \ldots \ldots \ldots$

2.1.3.3 Laser Powder Bed Fusion (LPBF) . . . . . . . . . . . . . . . . . . . . . 14

2.1.3.4 Cold Spraying Additive Manufacturing (CSAM) . . . . . . . . . . . . . 16

2.2 Elements of Electric Motor Construction and Design _ . . . . . . . . . . . . . . . . 19

2.2.1 Electric Motor Construction _. . . . . . . . . . . . . . . . . . . . 19

2.2.2 Working Principles of Brushed Permanent DC Motor . . . . . . . . . . . . . . 21

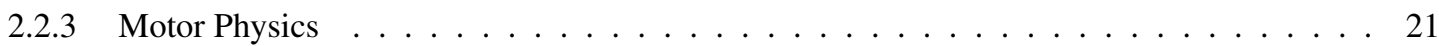

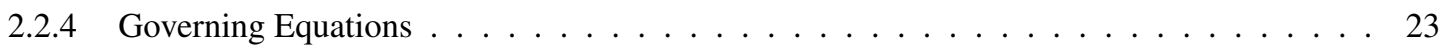




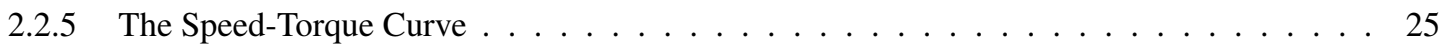

2.2.6 Friction and Motor Efficiency . . . . . . . . . . . . . . . . 27

2.2.7 Experimentally Characterizing a Brushed DC Motor ～. . . . . . . . . . . . . . . . 27

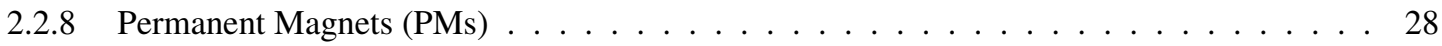

2.2.8.1 Magnetization Process . . . . . . . . . . . . . . . . . . . . 31

2.2.8.2 Potential for Permanent Magnet Production Using 3D printing Technologies . . 34

2.2.9 Soft Magnetic Cores _ . . . . . . . . . . . . . . . . . . . . 37

2.2.9.1 Silicon steel $(\mathrm{Fe}-\mathrm{Si}) \quad \ldots \ldots \ldots$

2.2.9.2 Iron-Cobalt alloys $(\mathrm{Fe}-\mathrm{Co}) \quad \ldots \ldots \ldots$. . . . . . . . . . . . 39

2.2.9.3 Nickel-Iron alloys $(\mathrm{Fe}-\mathrm{Ni}) \quad \ldots \ldots \ldots$. . . . . . . . . . . . . . . 40

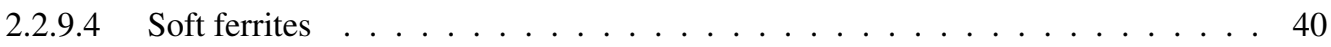

2.2.9.5 Soft magnetic composites $(\mathrm{SMCs}) \ldots \ldots \ldots \ldots$. . . . . . . . . 40

2.2.9.6 Amorphous Alloys (or Metallic Glasses) _ . . . . . . . . . . . . . . . . 41

2.2.9.7 Electromagnetization Process . . . . . . . . . . . . . . . . . . . 42

2.2.9.8 Potential for Motor Core Production Using AM Technologies . . . . . . . . . . 43

2.3 Relevant Previous Attempts to 3D Print Functional Objects . . . . . . . . . . . . . . . 44

2.4 Research contributions . . . . . . . . . . . . . . . . . . . . . . . . 45

3 Methodology: Fabricating and Testing of Radial-Flux DC Motor 46

3.1 Materials and Methods Used in the Manufacture of Rotor Core . . . . . . . . . . . . . . . . 47

3.1.1 Results and Discussion . . . . . . . . . . . . . . . . 48

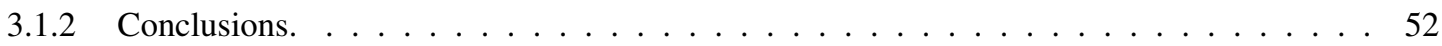

3.2 Materials and Methods Used in the Manufacture of Stator Core . . . . . . . . . . . . . . 52

3.2.1 Stator core made of Soft Iron $(\mathrm{Fe} 99.9 \%) \ldots \ldots \ldots \ldots \ldots$

3.2.1.1 Results and Discussion . . . . . . . . . . . . . . . . 54

3.2.2 Stator Core Made of Maraging Steel $(\mathrm{MS} 1) \ldots \ldots \ldots \ldots \ldots$

3.2.2.1 Results and Discussion . . . . . . . . . . . . . . . . . 57

3.2.3 Stator Core Made of Magnetic Iron PLA $\ldots \ldots \ldots$

3.2.3.1 Results and Discussion $\ldots \ldots \ldots$. . . . . . . . . . . . . . 59

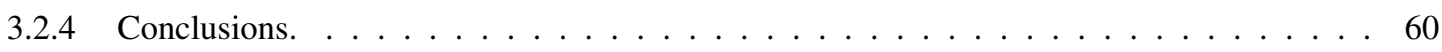


4.1 Materials and Methods Used in the Manufacture of Motor Rotor Made of Copper Coil Winding

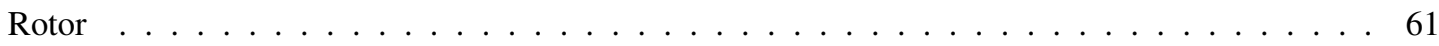

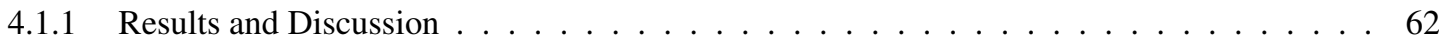

4.2 Materials and Methods Used in the Manufacture of Motor Rotor Made of Printed Circuit Board

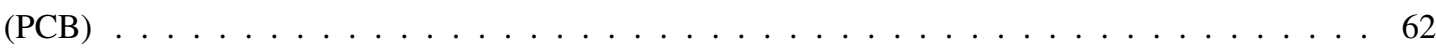

4.2.1 Motor Rotor Made of Single-Sided Printed Circuit Board (PCB) . . . . . . . . . . . . . . 62

4.2.2 Motor Rotor Made of Double-Sided Printed Circuit Board (PCB) . . . . . . . . . . . . . 65

4.2 .3 Results and Discussion . . . . . . . . . . . . . . . . . . . 67

4.3 Materials and Methods Used in the Manufacture of Motor Rotor Made of Conductive Copper

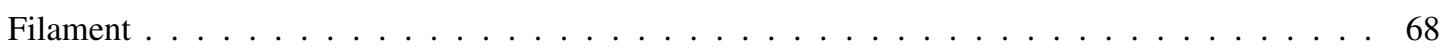

4.3.1 Results and Discussion . . . . . . . . . . . . . . . . . . . 69

4.4 Materials and Methods Used in the Manufacture of Motor Rotor Made Using Laminated Object Manufacturing Like Process . . . . . . . . . . . . . . . . . . . 70

4.4.1 Results and Discussion . . . . . . . . . . . . . . . . . . 74

4.5 Materials and Methods Used in the Manufacture of Fully 3D Printed Radial-Flux DC Motor . . . 74

4.5 .1 Results and Discussion . . . . . . . . . . . . . . . . . . 77

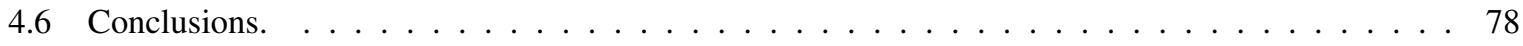

$5 \quad$ Validate Performance and Benchmarking 79

5.1 Motor Design . . . . . . . . . . . . . . . . . . . . . . . 80

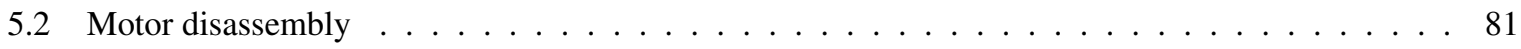

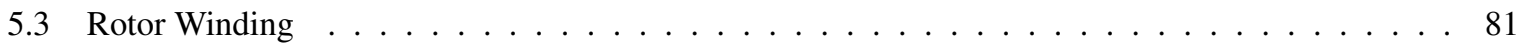

5.4 Motor Dimensions . . . . . . . . . . . . . . . . . . . . . . . 82

5.5 Motor Redesign . . . . . . . . . . . . . . . . . . . . . . . 82

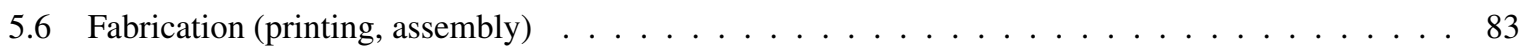

5.7 Results and Discussion . . . . . . . . . . . . . . . . . . . . 85

5.8 Conclusions. . . . . . . . . . . . . . . . . . . . . . . 87

6 Conclusions and Future Work $\quad 89$

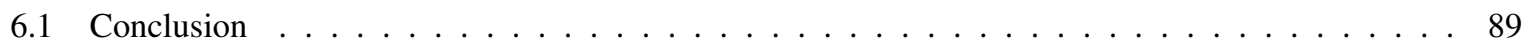

6.2 Future Work . . . . . . . . . . . . . . . . . . . . . . . . 90 


\section{List of Acronyms}

\begin{tabular}{|c|c|}
\hline Acronym & Definition \\
\hline ABS & Acrylonitrile Butadiene Styrene \\
\hline $\mathrm{AM}$ & Additive Manufacturing \\
\hline BDCM & Brushed DC Motor \\
\hline BJ & Binder Jetting \\
\hline CAD & Computer-Aided Design \\
\hline CSAM & Cold Spray Additive Manufacturing \\
\hline DED & Directed Energy Deposition \\
\hline DLP & Direct Light Processing \\
\hline DTM & Desktop Manufacturing \\
\hline Dyno & Motor Dynamometer \\
\hline EMF & Electromagnetic Field \\
\hline ESC & Electronic Speed Control \\
\hline FDM & Fused Deposition Modeling \\
\hline HIPS & High-Impact Polystyrene \\
\hline LOM & Laminated Object Manufacturing \\
\hline LPBF & Laser Powder Bed Fusion \\
\hline NRC & National Research Council of Canada \\
\hline PA & Aliphatic PolyAmides \\
\hline PCB & Printed Circuit Board \\
\hline PLA & PolyLactic Acid \\
\hline PMs & Permanent Magnets \\
\hline RepRap & Replicating Rapid Prototyper \\
\hline $\mathrm{RE}$ & Reverse Engineering \\
\hline $\mathrm{RP}$ & Rapid Prototyping \\
\hline SLA & Stereolithography Apparatus \\
\hline SLS & Selective Laser Sintering \\
\hline STL & A file Format Native to the Stereolithography CAD Software \\
\hline
\end{tabular}




\begin{tabular}{|l|l|}
\hline Acronym & Definition \\
\hline TPU & Thermoplastic PolyUrethane \\
\hline
\end{tabular}




\section{Chapter 1}

\section{Introduction}

Fabrication of functional objects utilizing Additive Manufacturing (AM) is a new research area. The basic approach is to embed off-the-shelf devices in a printed object by inserting conductive wires into the printed geometry during or after printing. In recent studies, conductive inks were also used to form electronic circuits into 3D printed parts [1]. As an example, Lopes et al. [2] described the process in which the 3D printed part was embedded with electrical components and electronic circuits made of conductive ink.

Regardless of the employment of AM technologies for producing a wide range of dielectric and metallic materials, previous studies about fabricating electromechanical devices are relatively limited. All attempts to 3D-print electric motors only considered the main structure of the rotor and stator. The little research on the capabilities of $\mathrm{AM}$ on the development of products with mixed materials and different technologies, such as circuit boards and electric motors, shows a gap in the literature.

The main challenge of building electric motors with current manufacturing systems is the limited available materials that can be processed using 3D printing machines. For instance, an electrical conductor is one of the essential elements in an electric motor. However, the lack of mature AM technologies able to produce a high electrical conductivity material such as copper is considered as a challenge. A conventional DC motor is also constructed of permanent magnets (PMs), and the most powerful PM's should have an organized granular structure that is a challenge to rebuild with a 3D printing machine. Furthermore, 3D printing the rotor and stator cores with high permeability soft magnetic materials that determine the motor performance are still under development.

Based on the above principles and a detailed literature review, the brushed DC electric motor (BDCM) is selected to be entirely $3 \mathrm{D}$ printed. The selection of the BDC motor was due to its construction from fewer parts than other motor types. The electrical power source in a classical BDC motor is connected to the rotor winding through a commutator and brushes without the need for the electrical power source electronic commutation based on hall 
position sensors. Besides, all other motor types are derived from its design. The selection of the AM technique in this research revolved around considering several issues, including the availability, materials, costs, process of manufacturing, and the state of the art of the AM technologies available. For comparative purposes, The three rotors are produced considering different additively manufactured materials using Fused Deposition Modeling (FDM) and Cold Spray Additive Manufacturing (CSAM). The chosen materials were to study the behaviour of the printed silicon steel with the same percentage used in conventional motors and the Proto-pasta Magnetic Iron Metal PLA Composite behaviour when used as a softcore.

Several prototypes of Self-excited DC motor were 3D printed in order to eliminate the use of permanent magnets in the stator using different materials and different printing techniques, followed by the building process of pancake motor in order to eliminate the use of the copper coils. Optimization techniques are not addressed in this project since the first goal is to 3D print electric motor not to improve its performance. Also, the building of the axial flux DC motor (Pancake Motor) was manufactured only to develop the coils technique to eliminate the copper coil winding.

\subsection{Research Scope, Aim, Novelty, and Objectives}

\subsubsection{Research Scope}

The scope of the present research is defined based on the study carried out in this reaserch. Silicon steel (Fe-Si) alloy is selected as the raw material of choice to create the soft magnetic core. The selection was based on its high electrical resistivity, which helps to reduce the eddy currents, excellent magnetic properties and good raw material availability.

As regards the $3 \mathrm{D}$ printing technology used for processing the raw material of the core, Cold Spray Additive Manufacturing (CSAM) is preferred for important technical reasons. CSAM can produce metals with different melting points, which makes it capable of depositing silicon steel alloy with desirable compositions. Researchers also are examining the use of laser powder bed fusion (LPBF) with soft magnetic materials such as silicon steel [3], [4], [5] and hard magnetic materials such as neodymium-based materials [6], [7].

Fused Deposition Modeling (FDM) is also considered for creating the soft magnetic core, electrical insulation, and for exploring the use of copper PLA to create the conductive winding. Laminated Object Manufacturing (LOM) is also preferred to explore the possibility of creating the copper winding traces using copper foil. 3D printed permanent magnets are also considered as an option to be provided by a third party, owing to the probability of the 3D printing technology for processing the hard magnets. Optimization techniques are not discussed in this research as the first intention is to $3 \mathrm{D}$ print a DC electric motor. While benchmarking and analyzing the final fully 
3D printed DC motor will be performed.

\subsubsection{Research Aim}

The present research work aims to adopt 3D printing techniques to demonstrate the feasibility of producing printed electric motors by implementing several separate AM machines. All parts of the motor will be produced using just AM methods to create a functional electric motor. Benchmarking the 3D printed motor experimentally against representative commercial-off-the-shelf motor to validate its electromechanical performance is also proposed as part of this research work. Achieving the goals mentioned above will, in turn, allow demonstrating the applicability and benefits of 3-D printing technology in the manufacturing development of electric motors.

\subsubsection{Research Novelty}

To the author's knowledge, the novelty of the proposed research, which is the fabrication of fully $3 \mathrm{D}$ printed electric motors through AM technology, has never been attempted in the literature.

\subsubsection{Research Objectives}

The objectives of this research are outlined in the following points:

\section{Investigate the possibility of printing the rotor core of PM DC motor using silicon steel with different composition via CSAM and magnetic iron PLA via FDM:}

(a) Wind each 3D printed rotor with the same conductive material (copper), and the same number of turns then assemble with conventional stator constructed of PMs.

(b) Test the performance of the printed cores with respect to the torque and angular velocity.

\section{Investigate the possibility of eliminating the PM from the stator through constructing Self-excited DC motor:}

(a) Replace the PMs with a field coil winding on two 3D-printed pole shoes constructed by AM technologies using different materials considering properties describe good core soft magnets for motor applications.

(b) Test and evaluate the 3D-printed pole shoes with respect to the produced electromagnetic force. 
(c) Assess how both the design strategy and material properties affect the produced electromagnetic force.

\section{Investigate the possibility of eliminating the copper coil winding of the rotor through constructing a printed circuit motor and the option of using conductive copper filament or any other AM techniques:}

(a) Replace the copper winding technique on the rotor with a printed circuit board rotor.

(b) Swap the printed circuit board rotor with a fully 3D printed rotor.

(c) Test and evaluate the printed rotors with respect to the produced electromagnetic force.

(d) Assess how the design strategy of number of windings, rotor size, and winding shape affect the produced electromagnetic force.

\section{Manufacturing of the final motor design and benchmarking to compare performance with an off-the-} shelf motor:

(a) Once the above list of objectives is fulfilled, the final task is to fabricate the fully 3D printed motor using multi-techniques and multi-materials.

(b) The prototype shall be employed to validate the electromechanical performance experimentally to compare with the classically manufactured counterparts directly.

The thesis structure is laid out according to the following; In Chapter 2, the literature review is presented, intended at providing guidelines towards the selection of electric motor type, proper materials and compatible AM techniques to build each part of the motor. In Chapter 3, the experimental methods applied to fabricating and testing of radial-flux DC motor are described. In Chapter 4, the practical techniques used to manufacture and test axial-flux DC motor are reported. Chapter 5 provides a direct comparison with the classically manufactured counterparts to validate the motor's electromechanical performance experimentally. In Chapter 6, the conclusion and future work are represented. 


\section{Chapter 2}

\section{Literature Review}

\subsection{History and Application of Additive Manufacturing}

\subsubsection{Introduction}

The possibility of electric motor manufacture utilizing $3 D$ printing techniques is presented in this chapter. This chapter begins with an overview of the leading Additive Manufacturing $(A M)$ technologies available on the market, followed by an insight into the electric motors technology and main motor types. Then, the active parts of a typical electrical machine, such as the soft magnetic cores and the permanent magnets that form the motor rotor and stator, were described with regard to the appropriate materials.

The study allows us to conclude that the conventional Brushed $D C$ Motor $(B D C M)$ has the highest potential for manufacturing using $A M$ technologies due to its simplicity, as well as the fact that all other motors are derived from its design. Some techniques represent the best option to achieve a particular part of the motor structure, such as the soft magnetic core that can be produced using Laser Powder Bed Fusion ( $L P B F)$. In contrast, other parts of the motor could be challenges on how to be manufactured using AM machines, such as the electrical conductors. Although AM machines now can print copper, fabricating 3D-printed copper conductors that work as ordinary copper wires is not yet achieved due to the lack of mature AM technologies to produce a high electrical conductivity material in the form of a coil. Therefore the investigation for an alternative method is addressed in this project. An overview of motor winding made of an electrically conductive material and the commutator is also discussed in this thesis and considered essential components of electric motors.

\subsubsection{Additive Manufacturing Technologies}

Virtually any geometric form or model can be constructed using $A M$ techniques. The term $3 D-$ printing 
was initially used to describe a process that uses printer heads to deposit a binder material over a powder bed, layer by layer [8]. More recently, ' $3 D$-printing' indicates various types of $A M$ techniques; $A M$ is more popular due to its broader meaning and length of usage. Rapid prototyping $(R P)$, desktop manufacturing $(D T M)$, rapid manufacturing and direct digital manufacturing are other commonly used terms. Early $A M$ devices and materials were developed in the 1980s [9]. In 1981, Hideo Kodama of the Nagoya Municipal Industrial Research Institute published a report on a functional $R P$ system that uses photopolymers to build a solid, printed model in layers, each corresponding to a cross-sectional slice in the model [10].

In 1984 SLA US Patent 4575330, filed by Dr. Chuck Hull in 1984, marked the birth of the additive manufacturing industry. Dr. Hull coined the term "stereolithography" (SLA) and in his patent on "apparatus for production of three-dimensional objects by stereolithography”. 1987 SLA, the first SLA product, emerged from 3D Systems, a process that solidifies thin layers of ultraviolet (UV) light-sensitive liquid polymer using a laser [11].

At the same time, a company called DTM Inc. created the world's first Selective Laser Sintering $(S L S)$ device, which focuses a laser on powder rather than liquid [12]. As these technologies were in the early stages, the equipment was costly for individuals, and there were defects in the produced parts. However, they did demonstrate significant potential [13].

From 1999 to 2010, the first 3D-printed organs were implanted in humans. Scientists at the Wake Forest Institute for Regenerative Medicine 3D-printed the structure of a human bladder, then coated it with the patient's cells. The newly created tissue was then implanted into the patient, and there was a low probability that the immune system would reject the tissue because it was their own cells. This achievement was a significant milestone in $3 D$-printing [14].

Over the past ten short years, scientists from different institutions have developed a functional miniature kidney [15] and built a complicated prosthetic leg [16]. The bioprinting company Organovo created the first $3 D$-printed blood vessels using only human cells [17].

In 2005, Dr. Adrian Bowyer launched the RepRap Project, an open-source 3D-printing system that can selfreplicate by printing most of its parts [18]. This gave virtually everyone the ability to create almost anything they could envision [19].

In 2006, The first $S L S$ [20] 3D-printers became commercially viable in 2006, and this was the real beginning of on-demand manufacturing of industrial parts. Also, in 2006, a 3D-printing startup company Object (now merged with Stratasys) developed a 3D-printer that could print with multiple materials. This allowed a single part to be built in various forms, with different material properties [21].

The inspired innovations of the 2000/10 decade ended with the launch of collaborative co-creation services. One of them is Shapeways [22], a 3D-printing marketplace where designers can solicit feedback from consumers 
and other designers, and fabricate their products relatively inexpensively [19]. Another service, MakerBot, provided an open-source DIY kit for makers to build 3D-printers and products [23].

In 2011, aerospace engineers at the University of Southampton designed, built and flew the world's first 'printed' aircraft. The entire structure of the Southampton University Laser Sintered Aircraft (SULSA), from the wings to the integral control surfaces, was printed [24].

In 2013, the KOR Ecologic project completed the first $3 D$-printed car called URBEE, a car with a $3 D$-printed body built to get $200 \mathrm{mpg}$ on a freeway. It was the first car to have a $3 D$-printed body and is still the greenest practical car ever made [25].

In 2014, the Local Motors Strati $3 D$ car was printed live at the International Manufacturing Technology Show (IMTS). The process took 44 hours [26].

$N A S A$ announces a plan to explore the possibility of $3 D$ printing food in space for astronauts [27], and in 2014 a printer was implemented at the International Space Station $(I S S)$, which successfully printed the first part ever manufactured in space. This will allow astronauts to print replacement tools directly in space, thereby reducing the need to bring additional supplies [28]. NASA also successfully tested the first $3 D$-printed rocket fuel injector, and it exceeded those made of traditional materials [29].

In 2015, Foster and Partners and several smaller firms teamed up with the European Space Agency (ESA) to develop a lunar dwelling, using $3 D$-printed parts transported by rockets and $3 D$-printed materials using the moon's regolith. The structures were designed for the moon's South Pole, to protect inhabitants from meteorites, gamma radiation and temperature variations [30].

The fundamental intention of the expanded field of $3 D$-printing is the capability for printers to either replicate themselves or create many of the critical components needed to build a machine. Interestingly, many consumer $3 D$-printers are now made up of parts printed in $3 D$ [31]. As technology advances and the industry develops, other important machine components will be $3 D$-printed. In the near future, we will able to $3 D$-print all the components necessary to produce complex structures, such as houses, cars and machinery [32].

The possibilities of $3 D$-printing are fundamentally unlimited for many industries, including plastics, metals, food and organic materials, and it can employ a wide range of materials. $A M$ offers a different approach when compared to subtractive or forming methods, and for this purpose, it represents many benefits. Significantly, $A M$ increases the design freedom greatly, as a result offering exciting possibilities when products with new functional geometries can be produced at less or no added cost. It has been demonstrated that AM helps lower the manufacturing costs for parts that would be extremely costly if manufactured with conventional processes [33]. Currently, $3 D$-printers only manufacture products out of resin, plastic, ceramics and certain metals. The potential to $3 D$-print products with mixed materials and different technologies, such as circuit boards and electric motors, is under de- 
velopment. Because AM technologies add material only where required, no production line is required to make specialized tools such as fixtures, jigs, dies and moulds that are needed in the traditional process. This advantage makes $A M$ very useful for small quantity production where a part's geometrical design is more critical than its per-unit cost. In this regard, the aerospace industry has been showing a growing interest in the $A M$ technologies for the fabrication of end-use parts and assemblies [1]. One of the difficulties of $A M$ technology implementation is low productivity compared to traditional manufacturing [34]. Other difficulties to $3 D$ printing implementation are the high machine and raw materials prices, and the limited available materials that can be processed using 3D printing machines [35]. The quality control of the AM final products is also considered an issue that has yet to be examined. For AM products to be used as final products, they should be passed through an advanced quality control process to reach the desired reliability and replicability [36]. Many different 3D-printing processes use a variety of materials available today, including plastics, metals and waxes. The most popular 3D-printing processes are $F D M$, stereo-lithography apparatus $(S L A)$ and selective laser sintering $(S L S)$. Though there are many different printing processes, they all form $3 D$ objects using solid, liquid or powder-based materials. Thus, all $3 D$-printing techniques follow the same basic process [37].

This basic $3 D$-printing process involves the following steps:

1. Creation of the digital model of the design with any CAD (computer-aided design) software such as PTC Creo, Pro/Engineer or Auto-Cad.

2. Conversion of the CAD model into the Stereolithography file Format $(S T L)$. The STL file format then represents all surfaces in preparation for the slicing algorithms.

3. Slice the converted $S T L$ file into 2D cross-sectional layers. The third step includes slicing the $S T L$ file using a specific software program provided by the $3 D$-printing machine manufacturer. The slicing software imports the $S T L$ file, which allows the user to adjust the size, orient the part and determine the slice thickness of the model, which can vary from 0.01 to $0.7 \mathrm{~mm}$ depending on the capabilities of the $3 D$-printer. Though thinner slices improve the accuracy of the prototype, they also increase the time to build the part. The pre-processing (slicing) software generates a thin-walled structure to support internal cavities and overhang sections of the model during the buildup. The software also determines how much time and length of filament required to make the prototype. The following step is then deciding on the different parameters of your part and the printing process. This process involves deciding on the size and placement of your print on the platform.

4. 3D-print the prototype. The fourth step is to create the prototype. The slicing software will convert the 
data from the STL file into a Gcode, a specific code containing instructions for the printer. Then it is sent to the $3 \mathrm{D}$ printing machine, which begins to build up the prototype one layer at a time.

5. Remove and clean the model. The fifth and final step is to remove the printed part from the machine and clean it to improve the appearance, surface finish and durability; this could require painting, sealing or sanding [38]. Depending on the AM process and material system, there may be additional post-processing steps after printing, such as support structure removal, machining, heat treatments, UV curing, etc. [39]

Among the different technology facilitators for modern electrical machines, additive manufacturing plays a key role. The advantage of having precise control of the shape of ferromagnetic structures while accomplishing good electromagnetic performance goes well with the design requirements of rotating electrical machines. To a specific degree, some of the material's physical properties may be allowing for quick trade-off studies using prototyping instead of conventional manufacturing techniques, despite being considered an enabling technology. AM of soft magnetic materials for electric motors is yet at an early stage [40]. This project, therefore, aims to provide initial proof of concept. For this purpose, a brushed DC electric motor (BDCM) is selected to be entirely 3D printed. Its rotor core is additively manufactured through Fused Deposition Modeling (FDM) and Cold Spray Additive Manufacturing (CSAM). Its performances are analyzed to those of an identical commercial motor through indepth experimental tests. Preliminary results show that the 3D printed machine can develop the rated power but with an efficiency reduction.

\subsubsection{Tradeoffs between AM Processes}

There is a series of decisions to optimize for one or more essential characteristics needed in the final object, such as type of materials, strength, speed of printing, minimum feature resolution, and cost. 3D printing optimization requires an understanding of the tradeoffs that different processes and parameter settings offer. Highlight the tradeoffs between the processes of the leading 3D printing technologies commercially available for manufacturing end-use parts is presented in Table 2.1 [41], [42]. The research aim is to develop techniques for 3D print electric motors. In the meantime, the electric motor is usually made of three types of materials: insolation, high permeability alloy, and conductive materials. Therefore, the 3D printing technologies of interest for this work are: 
1. Fusion Deposition Modeling (FDM) for polymer-based motor parts as insulation material and magnetic iron metal PLA Composite as a soft magnetic core.

2. Laser Powder Bed Fusion (LPBF) and Cold Spray Additive Manufacturing (CSAM) to form the soft magnetic cores produced from metal alloys.

3. Laminated Object Manufacturing (LOM) to create conductive material. 
Table 2.1: Tradeoffs between the processes of the leading 3D printing technologies commercially available, Source: Adapted from [41], [42],[43], [44].

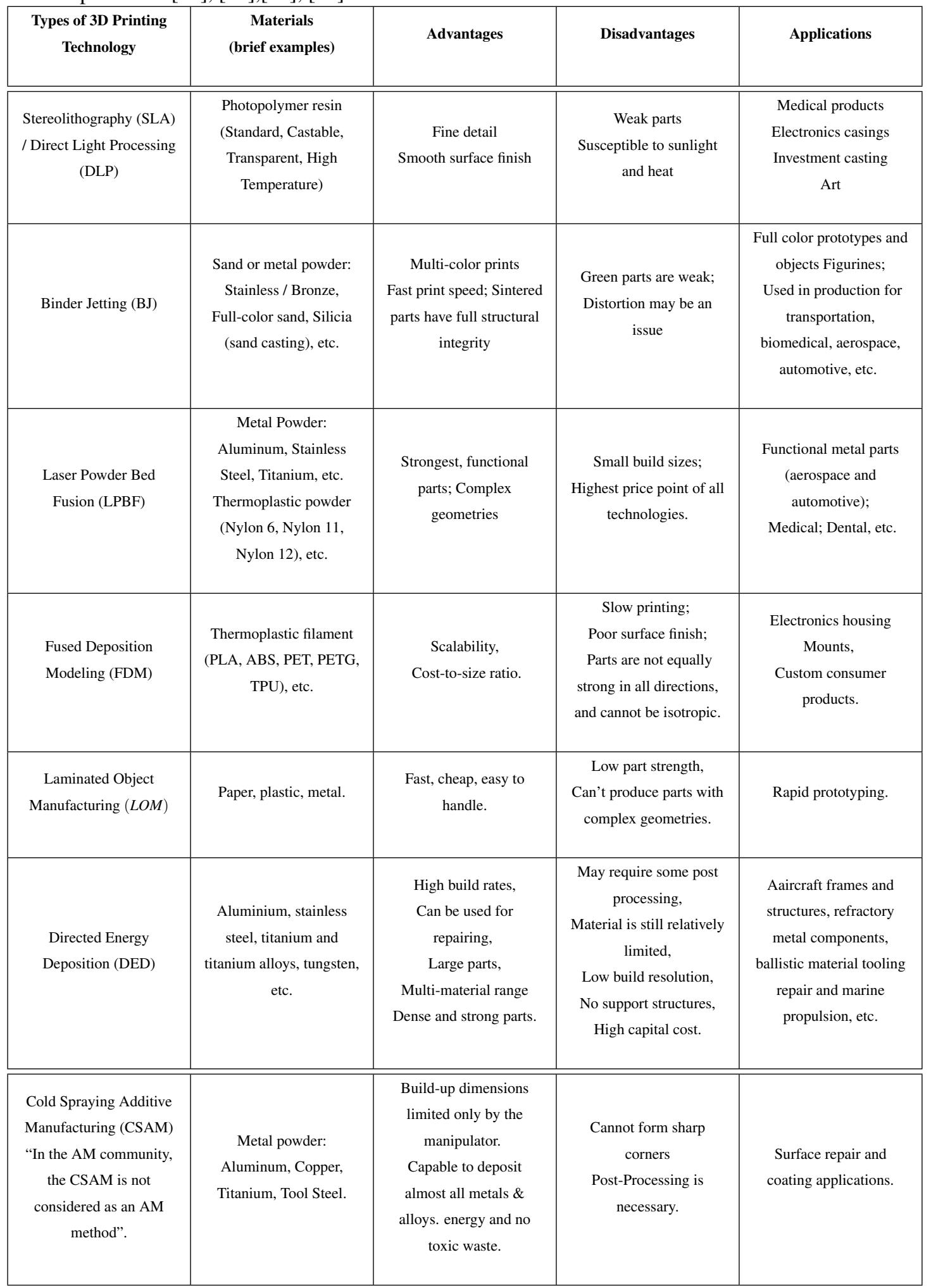


The technical aspects of all studied technologies are described in the following sections. On the other hand, It was noted that the only 3D printing capable of processing high-density metals and alloys with an appropriately excellent surface finish are: Electron Beam Melting (EBM) and Laser-Powder Bed Fusion (LPBF). These processes could be used to establish soft magnets motor parts similar to the conventionally manufactured. Yet, the high cost of these techniques and the limited accessibility makes them hard to use in our prototypes where the selestion of AM in this project was dictated by availability. They might be used in the future to increase the fully printed motor's performance once the final processes are identified using low-cost technologies. In contrast, the Stereolithography (SLA) technique was excluded due to the weakness of its constructed parts. The Binder Jetting (BJ) technique and Directed energy deposition (DED) were also excluded because the availability dictated our AM selection.

\subsubsection{Fused Deposition Modeling ( $F D M)$}

Fused Deposition Modeling (FDM) is known as Material extrusion and is one of the commonly used applications of 3D-printing [45]. Replicating Rapid Prototyper machine (RepRap) [46]shown in Figure 2.1 is one of the largest open-source development communities that use this type of 3D-printing technology, and its development has led to significant cost reductions. However, this technique is highly anisotropic and has dimensional accuracy limitations [47]. Material extrusion 3D-printing uses a thermoplastic material filament as a base material. The filament is fed to a heated moving extruder, and the molten material is pushed out of the extruder nozzle and deposited on a $3 \mathrm{D}$ printing bed. The printer platform can also be heated for additional adhesion according to manufacturer specifications. After the first layer is complete, the second layer is deposited on top of it, and the process is repeated until the designed part is finished. The computer controls all movements of both the extruder head and the printer platform [48]. A wide variety of filament materials are compatible with this type of printing, the most popular being; Acrylonitrile Butadiene Styrene (ABS), Polylactic Acid (PLA), High-Impact Polystyrene (HIPS), Thermoplastic PolyUrethane (TPU), and Aliphatic PolyAmides (PA).

Recently, the material extrusion technique has been applied for 3D-printing of composites. The base material is thermoplastic, and there must be enough material to ensure fusion between the layers. Mixing two elements in a single filament makes it possible to produce magnetic iron PLA, wood PLA and copper PLA by embedding iron, wood and conductive copper particles in the PLA to form the final products. There are a variety of other metal infused filament options, for multiple material systems. [49], [42], [50]. The FDM technique was one of the $3 \mathrm{D}$ printing technologies of interest for this work. In this work, the FDM technique was used to form the polymer-based motor parts as insulation material and magnetic iron metal PLA Composite as a soft magnetic 


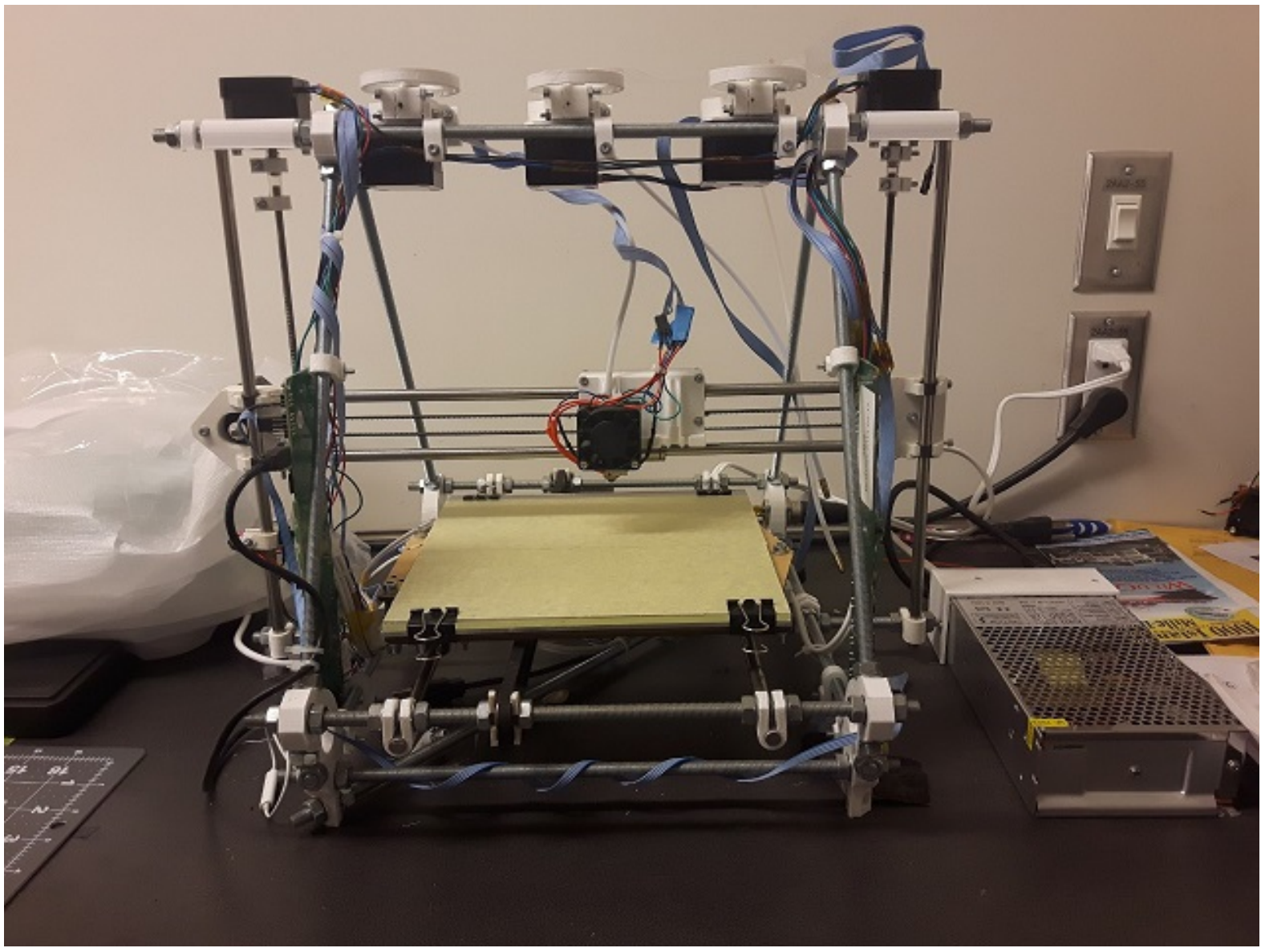

Figure 2.1: Fused Deposition Modeling (FDM) RepRap 3D Printer (Carleton University)

\subsubsection{Laminated Object Manufacturing $(L O M)$}

In 1984 Kunieda created the concept of LOM [51], and later in 1986, it was developed by a company called Helisys [52]. In 1991 the company commercialized the LOM machine, which made 3D products with paper rolls and a laser. The raw material used in LOM was copier paper, coated on one side with thermally activated adhesive. The bonding and lamination of stacking layers are done using a heated roller. When a heated roller passes over the stacked sheets, the coating melts and bonding between the sheet and substrate. The building stack is cut by a laser beam or a knife to provide the required 3D model. This process is followed by the lowering of the platform by a distance equal to deposited layer thickness. The extra material which does not form the model is then removed. This procedure is repeated until the final part is then obtained [53]. The LOM technique is schematically introduced as illustrated in Figure 2.2 


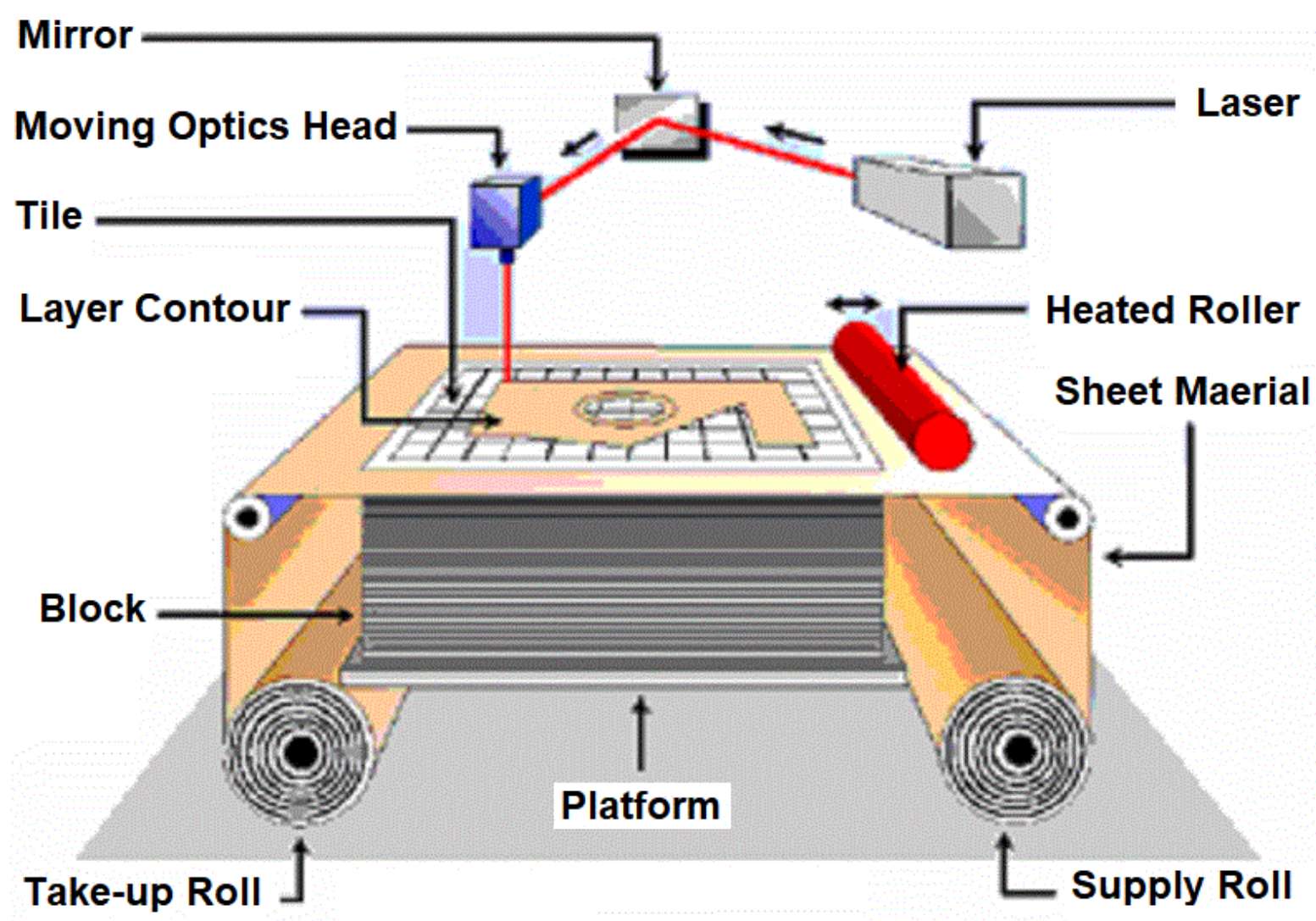

Figure 2.2: Laminated Object Manufacturing (LOM), Source: [54]

Rolled thin sheets of different materials such as plastics, paper, ceramics, metals, cellulose, polycarbonate composites, etc. are used as materials for the LOM technique. The LOM offers some advantages over other AM techniques such as; no support structure is required, high production speed, inexpensive raw material, reasonable set-up cost, low thermal stresses, low distortion and deformation during processing, no chemical reaction involved, and significant components can be produced. In contrast, The main downsides of LOM include; weak interfacial bonding among layers, only suitable for laminated sheets, poor surface finish, poor dimensional accuracy, and difficulty in producing hollow parts. In this project, the LOM-like technique was applied to create conductive material using simple tools. The implementation of such a process was caused by a lack of access to LOM technology.

\subsubsection{Laser Powder Bed Fusion (LPBF)}

Both Direct Metal Laser Sintering (DMLS) and Selective Laser Melting (SLM) is a metal 3D printing method that belongs to the powder bed fusion additive manufacturing family. This technology uses a laser to scan and selectively melt the metal powder particles, bonding them together and constructing a part layer-by-layer. Both 
technologies produce objects in a similar fashion to SLS. The main difference is that these 3D printing technologies are applied to the production of metal parts. SLS does not melt the powder but instead heats it to the point that it can fuse on a molecular level. DMLS and SLM use the laser to melt the metal powder forming a homogeneous part. DMLS and SLM refer to the same tech, and there is no difference in printing alloys versus pure metals in terms of the process or machines used. Unlike SLS, the DMLS and SLM processes require structural support to avoid distortion and enable heat transfer away from the process zone to prevent vaporization. DMLS/SLM parts are at risk of warping due to the residual stresses produced during printing because of the high temperatures. Parts are also typically heat-treated after printing while still attached to the build plate to relieve any stresses in the parts after printing [55], [56], [42], [50], [57].

The critical elements of this process consist of a (1) laser system, (2) powder delivery system, (3) recoater blade, and (4) build bed and feed bed. Firstly, the powder delivery piston-driven chamber travels up, and the fabrication piston-driven chamber travels down by the distance of one layer thickness. Next, a recoater blade spreads the powder above the surface of the fabrication piston. Some systems use a hopper mechanism to deliver the powder instead of a powder feed bed. A laser beam is then focused over the bed in order to melt the powder under the control of a scanner system. Once completed, the fabrication piston moves down another layer thickness, and a new layer of powder is spread over it. The procedure is repeated until the whole part is fabricated. The extra powder is cleared away and can be reused after proper treatment [58], [56], [59]. An overview of the LPBF technique is provided as illustrated in Figure 2.3. 


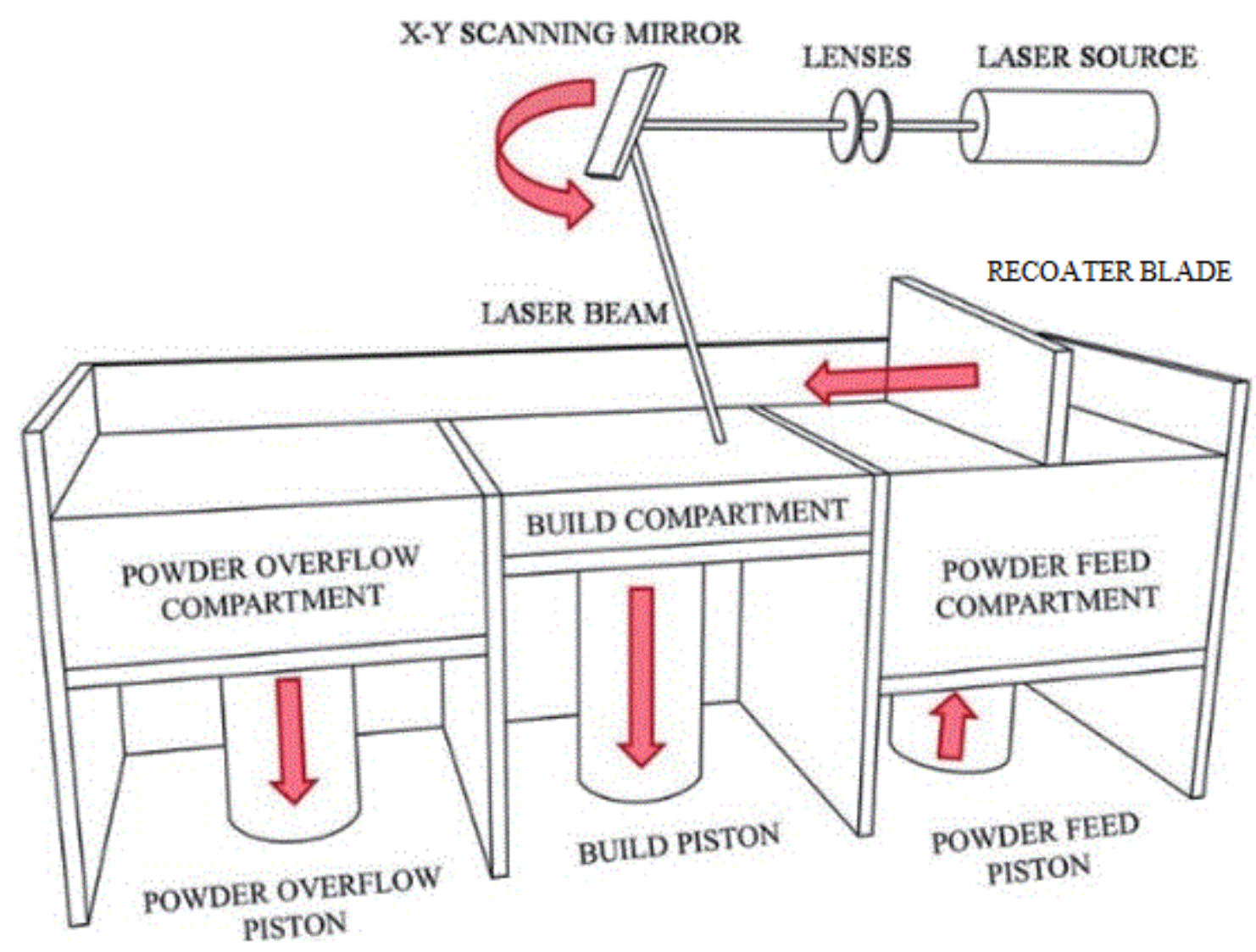

Figure 2.3: Schematic of LPBF, Source: [60]

There are many advantages of the LPBF technique. Firstly, its applicability to process a wide range of metallic alloys, including tool steels, titanium, nickel-based superalloys, and stainless steels, which are commonly available in powder form. In addition to its ability to produce complicated geometries, furthermore its high accuracy in exquisite detail. At the same time, there are many disadvantages of the LPBF technique, including the slow process compared to other technologies, support structures are mandatory, relatively small build size, high power required, extensive cleanup is essential due to a large amount of unused powder and the produced parts usually have a rough, grainy surface finish and need post-processing (surface finish depends on powder size). In this work, the LPBF technique was used in this work to form the soft magnetic cores produced from metal alloys.

\subsubsection{Cold Spraying Additive Manufacturing (CSAM)}

The idea of Cold Spraying Additive Manufacturing (CSAM) was invented in the 1980s. Papyrin and others [61] initially demonstrated CSAM at the Russian Academy of Sciences, Novosibirsk. They employed CSAM for the deposition of a variety of metals and composites on different types of substrates. The applicability of CSAM 
during the last two decades has been increasing. Industrial applications of the CSAM technique include the coating of pure metal and alloys, functional coatings, composites for better corrosion, wear, and high-temperature resistance. CSAM is used for all types of pure metals as well as alloys. In Addition to coating applications, CSAM is utilized as an appropriate technique for the repair of failed parts [62], [63].

Although CSAM is not considered an AM method in the AM community, NRC published a paper that overviews the process, describing the basic mechanisms of consolidation and describes the technologies associated with future positioning of this technology in additive manufacturing. The authors say that the main similarity with more conventionally recognized AM technologies is that cold spray is fundamentally a powder deposition technique for additive manufacturing. As a coating technology, cold spray's potential in additive machining instead of subtractive machining is currently well recognized and developed. Compared to additive manufacturing techniques, the main advantage is that component size is unlimited; the main disadvantage is that cold spray does not have a comparable areal resolution of features, even with new developments in micro nozzles. On the other hand, layerby-layer build strategies can be implemented, increasing the capacity to accomplish more complex builds by cold spray. The authors concluded that the key to implementing cold spray additive manufacturing is hybridization, whether with laser-based technologies or conventional subtractive machining, to maximize both additive and subtractive machining strengths [64]. Accordingly, NRC provided us with the silicon steel to build the motor rotor discussed in this project, considering this technology belongs to AM technologies.

The CSAM technique uses a supersonic jet of high temperature compressed gas (generally N2 or He gas stream) to accelerate the powder particles for their deposition over a substrate. The temperature of the expanded gas stream remains quite low $\left(100-500^{\circ} \mathrm{C}\right)$, and usually is less than the powder melting point temperature, that is why it is known as a cold spray. The size range of material particles is between $10-100 \mu \mathrm{m}$ in diameter. The machine set-up mainly uses a powder feeding system, a compressed gas, a heating system to heat the compressed gas, a supersonic nozzle, a robotic arm, as well as other functional arrangements [65]. A schematic arrangement of a CSAM technique is illustrated in Figure 2.4. In its most straightforward operation, high temperature compressed gas expands in the nozzle to create a supersonic flow. The powder particles (metals or composites) are exposed to the nozzle inlet and then attain a high velocity by a supersonic jet; they are then guided in the direction of a substrate forming a bond with the surface due to impact. This procedure is repeated to increase the thickness of the deposit. Machining is needed after the completion of deposit thickness to achieve desired dimensions and shape [62], [63]. 


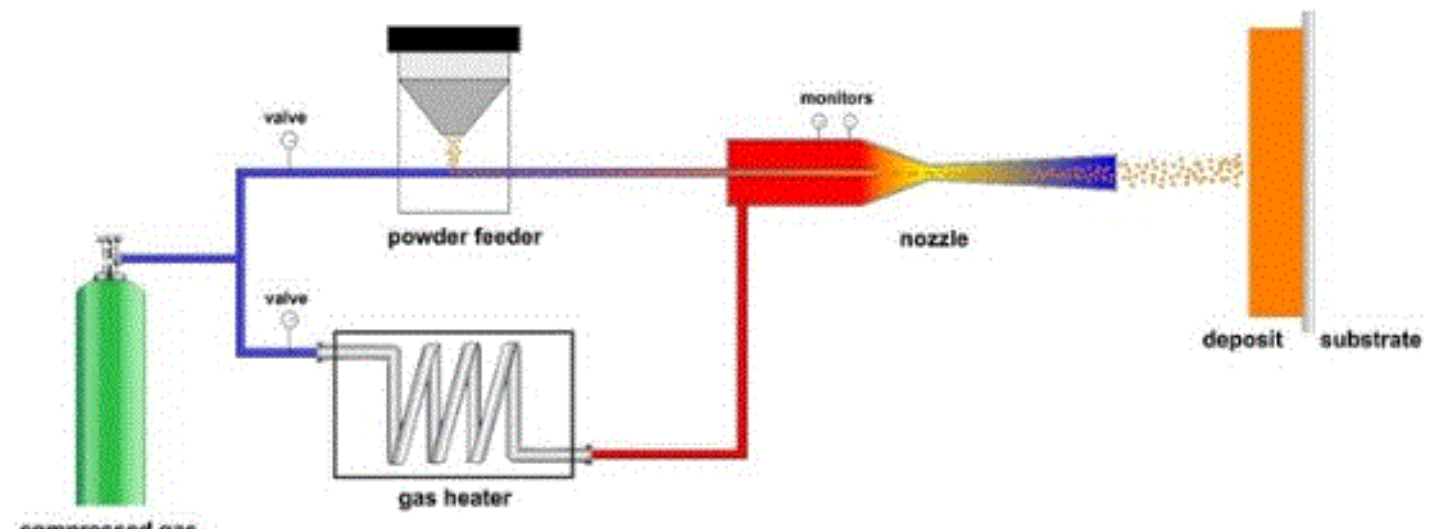

(a) high pressure cold spray system

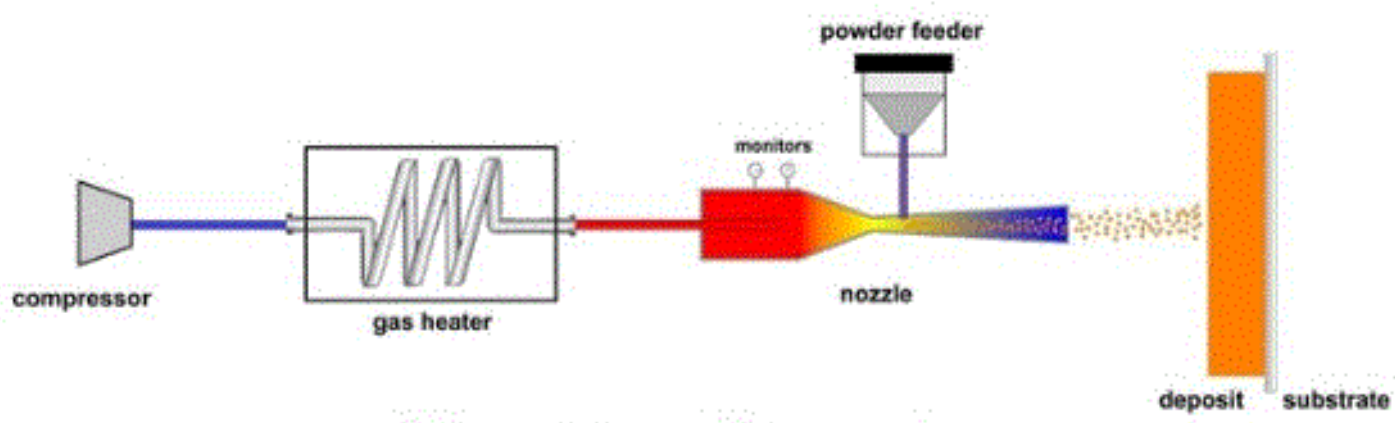

(b) low pressure cold spray system

Figure 2.4: Schematic Arrangement of High and Low Pressure CS Systems, Source: [66]

As compared to conventional fusion-based AM techniques, CSAM has unique benefits, including works in solid-state processing during its layer-by-layer additive manufacturing, shorter fabrication time with superior flexibility, appropriate for fixing damaged parts, and production of high reflectivity metals, unrestricted production volume, and suitable for temperature-sensitive substrates and for creating composite components.[66], [67], [43]. However, the products made using CSAM need post-machining; This is because CSAM deposits have rough surfaces that require post-heat treatment to accomplish better mechanical properties [43]. The application of CSAM can be observed in two ways; First, repairing of engine blocks of cast iron, aircraft components, worn surfaces. Second, manufacture of parts with rotational symmetry such as flanges, cylinder walls. In this work, the CSAM technique was used in this project to form the soft magnetic cores made from metal alloys. 


\subsection{Elements of Electric Motor Construction and Design}

\subsubsection{Electric Motor Construction}

Electric motors take electrical energy and produce mechanical energy. Motors come in different sizes. Examples of small electric motor applications include motors used in robots, automobiles, hand power tools and food blenders. Examples of sizable electric motor applications include metal rolling mills, elevators, and electric trains. Electric motors are categorized into two different categories, Direct Current (DC) and Alternating Current (AC). There are many types within these categories, each offering unique abilities that suit them well for specific applications.

An electric motor consists of two parts, the stator and the rotor, separated by an air gap. Typically, the rotor is the rotating section and carries the winding, while the stator is stationary and holds the permanent magnets (PMs). The rotor is typically made of silicon steel, which is available in thin lamination sheets and cut by laser to form the desired patterns. The sheets are connected to form the final shape of the rotor cores [68]. In this study, the brushed DC motor (BDCM) was chosen to be studied and 3D-printed. The electric power source in a classical BDC motor is connected to the rotor winding through a commutator and brushes. There is no need for electronic commutation based on hall position sensors [68]. On the other hand, brushless DC motors, linear motors, and AC motors are not considered in this project because they do not use brushes. In comparison to brushed DC motors, these motors use a very different method of commutation. Instead of brushes, commutation is done electronically [69]. For simplicity, the motors that need controller devices such as current control, feedback systems and Pulse Width Modulation (PWM) are not examined in this research. The exploded view of DC electric motor as illustrated in Figure 2.5 shows the functional components and materials of a traditional permanent magnets DC brushed motor. In the armature part or rotor, a rotating magnetic field is generated using an array of electromagnets. The magnetic field produced in the rotor interacts with the static part, namely the stator, resulting in rotational speed and torque production. There are different types of D.C. motors, but they all work on the same principles. The specific electromagnetic nature of the interaction between the rotor's magnetic field and the stator determines the topology of the motor [70], [71]. 


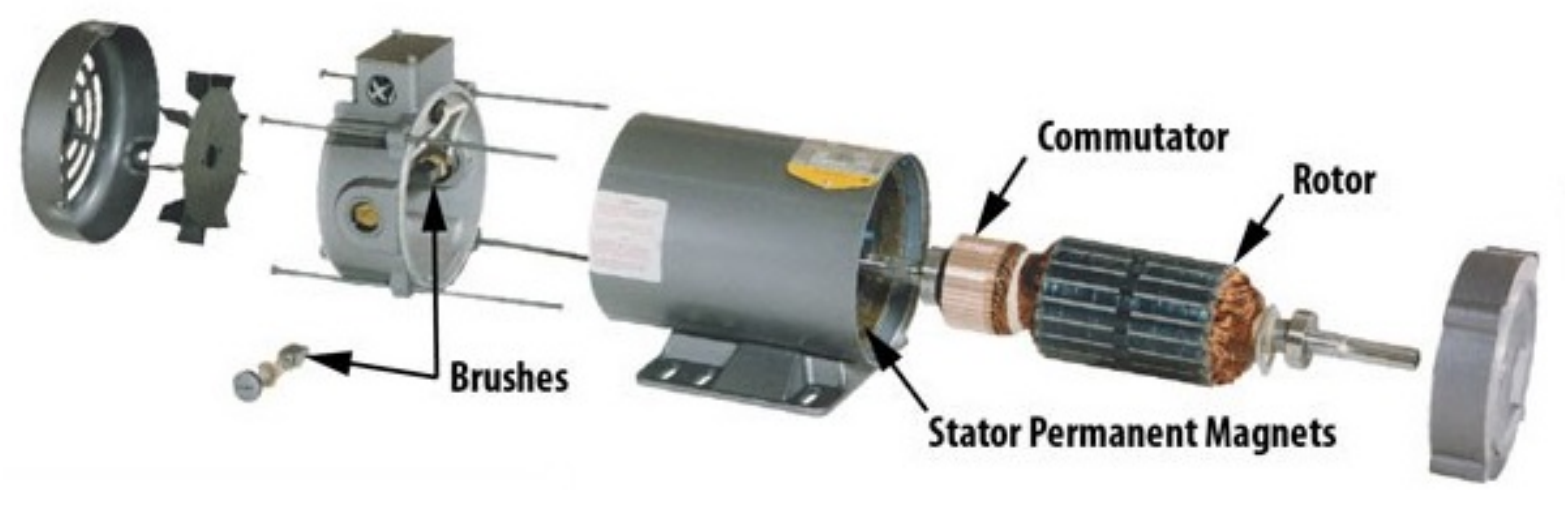

Figure 2.5: DC brushed motor Exploded view, Source: [72]

Since the development of the DC motor in the first half of the 1800s, many machine topologies have been launched. The advantages and drawbacks linked with the different machine types make them suitable in different application fields, power ranges and different operating conditions. In this literature, the radial and the axial flux BDC motors present the highest potential. In the Permanent Magnet (PM) motor, a hard magnetic material such as rare-earth materials, aluminum-nickel-cobalt compounds or hard ferrites is situated on the stator core. In this case, a torque is generated between the opposite poles of the rotor field and the permanent magnets. An example of a standard PM motor design is illustrated in Figure 2.6(a). The rotor is only composed of a soft magnetic core and represents the most straightforward and cheapest rotor form. Therefore, motor performance relies on the quality of the soft magnetic materials used, as well as on the geometrical design of the rotor and stator cores [73].

In self-excited shunt DC motors, the interaction depends on the electrical current induced inside several electrically conductive copper windings arranged within the rotor core by the rotating magnetic field. The induced current generates a magnetic field that attracted or repealed the stator produced magnetic field, consequently creating mechanical work. An example of a shunt DC motor design is illustrated in Figure 2.6(b). 


\section{Permanent}

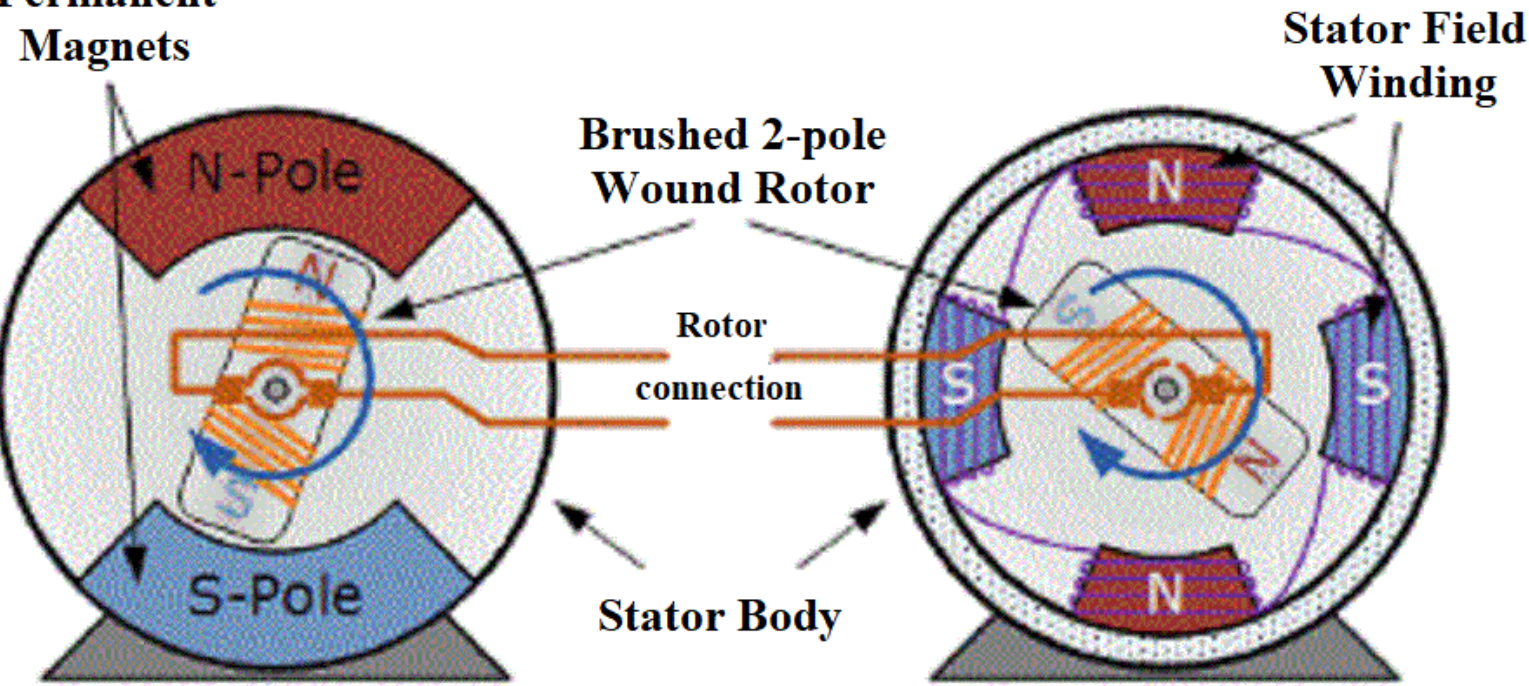

(a) 2-Pole Permanent Magnet Motor

(b) 4-Pole Wound Field Motor

Figure 2.6: (a) Standard PM Motor Design (b) Shunt DC Motor Design, Source: [74]

\subsubsection{Working Principles of Brushed Permanent DC Motor}

A DC motor has two input terminals, and a voltage applied across these terminals causes the motor shaft to spin. For a constant load or resistance at the motor shaft, the motor shaft achieves a speed proportional to the input voltage. The positive voltage causes spinning in one direction, and negative voltage causes spinning in the other[75].

\subsubsection{Motor Physics}

DC motors exploit the Lorentz force law [76],

$$
F=l I \times B
$$


Where: $F, I$ and $B$ are three-vectors,

$F=$ The force on the conductor

$l=$ The length of the conductor (in the magnetic field)

$I=$ The current vector (including the magnitude and direction of the current flow through the conductor)

$B=$ The magnetic field created by permanent magnets

Since we have a loop of wire rotated about its center as illustrated in Figures 2.7 and 2.8. In one half of the loop, the current flows into the page, and in the other half of the loop, the current flows out of the page. These opposite forces create torque on the loop about its center at most angles $\theta$ of the loop.

The total torque acting on the loop around its center can be expressed as

$$
\tau=2 d f \cos \theta
$$

Where:

$d=$ The distance from the halves of the loop to the center of the loop

$f=$ The magnitude of the force acting on each half of the loop.

$\theta=$ The angle of the loop with respect to the magnetic field B

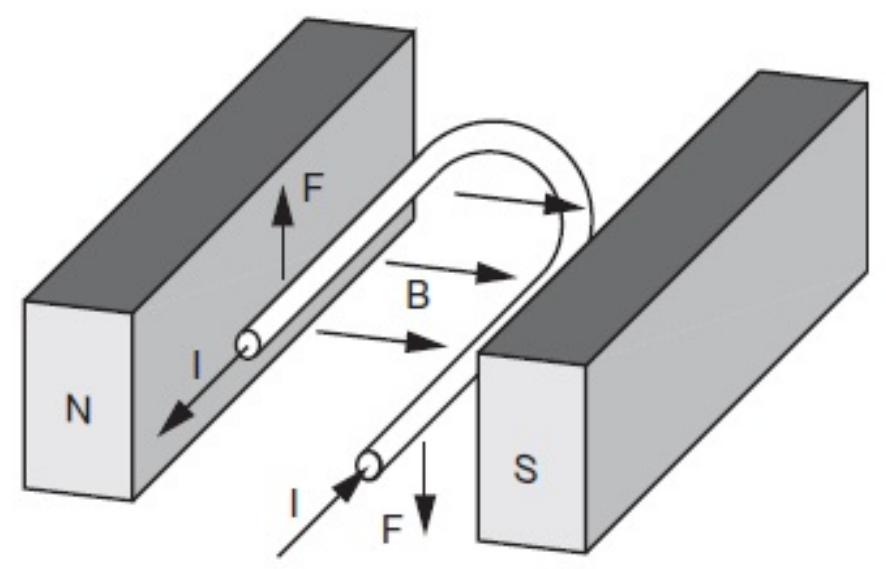

Figure 2.7: A current-carrying loop of wire in a magnetic field, Source: [75] 
$\otimes$ Current into page

$\odot$ Current out of page
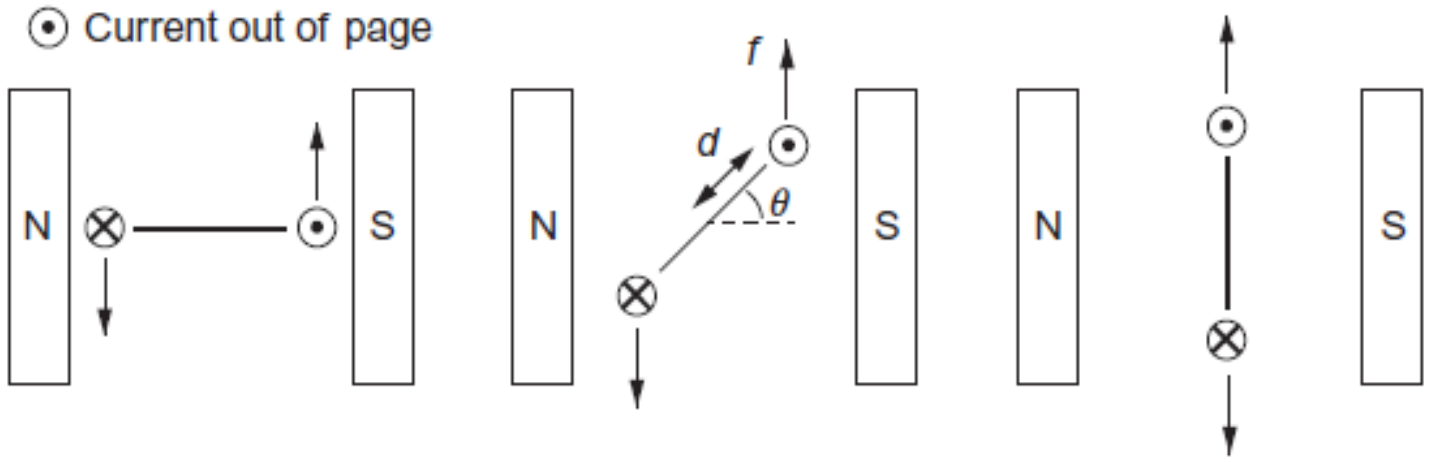

Figure 2.8: Current flows create forces of opposite directions on the loop, Source: [75]

\subsubsection{Governing Equations}

To derive an equation for modelling the motor's performance, we disregarded the commutation details and focused on mechanical and electrical power. The electrical power into the motor is given by:

$$
P_{\text {in }}=I V
$$

Where:

$I=$ the current through the motor.

$V=$ the voltage across the motor.

The motor converts some of the input power to mechanical power. The mechanical power is given by:

$$
P_{\text {mech. }}=\tau \omega
$$

Where:

$\tau=$ the torque of the output shaft.

$\omega=$ the angular velocity of the output shaft. 
Electrically, the motor is defined by the resistance between the two terminals $R$ and inductance due to the coils $L$. The resistance of the motor coils dissipates power $I^{2} R$ as heat. The motor also stores energy $\frac{1}{2} L I^{2}$ in the inductor's magnetic field. The time rate of change of this is $L I\left(\frac{d I}{d t}\right)$, the power into or out of the inductor. Lastly, power is dissipated as sound, heat due to friction at the brush-commutator interface and the bearings. All these factors provide the electrical power put into the motor:

$$
\left.I V=\tau \omega+I^{2} R+L I\left(\frac{d I}{d t}\right)+\text { Power dissipated (friction, sound }, \text { etc }\right)
$$

By disregarding the last term (power dissipated), we have our simple motor model, written in terms of power:

$$
I V=\tau \omega+I^{2} R+L I\left(\frac{d I}{d t}\right)
$$

Dividing both sides of the form (25.2) by I yields will give the following:

$$
V=\frac{\tau}{I} \omega+I R+L\left(\frac{d T}{d t}\right)
$$

Where the relating current to torque (the ratio $\frac{\tau}{I}$ ) is called the torque constant $k_{t}$ and, its given by:

$$
k_{t}=\frac{\tau}{I}
$$

The voltage across a motor's terminals is given by:

$$
V=k_{t} \omega+I R+L\left(\frac{d I}{d t}\right)
$$

The term $k_{t} \omega$ is called the back-emf, where emf is short for electromotive force. Back-emf is the voltage generated by a rotating motor that opposes the input voltage generating the motion [75], [77]. 


\subsubsection{The Speed-Torque Curve}

The Speed-Torque Curve for a motor rotating at constant speed $\omega$ and providing constant torque $\tau$, the current $I$ is constant and, therefore $\frac{d I}{d t}=0$. Under these assumptions, equation 2.9 reduces to

$$
V=k_{t} \omega+I R
$$

Utilizing the definition of the torque constant, we get the equivalent form.

$$
\omega=\frac{1}{k_{t}} V-\frac{R}{k_{t}^{2}} \tau
$$

Equation 2.11 gives $\omega$ as a linear function of $\tau$ for a given constant $V$. This line of slope $-\frac{R}{k_{t^{2}}} \tau$, is called the speed-torque curve for the voltage $V$.

The speed-torque curve plots all the functional constant-current operating conditions with voltage $V$ across the motor.

The maximum speed (at $\tau=0$ ) is called the no-load speed, and the maximum torque (at $\omega=0$ ) is called the stall torque $\tau_{\text {stall }}$ as shown in Figure 2.9. The no-load speed is determined by measuring the unloaded motor speed when powered with the nominal voltage. The nominal is the recommended voltage at which the motor should be operated. It must be assessed to avoid overheating or spinning the motor at speeds beyond the recommended value for the bearings or brushes. Nominal voltage cannot be measured; however, a typical no-load speed for a brushed DC motor is between 3,000 and 10,000 rpm. Therefore, the nominal voltage will regularly give a no-load speed in this range[75], [77], [78].

The speed-torque curve links to constant $V$, but not to constant input power $P_{i n}=I V$.

The current $I$ is linear with $\tau$; thus, the input electrical power increases linearly with $\tau$.

The output mechanical power is given by:

$$
P_{\text {out }}=\tau \omega
$$

The efficiency in converting electrical power to mechanical power is given by:

$$
\eta=\frac{P_{\text {out }}}{P_{\text {in }}}=\frac{\tau \omega}{I V}
$$




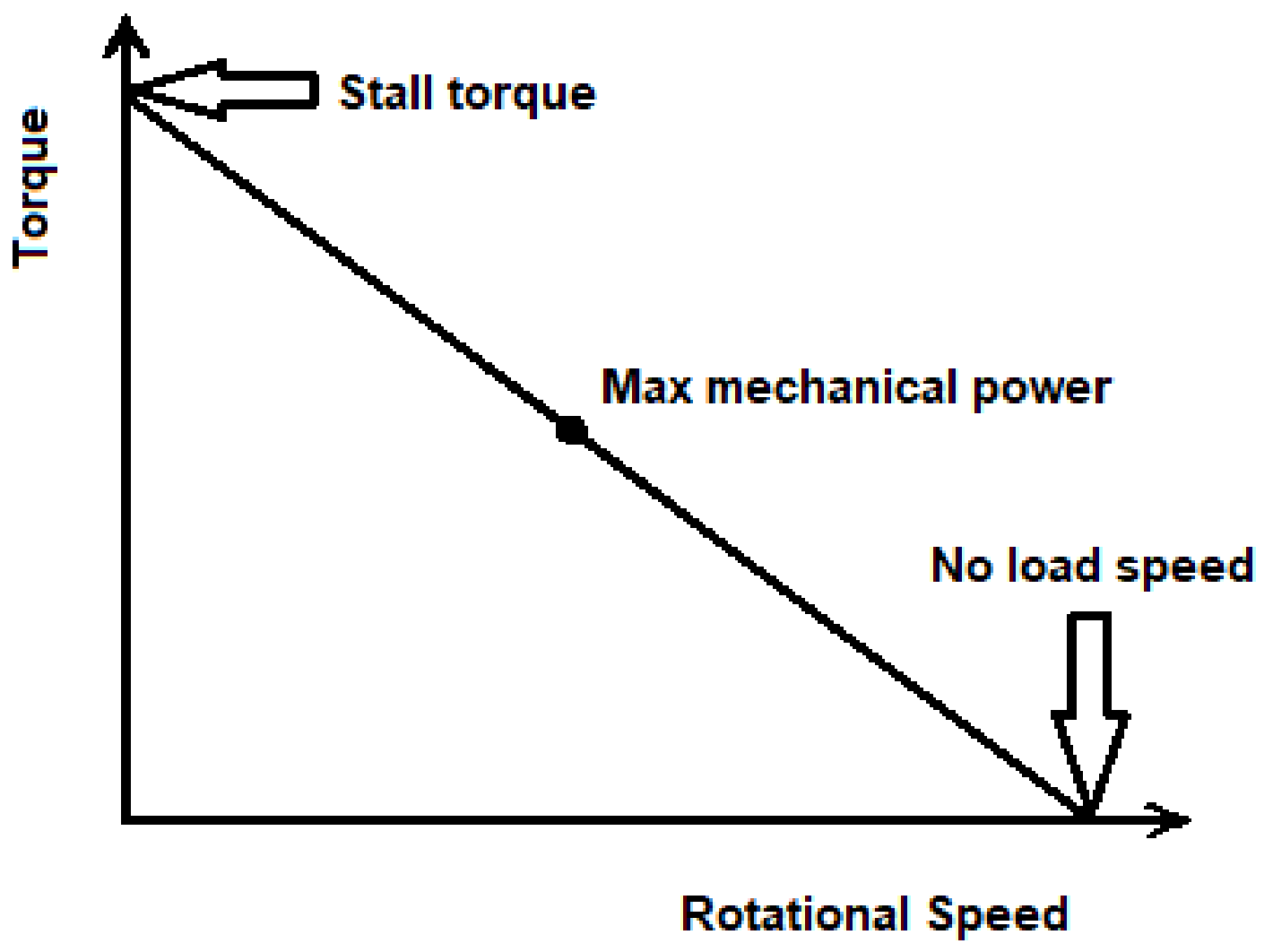

Figure 2.9: DC Motor Speed-Torque Curve, Source: [75] 


\subsubsection{Friction and Motor Efficiency}

Friction and motor efficiency can be determined by assuming that the full torque $\tau=k_{t} I$ generated by the windings is obtainable at the output shaft. In practice, some torque is lost due to friction at the shaft bearings and the brushes [75]. When the motor is spinning, the torque available at the output shaft is given by:

$$
\tau_{\text {out }}=\tau-\tau_{\text {fric }}
$$

The torque delivered to the load is reduced by $\tau_{\text {fric }}$.

By taking friction into account, the motor's efficiency in converting electrical power to mechanical power is given by:

$$
\eta=\tau_{\text {out }} \frac{\omega}{I V}
$$

\subsubsection{Experimentally Characterizing a Brushed DC Motor}

There are many types of instruments and equipment used to experimentally characterize a brushed DC motor. The understanding of the functions and fundamental principles of these instruments and equipment, as well as correctly using them, is essential for the success of motor testing. A dynamometer, sometimes called dyno, is one of the critical devices in electric motor testing; it has been used widely to measure rotating speed, torque, and power output from power sources. Based on the measured operation characteristic data, motor efficiency, and other useful information can be determined. Dynamometers can operate in two basic modes:

1. The absorbing or passive mode in which a dynamometer is driven by the motor under test and implements a specific brake torque load to the testing unit.

2. The driving mode in which a dynamometer drives a machine determines the torque and power required for operating such a driven machine. 
A dynamometer that can both drive or absorb is called a universal dynamometer. Motor testing dynamometers use braking or drag resistance to motor rotation under various operation conditions. Consequently, the absorbing dynamometer is used to absorb the power generated by the motor in motor testing [68].

The brake power $P_{b}$, generated by a dynamometer and applied to the tested motor is calculated using the following equation:

$$
P_{b}=\tau_{\text {dyno }} \omega_{\text {dyno }}
$$

Where:

$\tau_{\text {dyno }}=$ the torque of the dyno

$\omega_{\text {dyno }}=$ the angular velocity of the dyno

In this project, an inertia brake dynamometer (absorption only) was used to test the printed electric motors. All brake types of dynamometers measure power from the torque reaction of the brake at a specific speed. An inertia brake dynamometer uses the test motor to accelerate a flywheel from a standing start-up through the motor speed range. This process involves measuring the change in the flywheel speed as the motor accelerates. The kinetic energy stored in the rotating flywheel is proportional to the square of the rotating speed. Consequently, the flywheel has a significant amount of inertia to resist any change in speed. An inertia dynamometer provides a fixed inertial mass load, calculates the power required to accelerate that fixed and known mass, and uses a computer to record RPM and acceleration rate to calculate torque. The motor is commonly tested from slightly above idle to its maximum RPM, and the output is measured and plotted on a graph [75].

\subsubsection{Permanent Magnets (PMs)}

The presentation of strong rare-earth-based permanent magnets has played an essential role in compacting electric motor design. As of today, PM motors represent the favoured choice when compactness and high torque density are wanted [79]. As shown in the schematics of Figure 2.10[80], a permanent magnet retains its polarisation after the magnetizing field is removed. In order for this to be achieved, a hard magnet should have a high retentivity and a strong coercivity. These properties are accomplished by a perfect crystallographic texture, a high magneto-crystalline anisotropy, and a high saturation polarisation of the alloy. The main classes of hard magnets are Aluminum, Nickel and Cobalt (Alnico), Ceramic Ferrite Magnet, and rare-earth-based magnets (Nd-Fe-B), which are the solution characterized by the most significant energy and high resistance to demagnetization. The 
rare-earth permanent magnets represent the preferred solution [81]. Conventional manufacturing methods for permanent magnets are sintering, pressure Bonding, and injection Molding. Bonded magnets are created of granular magnet material and non-magnetic polymeric material. Hence they are less appropriate for applications where a high energy product is required.

For this reason, the permanent magnets for use in electric motors are created by sintering the granular magnet material. This technique, in turn, raises significant challenges due to the highly abrasive nature of the permanent magnet materials. The strong crystallographic texture is obtained by anisotropic precipitation after severe cold forming, or by anisotropic grain growth during hot deformation or through many other metallurgical processes.[81]. 


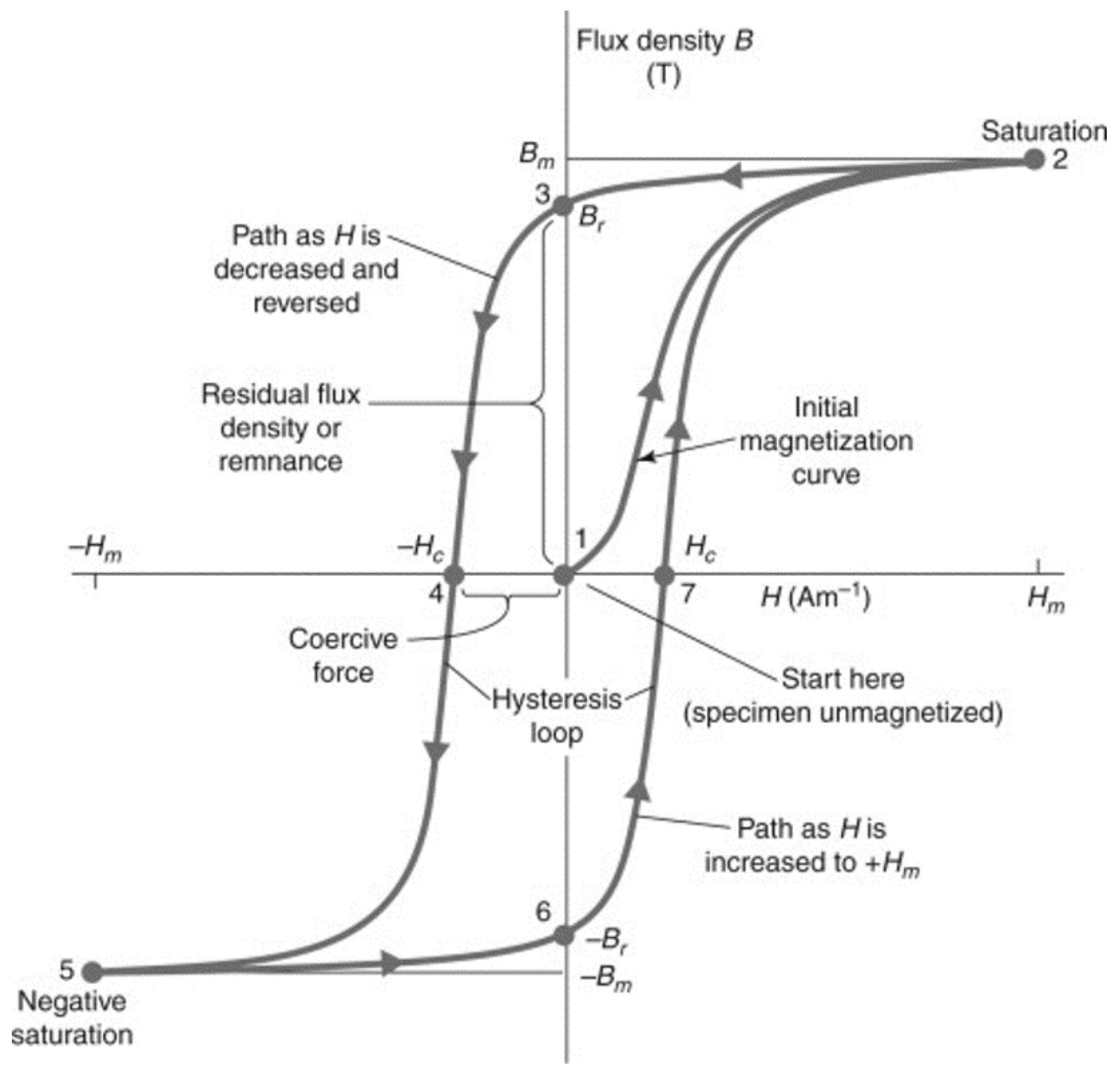

Figure 2.10: Magnetic hysteresis curves for typical ferromagnetic materials, Source: [82]

These various materials properties can accommodate a wide variety of application requirements. A practical overview of factors that must be considered in selecting the proper material of a magnet for a specific application is presented in Table 2.2. The table shows the critical characteristics of selected grades of various materials for comparison[83], [84]. 
Table 2.2: Magnet Material Comparisons, Source: [84]

\begin{tabular}{|c|c|c|c|c|}
\hline Material & Grade & $B r$ & $H c$ & $B H_{\text {Max }}$ \\
\hline \hline NdFeB & $39 \mathrm{H}$ & 12,800 & 12,300 & 40 \\
\hline SmCo & 26 & 10,500 & 9,200 & 26 \\
\hline NdFeB & B10N & 6,800 & 5,780 & 10 \\
\hline Alnico & 5 & 12,500 & 640 & 5.5 \\
\hline Ferrite & 8 & 3,900 & 3,200 & 3.5 \\
\hline
\end{tabular}

These three essential characteristics of the B-H curve are the points at which it intersects at the residual induction $(\mathrm{Br})$, the coercive force $(\mathrm{Hc})$, and the maximum energy product (BHmax) as illustrated in Figure 2.10. $\mathrm{Br}$ represents the maximum flux the magnet can produce under closed circuit conditions. Hc describes the point at which the magnet begins to be demagnetized under an externally applied magnetic field. BHmax represents the point at which the magnetic field's energy density into the air gap surrounding the magnet is at a maximum [83], [84].

\subsubsection{Magnetization Process}

Permanent magnet materials are composed of small domains, each of which exhibits a net magnetic moment. An unmagnetized magnet will possess domains that are randomly oriented to each other, giving a net magnetic moment of zero. Magnetizing fields serve to align randomly oriented domains to give a net, externally observable field [84].

Magnetization process aims to magnetize a magnet to saturation, even if it will later be slightly demagnetized for stabilization purposes. Saturating the magnet and then demagnetizing it in a controlled way ensures that the domains with the least commitment to orientation will be the first to lose their orientation, thereby leading to a more stable magnet. On the other hand, not achieving saturation leads to the orientation of only the most weakly committed domains, consequently leading to a less stable magnet [84].

The magnetization process is accomplished by exposing the magnet to an externally applied magnetic field. This magnetic field is created by currently flowing in coils. The two most common types of magnetizing equipment are DC and capacitor discharge magnetizers. DC magnetizers as illustrated in Figure 2.11, use large coils through which a current is applied for a short duration by closing a switch. The current flowing across the coil produces a magnetic field, usually directed by the use of iron cores and pole pieces, and magnets are situated in the gap between the pole pieces. DC magnetizers are only practical for magnetizing Alnico materials, which have a low magnetizing force requirement, or small sections of Ferrite materials [84], [85]. 


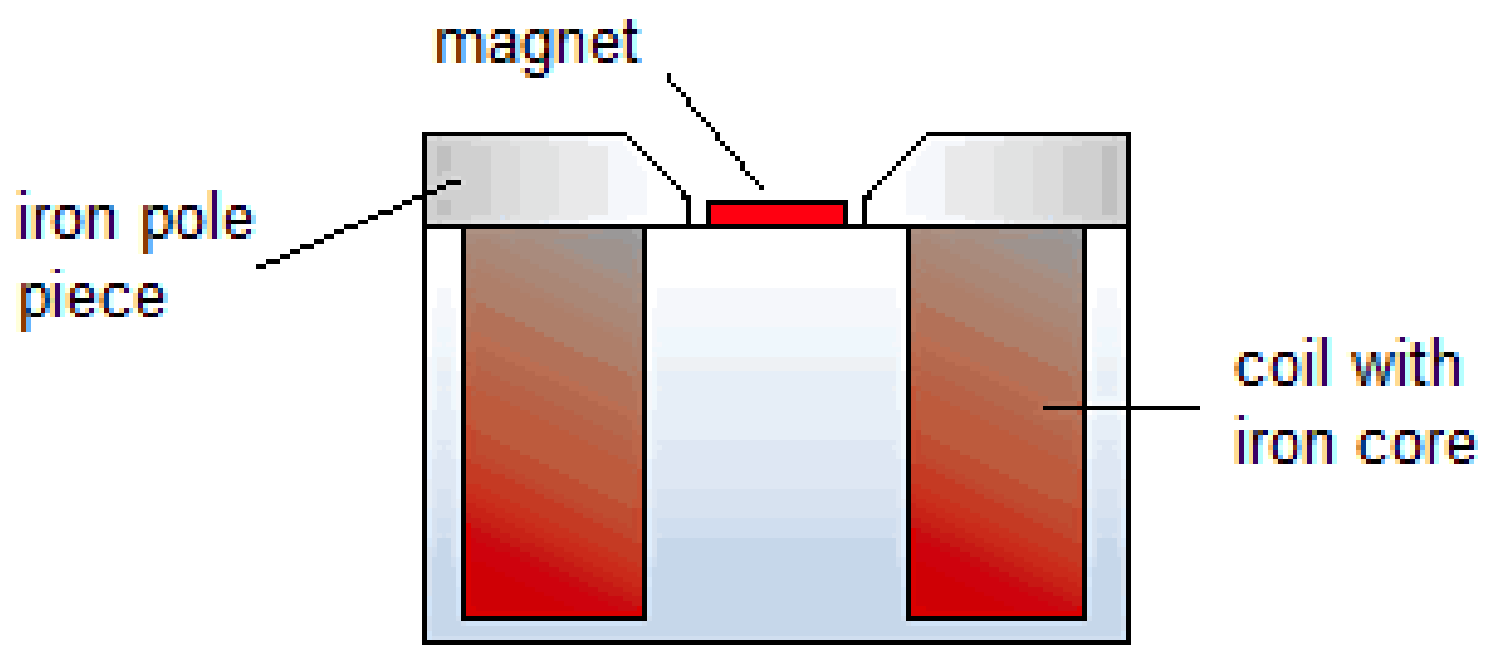

Figure 2.11: DC Magnetizer, Source: [84].

In the case of capacitor discharge magnetizers as illustrated in Figure 2.12, The magnet is exposed to a strong external magnetic field. This method is the most common method of magnetizing, where a very short current pulse goes through a conductor or a coil. The short pulse is generated by capacitor banks that are charged and then discharged through a coil. A high resistivity material can be magnetized with a pulse of a few microseconds, whereas a more conductive material may need a few hundreds of a second longer pulse. Enormously high magnetizing fields in the range of 7959 KiloAmps per meter $(\mathrm{KA} / \mathrm{m})$ can be reached using specific coils and power supplies [86], [87], [84].

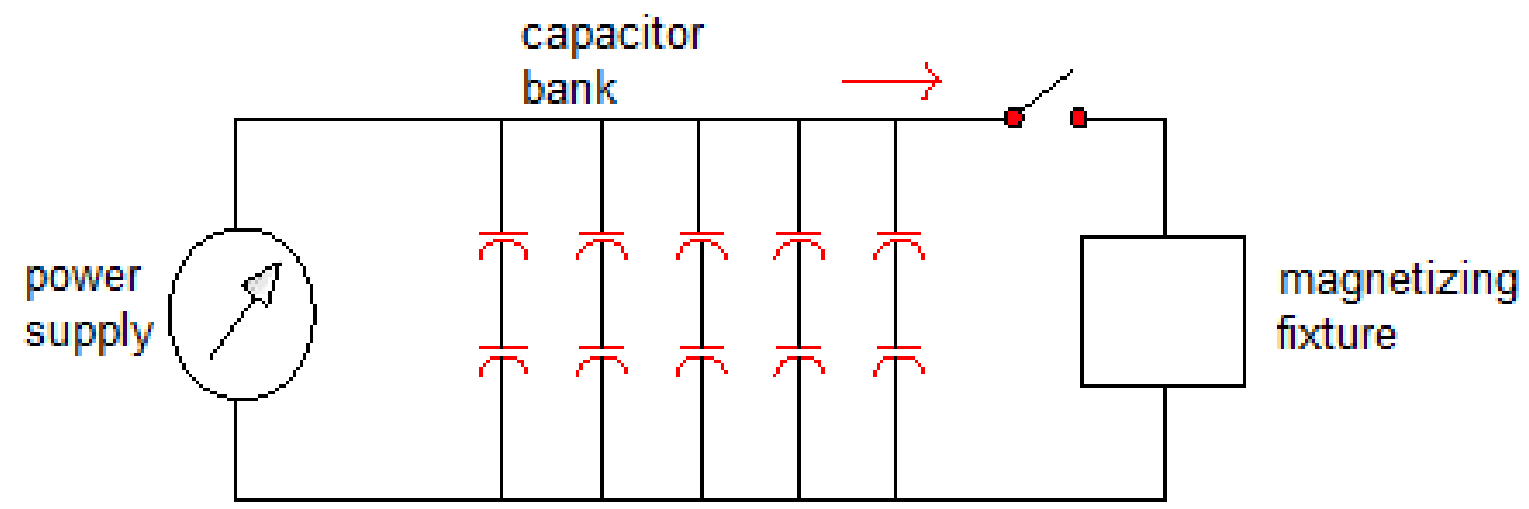

Figure 2.12: Capacitor Discharge Magnetizers, Source: [84] 
Materials that are not attracted to a magnet, such as plastic, brass, or wood, have a permeability of mostly 1. There is no magnetism induced in them by an external magnetic field, and therefore, they are not attracted by a magnet. On the other hand, materials such as Nickel, Cobalt, and Ferrous alloys have high permeability $(\mu)$. Thus, magnetic fields can be induced when subjected to an external magnetic field [88], [89].

$$
B=\mu H
$$

Where:

$B=$ Induced Magnetism in an Alloy

$\mu=$ Alloy's Permeability

$H=$ External Applied Drive Field

There are limits to how much-induced magnetism is workable in different materials and similar workpieces of varying sizes, shapes, and configurations. The concept of magnetic saturation observes that there is a point of diminishing returns at which trying more externally applied magnetic field $(\mathrm{H})$ will result in no additional magnetic induction (B), as shown in Figure 2.13. Increasing the thickness of a permanent magnet is generally helpful. However, these changes will likely influence mass, cost, and possibly easiness of manufacturing. Therefore, it is advantageous to understand and control the magnetic saturation of materials during the design stage. Magnetic permeability is a material's capacity to support an internal magnetic field induced by an external magnetic field [84], [88]. 


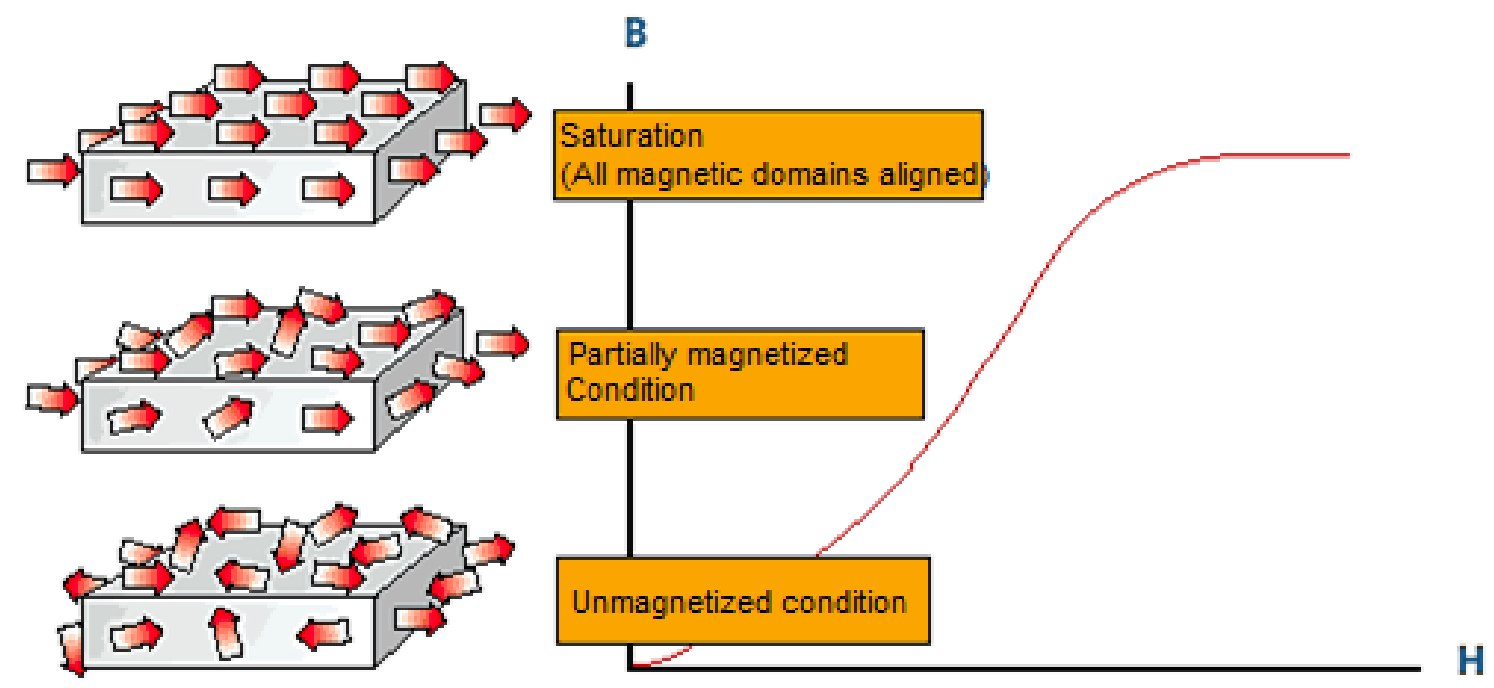

\section{$\mathrm{B}=$ Induced Magnetism \\ $\mathrm{H}=$ External Applied Drive Field}

Figure 2.13: Magnetize a Magnet to Saturation, Source: [84]

Some Rare-Earth magnets need very high magnetizing fields between 1500 to $3900 \mathrm{KA} / \mathrm{m}$ range. These fields are challenging to generate, requiring large power supplies in combination with carefully constructed magnetizing fixtures. Isotropic bonded Neodymium materials need fields in the high $4700 \mathrm{KA} / \mathrm{m}$ range to be completely saturated. Though, fields in the $2300 \mathrm{KA} / \mathrm{m}$ range could achieve $98 \%$ of saturation. Ceramics require fields in the order of $790 \mathrm{KA} / \mathrm{m}$. In comparison, Alnicos require fields in the range of $235 \mathrm{KA} / \mathrm{m}$ for saturation[84], [88].

\subsubsection{Potential for Permanent Magnet Production Using 3D printing Technologies}

The power of AM technology in manufacturing permanent magnets is attracting much interest. Permanent magnets are made of critical and expensive rare earth metals. Recently, powerful magnets are in high demand for electric cars and alternative energy. 3D printing can help reduce costs on materials as it only uses the amount of material required, with little or no wastage and without the time or expense of tooling. These advantages also allow for bringing new designs to the market rapidly. The most powerful permanent magnets should have an organized granular structure that is a challenge to rebuild with a 3D printing machine. All materials have electrons, and electrons have a spin that creates a small magnetic field; thus, each electron is like a tiny magnet. In 
ferromagnetic materials such as iron, unpaired electron spins favour being aligned; thus, their magnetic fields add together instead of cancel out.

Interestingly, in unmagnetized iron, this alignment of spins and their microscopic magnetic fields spread through the entire piece of material. The result is that the material is separated into sub-millimetre magnetized regions, known as magnetic domains. Inside each magnetic domain, the whole unpaired spins are aligned. When a piece of iron is unmagnetized, these domains exist but are randomly oriented and create a net-zero magnetic field. If subjected to a strong magnetic field, the domains will align, and the piece is magnetized with the south and north pole. The elements in the periodic table that are ferromagnetic at room temperature are cobalt, iron, and nickel. Permanent magnets are based on these three elements combined with other elements, such as samarium and neodymium, to change their properties. PMs, keep their magnetized state, attracting materials like iron and steel regardless of electrical current and surroundings. For materials magnetization, a strong field and energy are required to adjust their magnetic domains' direction in order to keep their magnetization permanently. This alignment can be achieved using a strong magnetic field or when the powder is exposed to high pressure. After the grains are aligned, they are locked in place before the magnetization step. This pre-alignment process results in a stronger magnet since all of the crystal's points in the favoured direction contribute to the resulting magnetic field. While, the manufacturing process of isotropic magnets, creates unmagnetized bodies with grains pointed in every direction randomly. In this situation, only a fraction of the grains can contribute, and the resulting magnet is weaker than anisotropic magnets. However, it is a short and straightforward manufacturing process, which also means that any direction of magnetization is possible. Sintered magnets are formed using solid magnetic material with pressure and temperature with no filler particles or bonding agents. Neodymium magnets are the most powerful sintered anisotropic magnets. Bonded magnets are formed by using epoxy or nylon to stick to the magnetic grains. The bonding process allows for more complicated designs and mass production techniques similar to injection moulding; However, the bonding substance reduces the strength and the density of the magnet. Although bonding technology does not establish the strongest magnets, the basis for most current research in 3D printing magnets depends on this type of manufacturing process due to the similarities between both approaches. The first publications detailing 3D printed magnets originates from the Vienna University of Technology. They demonstrated the theory that 3D printed magnets are possible, utilizing custom magnetic filament and a basic printer [90].

Researchers from MIT have combined a magnetic 3D printing ink and magnetic field employed during the extrusion process to manage the magnetic directions of 3D printed regions of soft magnetic materials. The process does not produce a magnet, but their innovation may point toward future developments in 3D printed magnets [91]. 
Oak Ridge National Labs (ORNL) is utilizing additive manufacturing to manufacture 3D printed NdFeB permanent magnets. Instead of using filament melting, which is too slow to keep up with the deposition rates needed to create large magnets. ORNL directly extrudes pellets to form magnets over 5 inches in diameter at high speed [92].

This process is limited to isotropic magnets. However, researchers at ORNL have expanded the work to involve a post-printing alignment step for anisotropic powders. This method makes them closer to competing with existing injection-moulded magnets while offering a large scale and tool-less advantages of additive manufacturing[93].

The magnetic strength is strongly affected by the higher concentrations of magnetic material and the plastic binders' lower concentrations. This, along with the shift to anisotropic materials, are under development at ORNL. Therefore, it seems that, despite the potential interest of hard magnet processing using 3D printing technology, the technical challenges lie in matching the performance of the most advanced magnets produced by traditional methods. In this project, due to the lack of access to sintering and magnetization devices, we decided to use a third party to provide us with the 3D printed permanent magnets. Consequently, four 3D-printed PMs were built from $\mathrm{NdFeB}$ by the Oak Ridge National Laboratory were used to construct the fully 3D printed motor. [94] 
Table 2.3: Differences between Hard and Soft Magnetic Materials, Source: [95]

\begin{tabular}{|c|c|}
\hline Hard Magnets & Soft Magnets \\
\hline $\begin{array}{l}\text { Materials which retain their magnetism and are difficult } \\
\text { to demagnetize are called hard magnetic materials. }\end{array}$ & $\begin{array}{l}\text { Soft magnetic materials are easy to magnetize and } \\
\text { demagnetize. }\end{array}$ \\
\hline $\begin{array}{l}\text { These materials retain their magnetism even after the } \\
\text { removal of the applied magnetic field. Hence these } \\
\text { materials are used for making permanent magnets. }\end{array}$ & These materials are used for making temporary magnets. \\
\hline $\begin{array}{l}\text { They have large hysteresis loss due to the large } \\
\text { hysteresis loop area. }\end{array}$ & $\begin{array}{l}\text { They have low hysteresis loss due to small hysteresis } \\
\text { area. }\end{array}$ \\
\hline Susceptibility and permeability are low. & Susceptibility and permeability are high. \\
\hline Coercivity and retentivity value are large. & Coercivity and retentivity value are less. \\
\hline Magnetic energy stored is high. & $\begin{array}{l}\text { Since they have low retentivity and coercivity, they are } \\
\text { not used for making permanent magnets. }\end{array}$ \\
\hline They possess a high value of $\mathrm{BH}$ products. & Magnetic energy stored is less. \\
\hline The eddy current loss is high. & The eddy current loss is less because of high resistivity. \\
\hline $\begin{array}{l}\text { Example } \\
\text { Carbon Steel } \\
\text { Tungsten-Steel } \\
\text { Cobalt-steel } \\
\text { Copper nickel-iron alloy } \\
\text { Etc }\end{array}$ & $\begin{array}{l}\text { Example } \\
\text { Iron } \\
\text { Si-Steel } \\
\text { Alloy steel } \\
\text { Soft ferrites } \\
\text { Etc }\end{array}$ \\
\hline 1. Used in measuring instruments & 1. Construction of transformer core \\
\hline 2. Magnetic detectors & 2. The core of electrical machines \\
\hline 3. Permanent Magnet & 3. Core of reactors \\
\hline \multirow[t]{2}{*}{ 4. Etc } & 4. Making electromagnets \\
\hline & 5. Etc \\
\hline
\end{tabular}

\subsubsection{Soft Magnetic Cores}

Soft magnets are made of ferromagnetic materials that almost totally lose their magnetization when the applied magnetic field is cut down to zero. In other words, they are characterized by a thin induction-applied field loop (B-H loop). A soft magnetic material magnetization curve is illustrated in Figure 2.14, where the main properties are also shown. The differences between hard and soft Magnetic Materials are presented in Table 2.3, which reports the fundamental electromagnetic and mechanical properties [96]. A good core soft magnets properties can be described as following:

Permeability $\mu$ : High magnetic permeability allows the core to reach high inductions (B) at low applied fields $(\mathrm{H})$, increasing the motor's efficiency.

Saturation: High saturation magnetization allows the material to achieve high B. Consequently, a higher B 
produces higher torque, given the same machine design and dimensions.

Coercivity: Low magnetic coercivity is required to reduce the hysteresis losses (hysteresis losses are equal to the area contoured by the B-H loop).

Resistivity: High electrical resistivity is needed to limit the propagation of the eddy currents induced by a time-varying B inside a core.

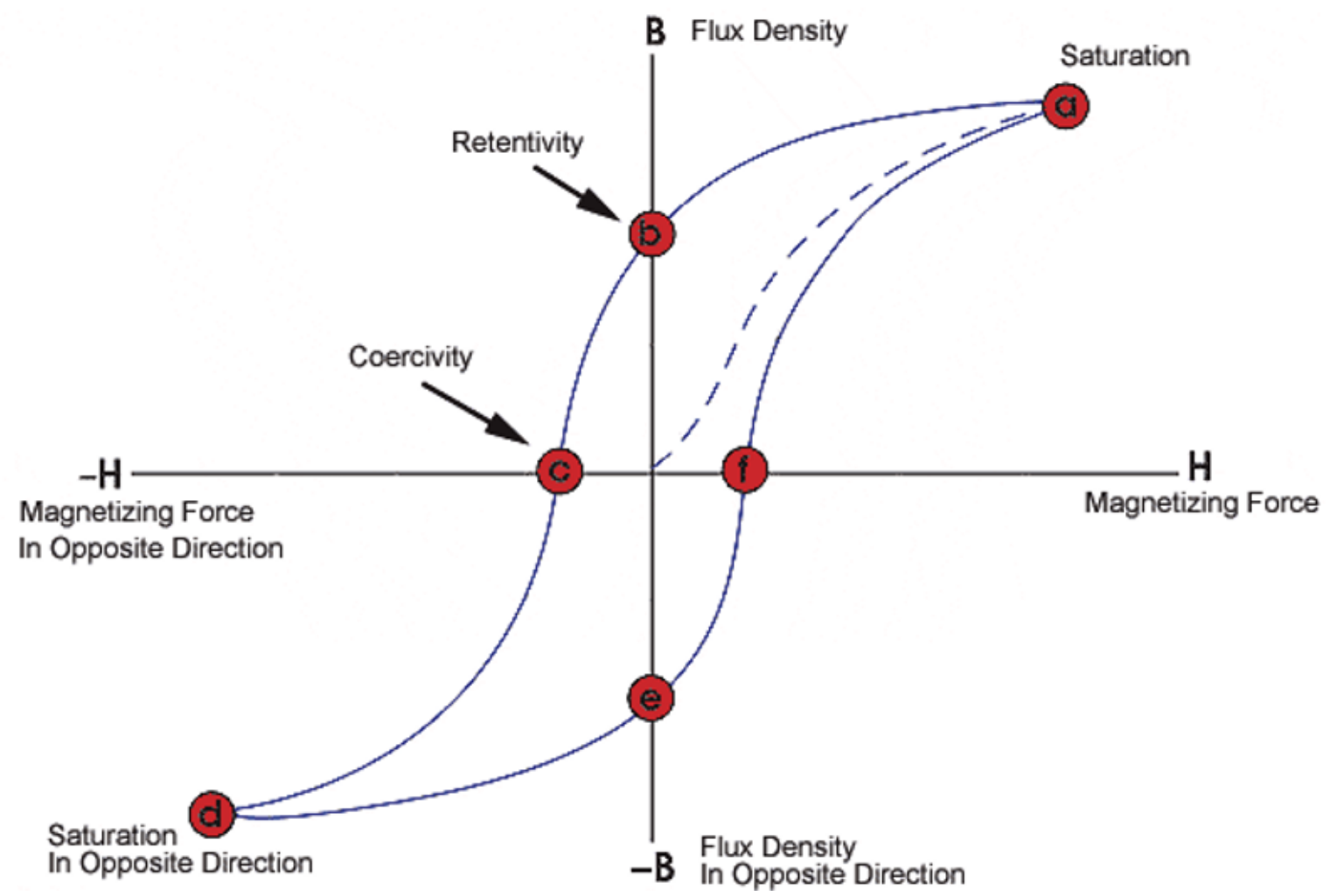

Figure 2.14: Soft Magnetic Material Magnetization Curve, Source: [19]

As an alternative to the use of high-resistivity materials, which often lack excellent ferromagnetic and mechanical properties, the propagation of eddy currents is usually limited by constructing the cores of the number of stacks varnish-insulated laminations, typically 0.1 to $0.5 \mathrm{~mm}$ thick, as illustrated in Figure 2.15. The translational symmetry that lamination imposes on designs can also be avoided using soft magnetic composites (ferromagnetic powder particles surrounded by an electrical insulation film) to reduce eddy-current propagation [97]. 


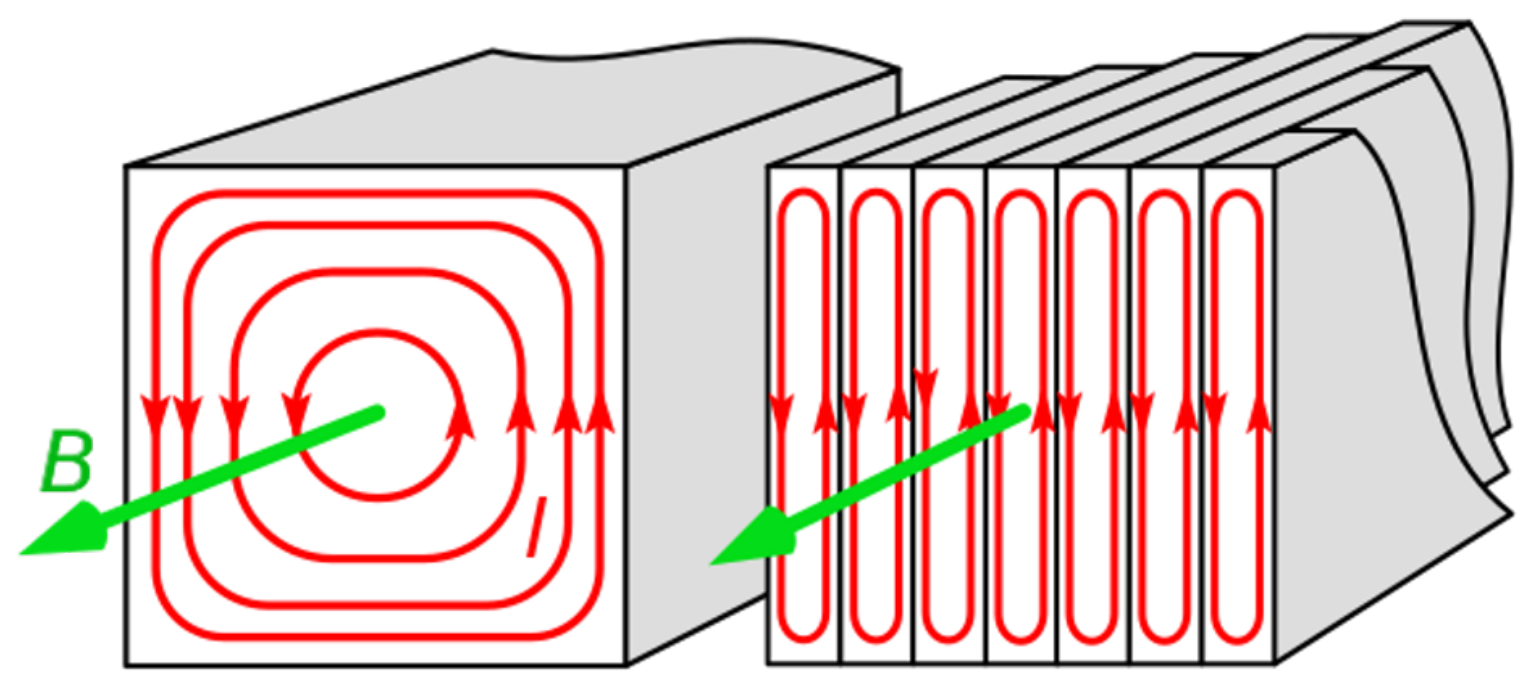

Figure 2.15: Eddy currents in laminated cores (right) are smaller than those in solid cores (left), Source: [19].

\subsubsection{Silicon steel (Fe-Si)}

Silicon steel (concentration range 2-6.5 wt \%) is the best available solution for core creation, due to the attractive electromagnetic properties of high resistivity, high permeability, low hysteresis losses, and thermal stability. Laminated Fe-Si alloys are often described as electrical steels [98]. Importantly, within the family of silicon steels, $\mathrm{Fe}-6.5 \mathrm{wt} \% \mathrm{Si}$ has a high potential in magnetic device applications due to its very low eddy-current and hysteresis losses [99]. However, the high silicon content resulting in severe brittleness that forbids mass production of high silicon steel using the economical conventional processing methods, which leads to high processing costs and limitations of size and shape [100].

\subsubsection{Iron-Cobalt alloys ( $\mathrm{Fe}-\mathrm{Co})$}

Mainly, in the equiatomic composition form (i.e. Fe-50wt\%Co alloy), these alloys are characterized by the highest saturation magnetization and temperature of transition from ferromagnetic to paramagnetic, therefore making them mainly valuable in applications where compactness and thermal stability are required [81]. 


\subsubsection{Nickel-Iron alloys ( $\mathrm{Fe}-\mathrm{Ni})$}

Fe-Ni alloys are precious in their face-centred cubic state for a reason of their very high permeability. While their employment is mostly limited to a few regions of Ni content (particularly $80 \mathrm{wt} \%$ and $50 \mathrm{wt} \%$ ), Fe-80wt $\% \mathrm{Ni}$ offers an extremely high permeability; therefore, it is commonly known as permalloy. Low electrical resistivities and saturation magnetization are the main disadvantages of this kind of alloy [81].

\subsubsection{Soft ferrites}

Iron oxide $\mathrm{Fe} 3 \mathrm{O} 4$ is a ferromagnetic ceramic and presents an electrical resistivity that is several orders of magnitude higher compared to metallic alloys. Therefore, soft ferrites are particularly suitable for high-frequency applications (up to $100 \mathrm{MHz}$ for the NiZn grades). While low saturation magnetizations generally characterize soft ferrites, they can be doped with other oxides, such as $\mathrm{Mno}$ and $\mathrm{ZnO}$, to gain relatively high permeability, comparable with that of Fe-Si [81].

\subsubsection{Soft magnetic composites (SMCs)}

Iron grains can be coated with a polymeric insulator layer to be compacted using powder metallurgy technology. The main advantages of SMCs are the low eddy current losses and the option of extending the design of electric motor core to three-dimensions. A comparison between electric magnetic laminated sheets and soft magnetic composite materials is illustrated in Figure 2.16. However, Soft magnetic composites suffer from low permeability and low saturation magnetization, which limited the use of these new materials to high-frequency applications. 
Electric magnetic laminated sheets

Laminated sheets which are coated by insulating layer

High Joule heat in plane which is perpendicular to the magnetic field

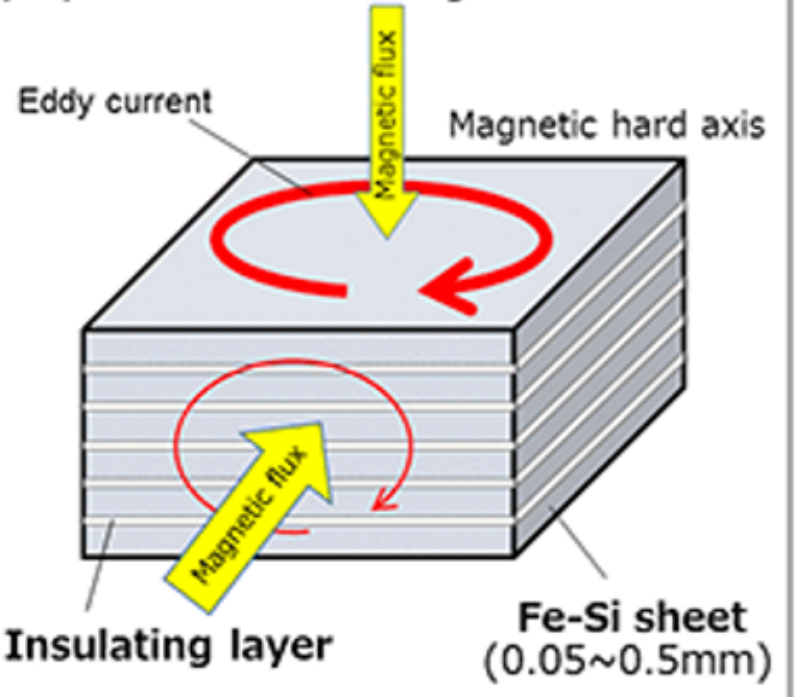

Soft magnetic composite materials

\section{Compacting powders which are covered with insulating film}

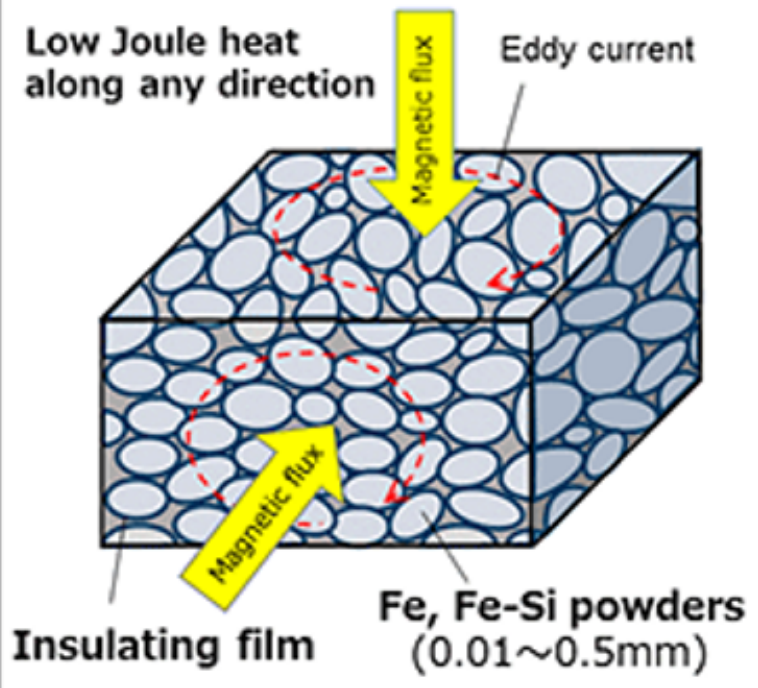

Figure 2.16: Comparison Between Electric Magnetic Laminated Sheets and Soft Magnetic Composite Materials, Source: [101]

\subsubsection{Amorphous Alloys (or Metallic Glasses)}

Amorphous alloys for soft magnetic applications are typically attained using compositions of transition metals (Fe, Co and Ni) and metalloids (usually B and Si). Amorphous alloys provide excellent soft magnetic properties, including high permeability, very low hysteresis losses and higher electrical resistivity, at least three times higher than silicon steel. The saturation magnetization is relatively high for Fe-based amorphous alloys, such as Fe-Si-B [102], [103].

This new class of materials realizes a viable combination of excellent soft magnetic, mechanical and corrosion properties for their unique disordered atomic structure [104]. 


\subsubsection{Electromagnetization Process}

While permanent magnets produce an excellent and sometimes powerful static magnetic field, their strength is yet too weak. We also may need to be able to control the amount of magnetic flux that is present. So to produce a much more reliable and more controllable magnetic field, we need to use electricity. Using a wire wound around a soft magnetic material such as an iron core, we can produce powerful electromagnets for use in many different types of electrical applications. This use of wire creates a relationship between electricity and magnetism that gives us another form of magnetizing called Electromagnetism. Electromagnetism is created when an electrical current flows through a simple conductor such as a length of wire or cable. As current passes along the conductor's, a magnetic field is produced along the entire of the conductor. The magnetic field created around the conductor has a specific direction with both the North and South poles produced being defined by the orientation of the electrical current flowing over the conductor. Thus, it is necessary to establish a relationship between the current flowing over the conductor and the resultant magnetic field produced around it, allowing us to determine the relationship that exists between electricity and magnetism in the shape of Electromagnetism. When an electrical current flows via a conductor, a circular electromagnetic field is generated around it with the magnetic lines of flux forming close loops that do not pass around the entire length of the conductor. The direction of rotation of the magnetic field is ruled by the direction of the current flowing within the conductor, with the corresponding magnetic field created being stronger near to the center of the current-carrying conductor. The method of moving the conductor or changing the magnetic field will induce a voltage and current within the coil as a result; this phenomenon is known as Electromagnetic Induction. It is the fundamental principle of operation of electric motors, generators and transformers. Electromagnetic induction is the creation of an electromotive force (EMF) being made as a consequence of relative motion between a conductor and a magnetic field. It was brought to light in 1831 by Michael Faraday and sets the foundation for electrical generation in power plants, electric motors, transformers, and many more aspects. The equation that mathematically represents "electromagnetic induction is Faraday's Law, which states that any change in the magnetic environment of a coiled wire will cause a voltage (EMF) to be induced"[105]. Faraday discovered many ways to happen, such as moving a magnet through a coil of wire, changing the magnetic field strength, and moving the coil through a magnetic field. The following equation can describe the voltage (EMF) generated in a coil of wire [106], [107], [105], [108]:

$$
E M F=\frac{-d(N \phi)}{d t}=-N \frac{d \phi}{d t}
$$

Where: 
$N=$ Number of turns in the coil

$\phi=$ The magnetic flux through one coil

$\frac{d(N \phi)}{d t}=$ The rate of change of flux linkage in webers per second

This law is known as Faraday's Law and in words it states that the induced emf is equal to the negative of the rate of change of the magnetic flux linking the circuit.

\subsubsection{Potential for Motor Core Production Using AM Technologies}

Electric motor softcore production is limited to silicon steel laminations, which are used to decrease eddy current losses. However, Steel laminations are hard to form into complex designs and generate significant waste during processing. On the other hand, AM technologies are not suitable for thin sheet fabrication, due to the well-known thermal gradient-induced warping and high-surface roughness affecting additively manufactured structures. However, processing insulated iron particles (such as SMCs) could be performed using powder bed systems. However, this should be equipped with a low-power heat-source, to produce particle bonding without prompting the evaporation of the coating layer[109], [110].

It should be noted that the flux variation is minimal in the rotor, accordingly building the rotor as a solid structure is possible. Therefore, the rotor core of the PM electric motors appears to be suitable for fabrication using AM technologies [109].

In soft magnetic materials, the change in composition results in systematic changes in functional and mechanical behaviour. Previous studies on soft magnetic materials have concentrated on thin films, limiting their use to thin film-based components. Limited studies used laser additive manufacturing to produce $\mathrm{Ni}$ alloys [111], $\mathrm{Fe}-\mathrm{Si}$ alloys [112], [113] and Ti alloys [114] have been published. The production of soft magnetic cores utilizing AM might have the potential to discover new materials in an accelerated way as a single sample can provide information about the properties of several different compositions making this a useful combinatorial evaluation tool. The operation of electrical motors at higher speeds and smaller sizes could be facilitated by developing magnetically soft magnetic and mechanically strong materials[115]. Previous studies on producing soft magnetic alloys, including Fe-Ni [116], [117], [118], Fe-Ni-V, and Fe-Ni-Mo [119], [120] show that AM is a promising approach to prepare soft magnetic materials.

3D printing of electric motors is still at an undeveloped stage. This work, thus, aims to provide initial proof of concept of a fully 3D printed DC motor, with the major-specific contribution being the 3D printed soft magnets. For this purpose, two soft-magnetic materials of a ferrosilicon alloy with a silicon content of $3 \mathrm{wt} \%$ and a $6.5 \mathrm{wt} \%$ were selected for processing with the CSAM technique. In the electrical industry, the ferrosilicon alloy is widely 
used as sheet material due to its increased specific resistance. The CSAM technique has been chosen due to its suitability for creating composite components. Magnetic PLA from Proto-Pasta was also selected as the core of an electromagnet for processing with the FDM technique. With its properties, the material could be beneficial to the 3d-print rotor and stator cores for electromagnetic coils. The magnetic behaviour of this material shows that it is much less magnetic than things made of steel [121]. At the same time, the positive side of the Magnetic PLA is non-conductive. Therefore, there is no hazard to create short-cuts when winding the copper coils, and there are no eddy current losses.

\subsection{Relevant Previous Attempts to 3D Print Functional Objects}

Fabrication of functional and interactive objects is a newly developing research area. One approach to this work is to embed off-the-shelf sensors inside a printed object. H. Peng et al. [122] developed a custom print head with two-wire feeders that can deliver the wire within five-degrees of freedom 5DOF on an FDM printer. They used this for winding copper wire coil to induce a magnetic field and winding soft iron over a plastic structure to form highly magnetic permeability poles. They demonstrated this by fabricating a 6-pole motor stepper stator (i.e. a reluctance motor rotor). Savage et al. [123] showed how 3D-printing could be used to build functional objects by embedding a machine vision-based system for sensing human input in physical controls, such as joysticks, buttons and sliders. Hook et al. [124] embedded small three-axis wireless accelerometers in the moving parts of a 3D-printed object to tracking movement.

Furthermore, RevoMaker [125]presented the idea of inserting premade circuits inside a folded cuboid, then printing over its facets representing computer muse. Savage et al. [126] also developed placing scanning marks and stickers on a solid model surface composed of sculpting materials, thereby allowing them to be converted into 3D-printed geometry. They then mounted electronics and sensors inside the 3D-printed item.

Other researchers have created functional objects by adding off-the-shelf sensors and actuator parts during the printing process, including 3D-printed light pipes [127], interactive speakers [128], pneumatic device controls [129], hydraulically actuated robotic structures [130] and capacitive touch sensors for interactive objects [131]. Voxel8 [132] developed an FDM printer that can print with both thermoplastic and conductive silver paste. In addition to embedding off-the-shelf electronic components such as motors, this made printing a complete quadcopter possible, including the circuits.

Researchers have recently investigated different techniques to insert conductive wires into 3D-printed geometry, thus making it possible to produce 3D-printed interactive objects. Kim et al. [133] demonstrated early results 
of embedding a copper wire on an airflow duct built by FDM, to demonstrate multi-material 3D-printed electronics. Bayless et al. [134] studied embedding wire by adding a wire-printing tool head to the RepRap 3D-printer to manufacture hybrid wire/plastic parts.

Bas [135] tested printing over a copper wire using a rotary ring to orient the laid wire and extruding filament to cover it, as an example of single-layer wiring prints.

Skylar-Scott et al. [136] introduced a combination of direct ink writing with a focused laser that anneals printed metallic features locally, to produce complex structures such as helical springs at micro-scale. Finally, Christoph Laimer [137] built the 3D-printed rotor and stator of a brushless DC electric motor with an ordinary FDM printer, then embedded off-the-shelf magnets, copper wire, an electronic speed control (ESC) and ball-bearings.

Fabrication of functional objects using AM is a new research area. The fundamental way is to embed offthe-shelf devices on or in a printed object, including conductive wires, into the printed geometry throughout the printing method. In recent researches, conductive inks were such as silver ink also used to form electronic circuits into 3D printed parts. Although the employment of $A M$ technologies for constructing a wide range of dielectric and metallic materials, previous studies about fabricating electromechanical devices are comparatively limited. All efforts to $3 D$-print electric motors only considered the main structure of the rotor and stator. The little experimentation on the capabilities of AM on the development of products with mixed materials and different technologies, such as circuit boards and electric motors, shows a gap in the literature.

\subsection{Research contributions}

This study presents a new approach to creating a fully 3D printed electric motor, including all motor parts such as the rotor core with the field winding, the stator core with stator winding or permanent magnets, insulation and the outer frame. We examine how to develop an electric motor with multi-material and multi-technique to attain a 3D printed motor that can be built without embedding devices. We are also looking at performance testing against benchmark components. 


\section{Chapter 3}

\section{Methodology: Fabricating and Testing of}

\section{Radial-Flux DC Motor}

An electric motor is essentially a rotor and a stator, separated by an air gap. The rotor is the rotating section and carries the winding, while the stator is stationary and holds the permanent magnets (PMs). The rotor is typically made of silicon steel, which is available in thin lamination sheets, and cut by laser to form the desired patterns. The sheets are connected to form the final shape of the rotor cores [68]. In this study, the brushed DC motor (BDCM) was chosen to be studied and 3D-printed because the electric power source in a classical BDC motor is connected to the rotor winding through a commutator and brushes. So there is no need for electronic commutation based on hall position sensors [68].

Different approaches were used in this study to achieve a fully 3D-printed motor. Some of the motor parts such as rotor, stator and commutator were done using a RepRap machine based on FDM. The RepRap consists of a combination of printed mechanical components, stepper motors, extrusion and a hot-end for melting and depositing sequential layers of polymers. An open-source Melzi V2.0 micro-controller controls it. The extruder uses a filament of the material, such as PLA or ABS. The Replicating Rapid Prototyper (RepRap) machine melts the filament and extrudes it through a nozzle to form the desired shape. The printer has a building volume of $210 \mathrm{~mm}(\mathrm{~W}) \times 190 \mathrm{~mm}(\mathrm{D})$ x $140 \mathrm{~mm}(\mathrm{H})$ and a building speed of $1,800 \mathrm{~mm} / \mathrm{min}$, a $0.5 \mathrm{~mm}$ nozzle, positioning accuracy of $0.1 \mathrm{~mm}$ and a resolution of $0.0125 \mathrm{~mm}$. All 3D models were created using PTC Creo CAD software, and the files were then converted to STL format. The communications software Pronterface is used to talk to the RepRap machine, using a G-code file format that the printer understands. Before a model can be processed, a slicing tool is required, and Slic3r software is used. It took the solid model file (STL format) and sliced it into 
layers [138].

Some of the 3D-printed parts in this project were created using Anet A8 machine, a desktop 3D-printer from Shenzhen Anet Technology Co. Ltd. It is based on the Prusa i3 design and is the most widely used FDM 3D-printing technology. This printer has a building volume of $220 \mathrm{~mm}$ (W) x $220 \mathrm{~mm}$ (D) x $240 \mathrm{~mm}(\mathrm{H})$, and a printing speed of $40-120 \mathrm{~mm} / \mathrm{s}$. The Anet A8 has a $0.4 \mathrm{~mm}$ nozzle, positioning accuracy of $0.1 \mathrm{~mm}$ and a resolution of \pm 0.1 to $0.2 \mathrm{~mm}$. Cura software was used as the slicing tool [94], the software to design the prototypes for the Anet A8 FDM printer was PTC Creo, and all filament diameters were $1.75 \mathrm{~mm}$.

\subsection{Materials and Methods Used in the Manufacture of Rotor Core}

The first approach focused on 3D-printing and only tested the rotor of the BDC motor while retaining the conventional stator constructed of two NdFeB PMs. The 3D-printed rotor core (see Figure 3.1a) was 3D-printed by the RepRap using magnetic iron PLA filament (see printing parametrs of magnetic iron PLA filament in appendix B). Eight equally spaced slots were created at the rotor core's outer circumference and used for winding the copper coil. Four poles were wound with copper coil $30 \mathrm{MAG}(0.16 \mathrm{~mm}$ diameter), and the number of turns per pole was 95. The commutator was also 3D-printed using ABS filament on the RepRap machine (see printing parametrs of ABS filament in appendix B), and four sets of contact copper sheets $(0.254 \mathrm{~mm})$ were attached to the commutator. The rotor core and the commutator were attached to a steel axle of $3.175 \mathrm{~mm}$ diameter and $20 \mathrm{~mm}$ long, and the ends of the winding coils were soldere $\mathrm{d}$ to the commutator copper tapes by hand. The electric power source was connected to the rotor winding through the commutator and brushes, with two stationary brushes contacting the copper conductor tapes of the commutator in order to reverse the flow of current in the rotor. Two copper wires of $20 \mathrm{GA}$ were used to form the brushes.

Electric steel is the most widely used soft magnetic material for constructing electric motors due to its high permeability [139]. Therefore, We implemented another procedure to create two other rotors using silicon steel particles (Fe-Si) with two different compositions: Fe-3Si and $\mathrm{Fe}-6.5 \mathrm{Si}$, as shown in Figures $3.1 \mathrm{~b}$ and c, respectively. The two Fe-Si cores were printed using a Cold Spray Additive Manufacturing (CSAM) technique [140]. Both electric-steel 3D-printed cores were provided by the National Research Council of Canada (NRC); the diameter of each core was $40 \mathrm{~mm}$, with a height of $14 \mathrm{~mm}$. Both cores were covered with two 3D-printed thin disks, and PLA filament with a diameter of $1.75 \mathrm{~mm}$ was used to print the disks in order to create the required slots at the outer circumference. Four of the slots were used to wind the copper coil with 95 turns in the first rotor, as shown in Figures 3.1b and c; the same number of turns allows for superior comparisons. 


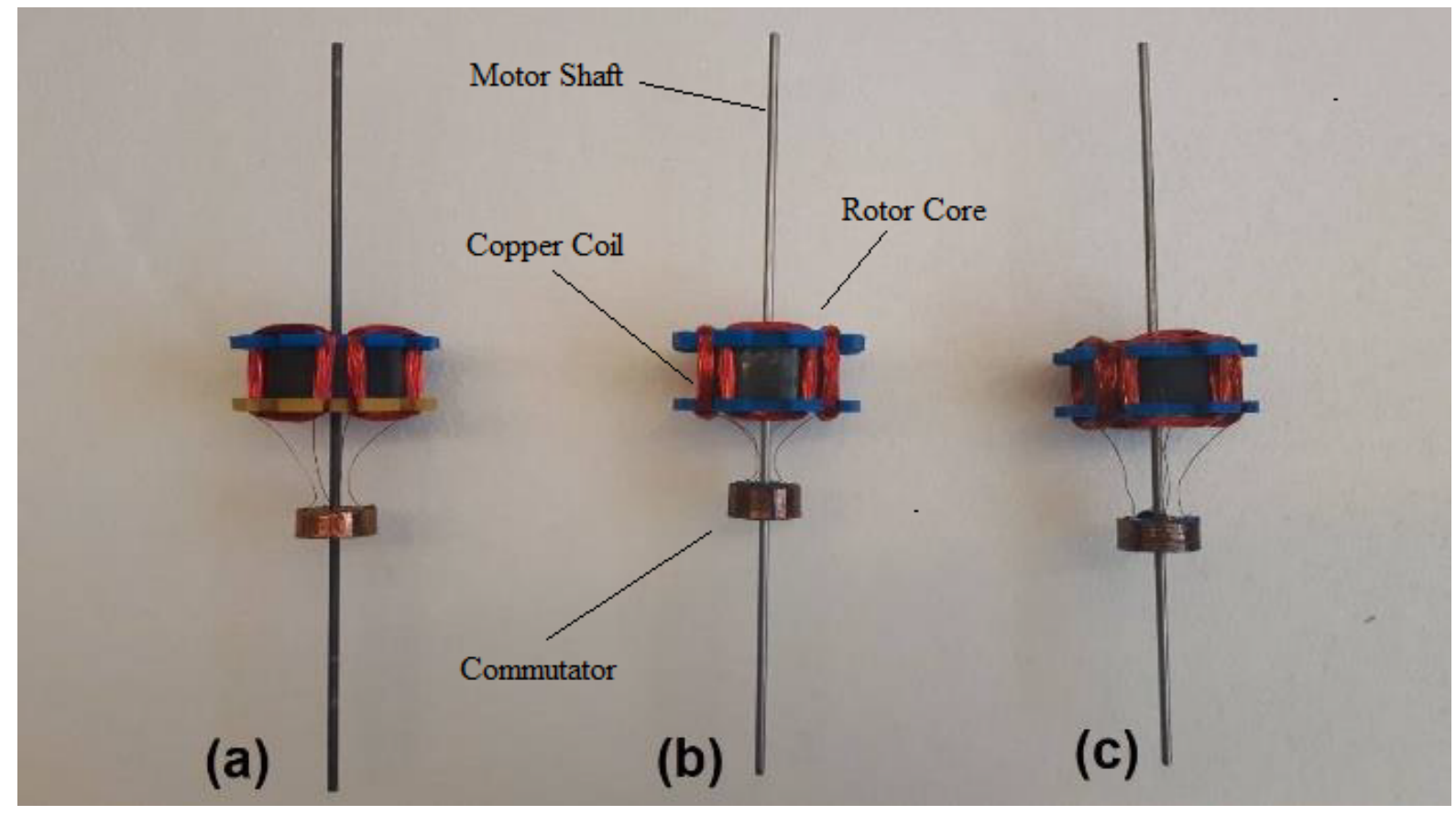

Figure 3.1: DC electric motor rotor (a) Magnetic Iron (b) Fe-3Si (c) Fe-6.5Si

\subsubsection{Results and Discussion}

The three motors were assembled and connected to $15 \mathrm{~V}$ DC power. The stator winding was consecutively energized. A rotating magnetic field was generated, and the rotor aligned itself with the magnetic field of the two different poles of the PM, all of which led to the successful rotation of the three rotors. The evaluation consisted of comparing RPM vs. time and torque vs. RPM. Testing was done by the electric motor dynamometer (dyno) [141], which measures the rpm, voltage, current draw, power and torque output of electric motors. The setup of the motors was modified to be compatible with the dyno test base and was done by 3D-printing two motor holders, as shown in Figure 3.2. 


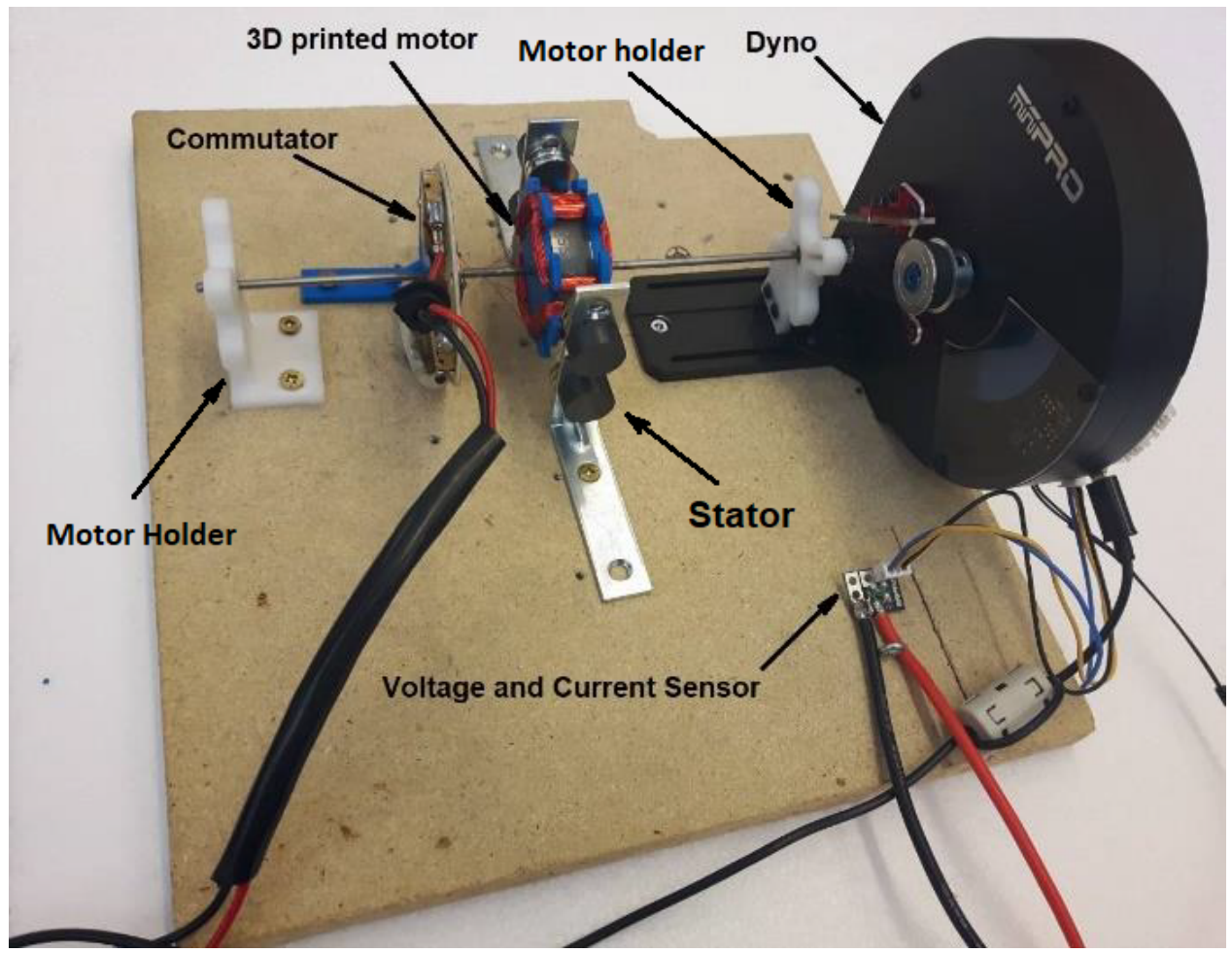

Figure 3.2: 3D printed DC motor connected to electric motor dyno

The graph in Figure 3.3 shows the RPM versus the time of the three tests. The magnatic iron PLA motor accelerates from 0 to 2089 RPM in about 8.1 seconds; this point represents the maximum RPM as soon torque and power reach zero. In comparison, the Fe-6.5Si motor reaches its maximum speed $1632 \mathrm{RPM}$ in about 10.4 seconds. While the Fe-3Si motor performance was less than the two prvious mentioned motors in terms of its maximum speed; it reaches its maximum speed 738 RPM in about 6.1 seconds. The speed-torque curve illustrated in Figure ?? is considered an essential curve. It demonstrates the relationship between speed and torque. The speed achieves a maximum when the torque produced by the motor is zero. The data in this graph was plotted using spline interpolation method. 


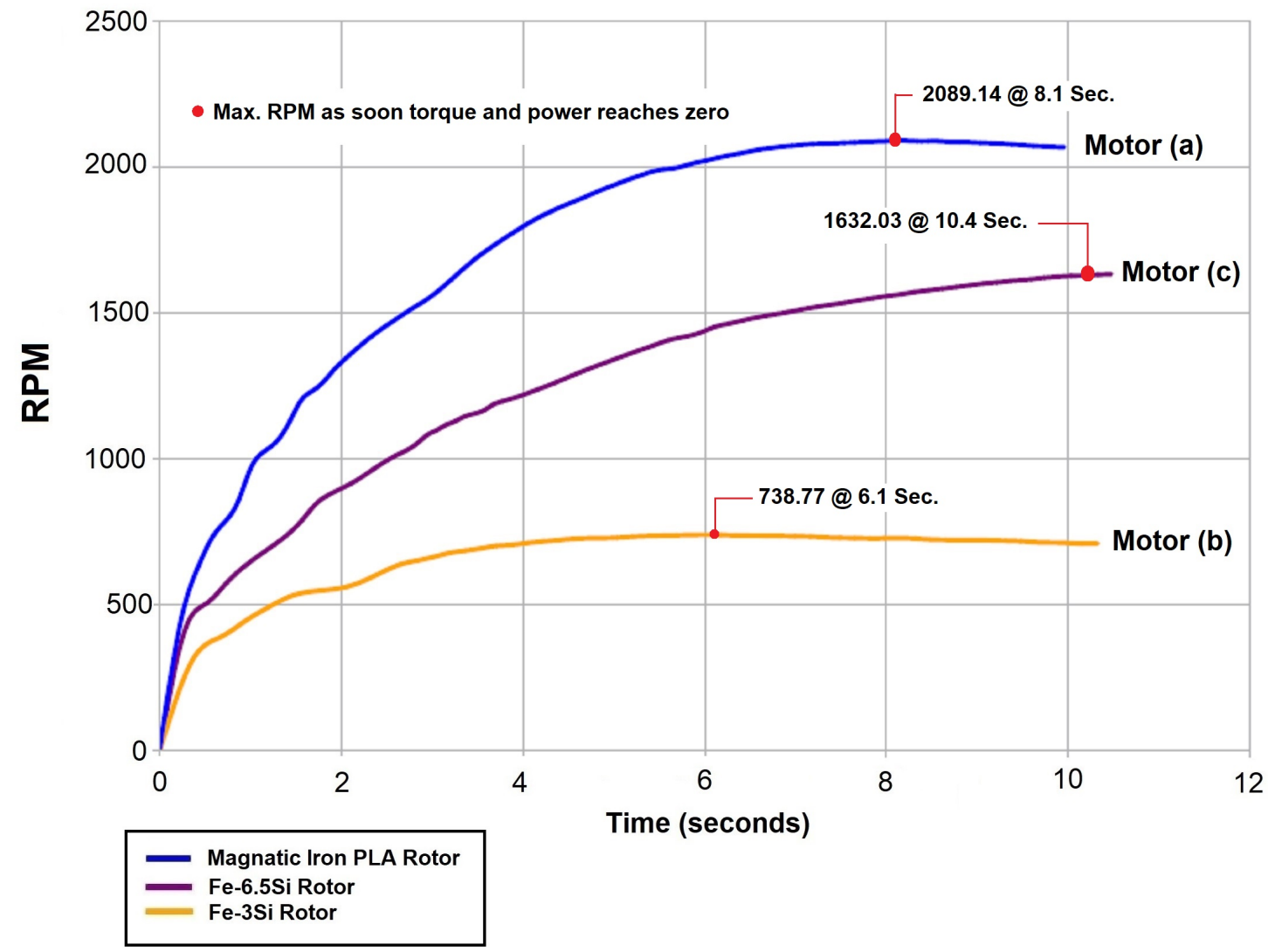

Figure 3.3: RPM Comparison 


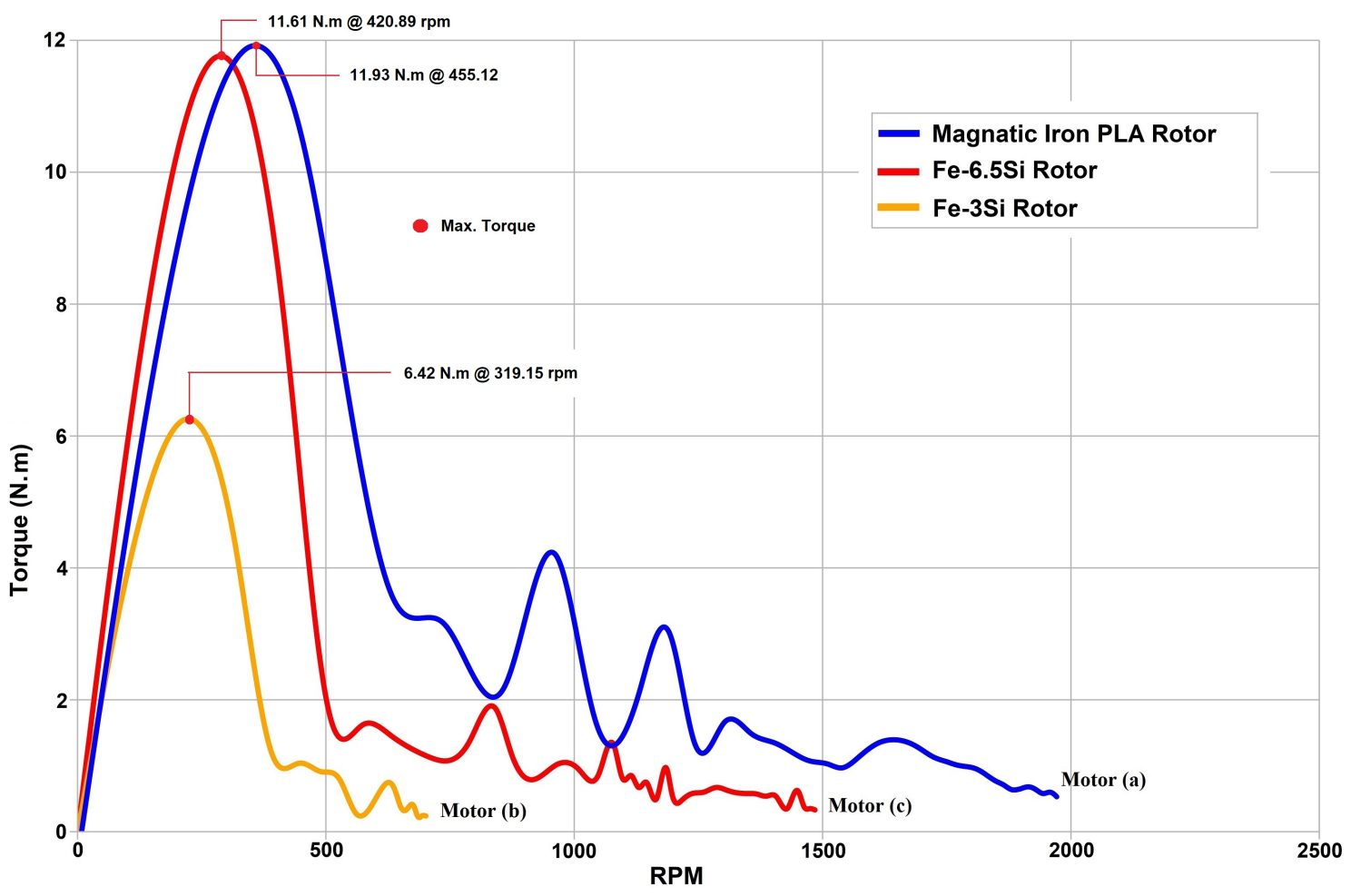

Figure 3.4: Torque Comparison

The magnatic iron PLA motor's torque reaches its maximum of $11.93 \mathrm{~N} . \mathrm{m}$ at a $455 \mathrm{rev} / \mathrm{min}$ speed. The Fe- $6.5 \mathrm{Si}$ motor's torque attains its highest torque 11.61 N.m at a speed of $420 \mathrm{rev} / \mathrm{min}$. Furthermore, the Fe-3Si motor's torque approaches its maximum of 6.42 N.m at only a $319 \mathrm{rev} / \mathrm{min}$ speed. The maximum RPM, power in, power out, efficincy, current,and voltage are also shown in the summary result Table ??.

\section{Max. Summary Results}

\begin{tabular}{cccccc}
\hline Description & Rpm & Spool Time & Torque $(\mathrm{N} . \mathrm{m})$ & Power Out $(\mathrm{W})$ & Voltage \\
\hline Magnatic Iron PLA & 2089.14 & @ $8.108872 \mathrm{sec}$ & $11.93 @ 455.12 \mathrm{rpm}$ & $0.55 @ 455.12 \mathrm{rpm}$ & $15.23 @ 2066.26 \mathrm{ppm}$ \\
\hline Fe-3.5Si Rotor & 738.77 & @6.177344 sec. & $11.61 @ 319.15 \mathrm{mpm}$ & $0.14 @ 319.15 \mathrm{rpm}$ & $17.7 @ 716.32 \mathrm{rpm}$ \\
\hline Fe-6.5Si Rotor & 1632.03 & @ $10.47399 \mathrm{sec}$ & $6.42 @ 420.89 \mathrm{mpm}$ & $0.33 @ 420.89 \mathrm{rpm}$ & $15.23 @ 1588.18 \mathrm{rpm}$ \\
\hline
\end{tabular}

Table 3.1: Summary Test Result (Max) 


\subsubsection{Conclusions.}

Many applications demand a high start-up torque. The D.C. motor, by its nature, has a high torque vs. falling speed characteristic, which allows it to deal with high starting torques and absorb unexpected rises in load efficiently. Hence, the speed of the motor adapts to the load. Accordingly, We can notice that the magnetic iron PLA motor has the highest start-up torque at the highest speeds than the other two silicon steel motors. This is because the Magnetic iron has no eddy current losses.

On the other hand, the Fe-3Si motor has the lowest torque and lowest speed. This section demonstrates that we can build the rotor using AM technology. The results showed that the motor rotor made using the magnetic iron PLA on an FDM machine has the best performance compared to the other two rotors built using silicon steel on CSAM. And therefore, the next step is to create the stator using AM technique. We will demonstrate the steps of how we manufactured the stator in the following section. Four stages were constructed to form the stator using different materials, different AM techniques and different geometries. Testing of all built stators was done using these rotors.

\subsection{Materials and Methods Used in the Manufacture of Stator Core}

A study was carried out to investigate the possibility of eliminating the PMs. Self-excited DC motor principles were selected for this, as electromagnetic induction is used in these types of motors to replace the PM. Since we can 3D-print the electromagnetic induction to build the rotor, we can use the same process to build the stator. The working principle of these motors is that the rotor and stator windings are connected in parallel. Consequently, the current in the stator and the rotor windings are not dependent on each other, as illustrated in Figure3.5. 


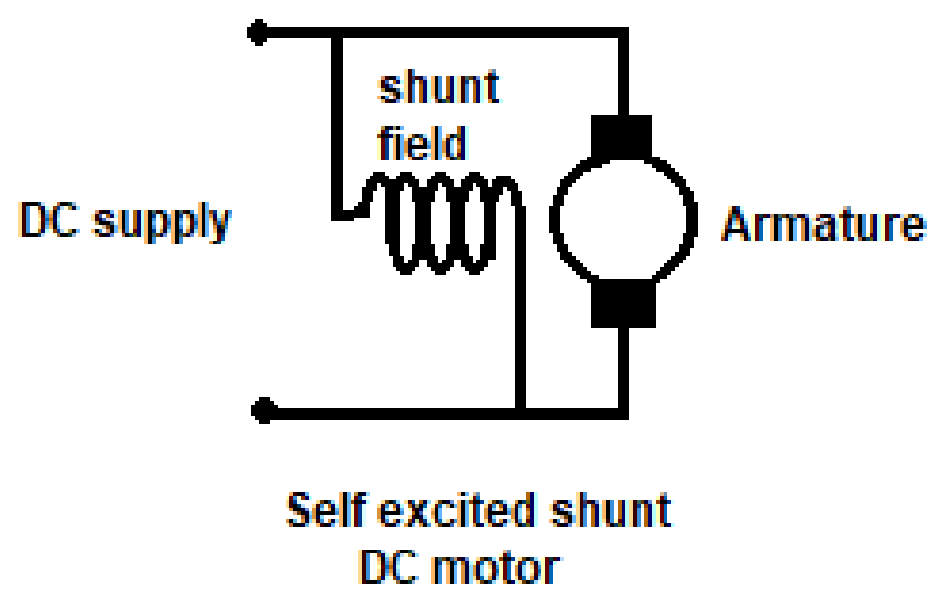

Figure 3.5: Self-excited shunt DC motor, Source: [142]

\subsubsection{Stator core made of Soft Iron (Fe $99.9 \%)$}

To apply the same principle as the Previously Fabricated motor, the four PMs were replaced by four soft iron Fe $99.9 \%$ rods with a diameter of $12.7 \mathrm{~mm}$ and a length of $35 \mathrm{~mm}$. The magnetic field of the electromagnet is intensified when the coil is wound on a soft iron core. Radially magnetized fast demagnetization can create an energetic field that is approximately $50 \mathrm{~K}$ times more intense than an air core, as stated by the manufacturer. The four poles were wound with copper coil $30 \mathrm{MAG}$, and the winding was done on a mini lathe machine (SHERLINE Model 4410), as shown in Figure 3.6. 


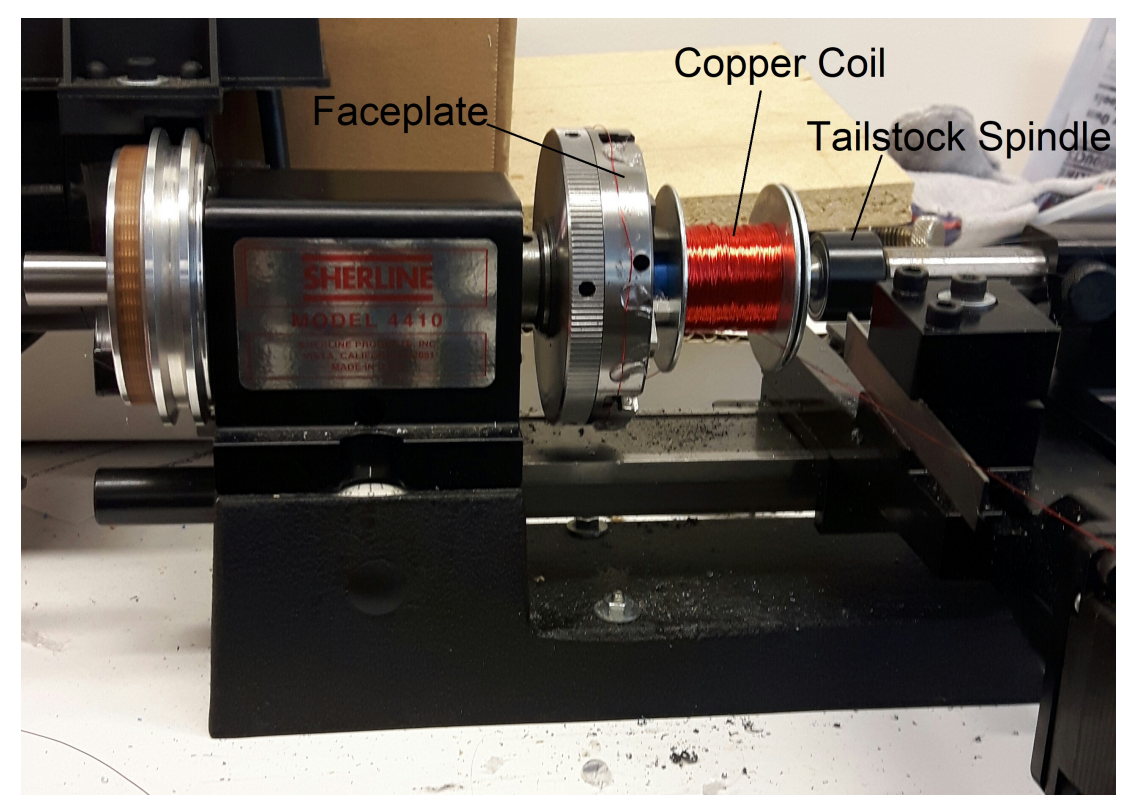

Figure 3.6: Copper Winding on Mini Lathe Machine

For the same number of windings, the outer diameters created were the same for all poles (approximately 32mm). The four poles were glued on L shaped steel brackets as in Figure 3.7. Although the poles were not 3D printed, they can be fabricated using SLS [143].

\subsubsection{Results and Discussion}

The motor was connected to an $18 \mathrm{~V}$ DC power supply. Measurements showed that the EMF was equal to 23 Gauss/pole with an air gap of $5 \mathrm{~mm}$, which is approximately 17 times more intense than the steel pole shoes. Testing showed that the two-pole shoes' attractive and resistive forces are powerful enough to rotate the armature. 


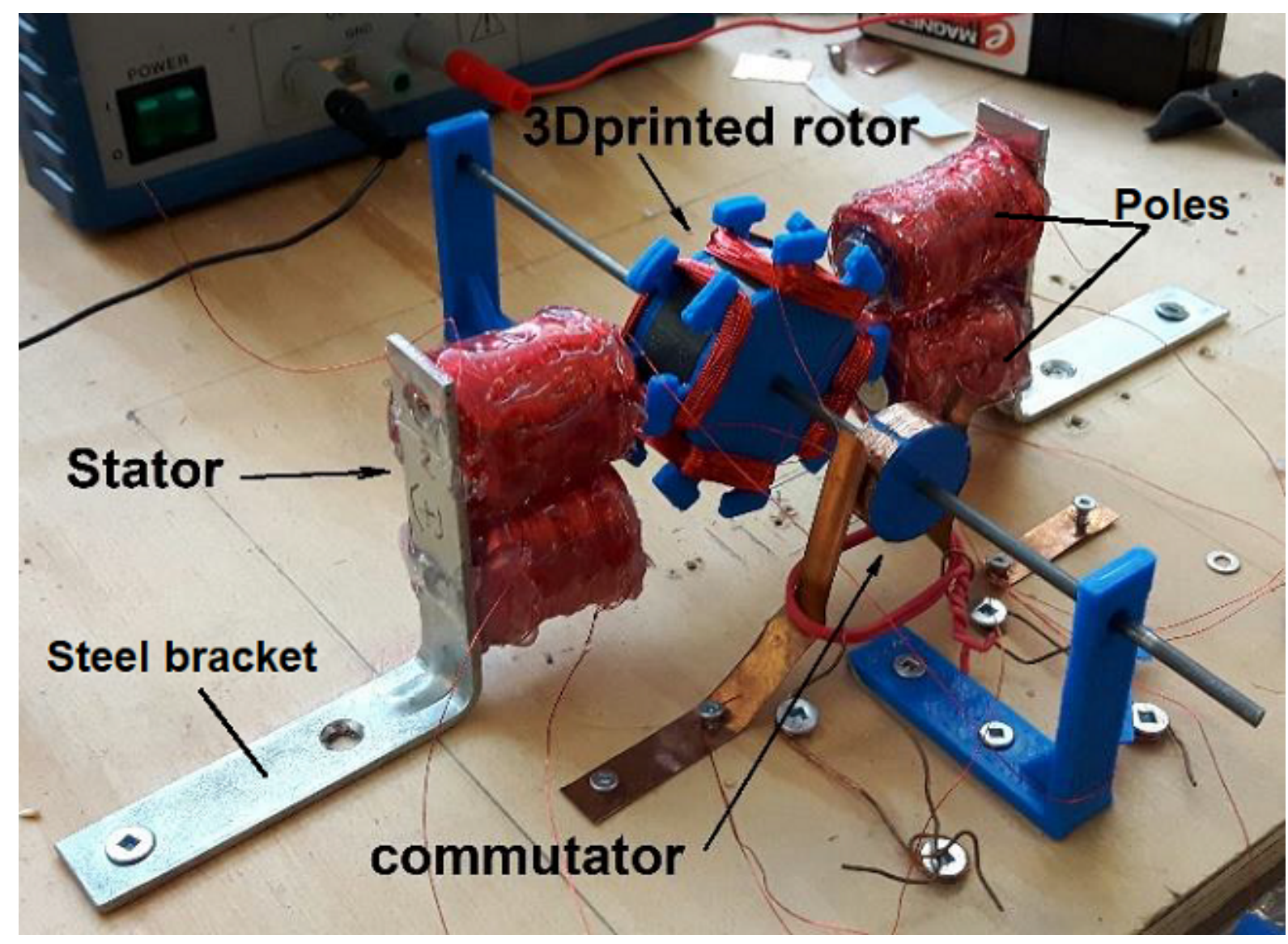

Figure 3.7: 3D printed Self-excited shunt DC motor.

\subsubsection{Stator Core Made of Maraging Steel (MS1)}

To build the prototype, the PMs are replaced by field coil wound on two steel 3D-printed pole shoes, as shown in Figure 3.8. The pole shoes' primary function is to spread the magnetic flux and prevent the copper coil from sliding. The two-pole shoes were built using EOS Maraging Steel MS1 powder on an EOS M290 machine that uses the Laser Powdeer Bed Fusion (LPBF) technique. EOS Maraging Steel is a tool steel powder designed for processing on EOS LPBF systems. This type of steel is characterized by having excellent mechanical properties and being easily heat-treatable using a simple thermal age-hardening process to obtain superior hardness and strength (see material data-sheet in Appendix A). The 3D-printing of the pole shoes was done by the ICAMP Innovation Center operated by Canadore College in North Bay, Ontario [144]. The chemical compositions of the EOS Maraging Steel MS1 are presented in Table 3.2. The two-pole shoes were wound with copper coil 26 MAG, 
and the number of turns per pole was 350 .

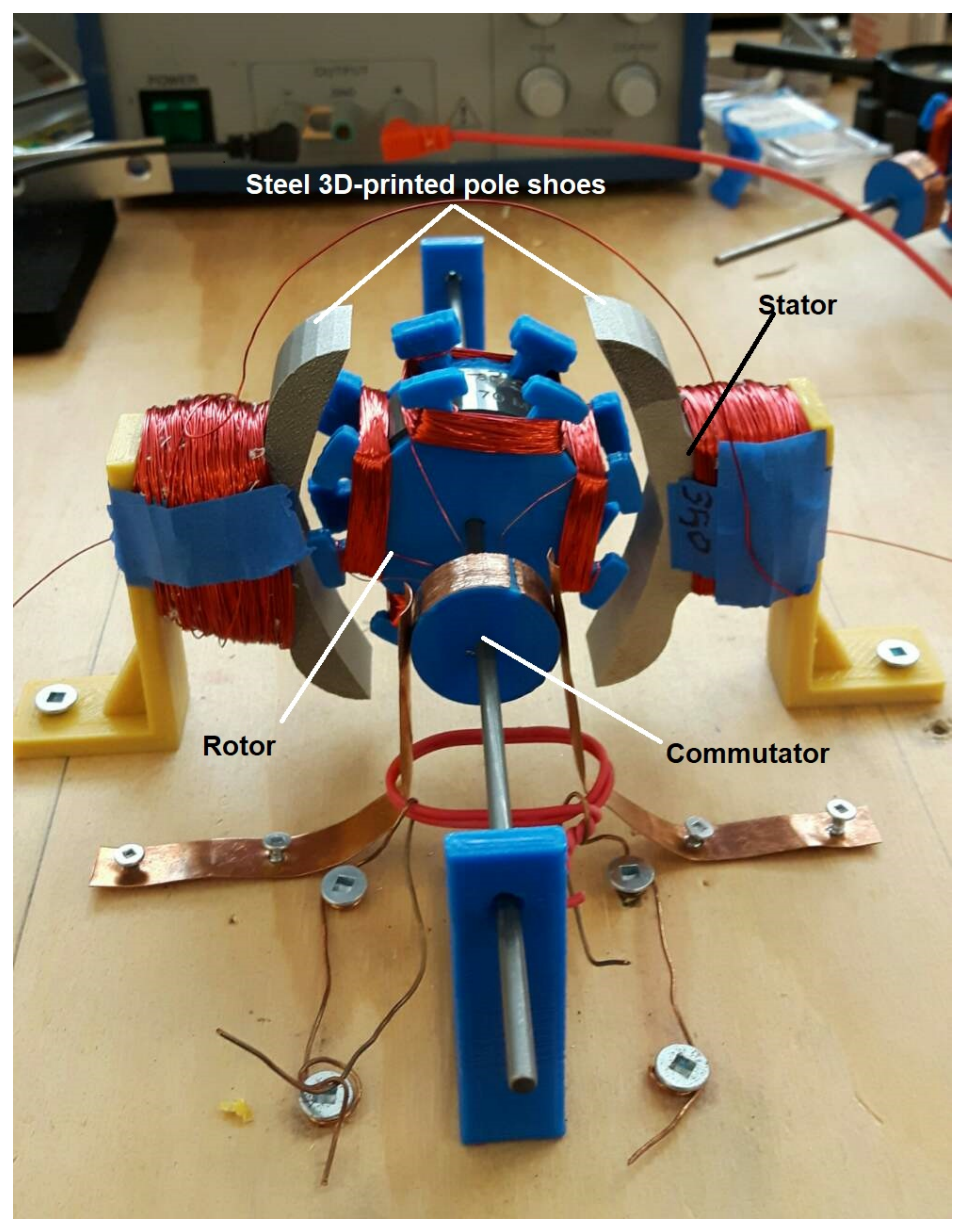

Figure 3.8: Self-excited DC motor (The 3D printed pole shoes made of steel)

Table 3.2: Chemical Composition of 3D Printed ASI 1008 Carbon Steel, Source: [144].

\begin{tabular}{|c|c|}
\hline Element & Content (\%) \\
\hline \hline $\mathrm{Fe}$ & balance \\
\hline $\mathrm{Ni}$ & $17-19 \mathrm{wt}-\%$ \\
\hline $\mathrm{Co}$ & $8.5-9.5 \mathrm{wt}-\%$ \\
\hline $\mathrm{Mo}$ & $4.5-5.2 \mathrm{wt}-\%$ \\
\hline $\mathrm{Ti}$ & $0.6-0.8 \mathrm{wt}-\%$ \\
\hline $\mathrm{Al}$ & $0.05-0.15 \mathrm{wt}-\%$ \\
\hline $\mathrm{Cr}, \mathrm{Cu}$ & each $0.5 \mathrm{wt}-\%$ \\
\hline $\mathrm{C}$ & $0.03 \mathrm{wt}-\%$ \\
\hline $\mathrm{Mn}, \mathrm{Si}$ & each $0.1 \mathrm{wt}-\%$ \\
\hline $\mathrm{P}, \mathrm{S}$ & each $0.01 \mathrm{wt}-\%$ \\
\hline
\end{tabular}




\subsubsection{Results and Discussion}

The motor was assembled with the same rotor built from Fe-3Si silicon steel particles, the same rotor that was built and tested in the first approach. The results showed that the coil heated up after a few seconds, and the produced EMF on the rotor was very weak (1.2 Gauss/pole) when connected to an $18 \mathrm{~V}$ DC power supply with a gap equal to $0.5 \mathrm{~cm}$ whcih is too low when compared to the soft iron stator that produces ( 23 Gauss/pole), which is too low when compared to the soft iron stator that produces ( 23 Gauss/pole), as can be seen in Table 3.3 which illustrates the EMF comparison between the soft iron stator and the 3D-printed steel stator. The weakness of the produced EMF on the steel stator is due to the low permeability of the 3D-printed steel. This result demonstrated the need to consider other materials with higher permeability, which will be investigated in the next section.

Table 3.3: Comparison between the 3D printed pole and the soft iron pole

\begin{tabular}{|c|c|c|c|}
\hline \multicolumn{2}{|c|}{} & EOS Maraging Steel MS1 Stator & Soft Iron Stator \\
\hline \hline Distance (cm) & Input DC Voltage & EMF (Gauss) & EMF (Gauss) \\
\hline 0 & $18 \mathrm{~V}$ & 3.1 & 41 \\
\hline 0.5 & $18 \mathrm{~V}$ & 1.2 & 23 \\
\hline 1 & $18 \mathrm{~V}$ & 1.1 & 14 \\
\hline 1.5 & $18 \mathrm{~V}$ & 1 & 8 \\
\hline 2 & $18 \mathrm{~V}$ & 0.9 & 5 \\
\hline
\end{tabular}

\subsubsection{Stator Core Made of Magnetic Iron PLA}

To overcome the 3D-printed steel prototype issue, the EOS Maraging Steel MS1 3D-printed was replaced by a magnetic iron open geometry stator made of PLA from Proto-Pasta (see printing parametrs of magnetic iron PLA filament in appendix B), as shown in Figure 3.9. The magnetic iron PLA from Proto-Pasta was chosen because it was successfully tested to build the rotors in the first approach. The 3D printed pole-shoes were wound with copper coil $30 \mathrm{MAG}$, and the number of turns for each pole was 900 . The testing was done using the same rotors that were built and tested previously.

The EMF was measured using an Arduino EMF detector. The results showed that the EMF of the stator was increased slightly compared to the 3D-printed steel prototype (1.6 Gauss/pole vs 1.2 respectively) when connected to the same power (i.e. 18V DC). However, this is far too weak to generate a stator field than the persistent magnetic fields produced by the rare earth magnet stator (51 Gauss/magnet).

This problem is due to the magnetic iron PLA not having the features of radially magnetized fast demagnetization compared to silicon steel or soft iron, even though the magnetic iron PLA showed an increase the intensity of a concentrated field slightly more than standard polylactic acid PLA. 


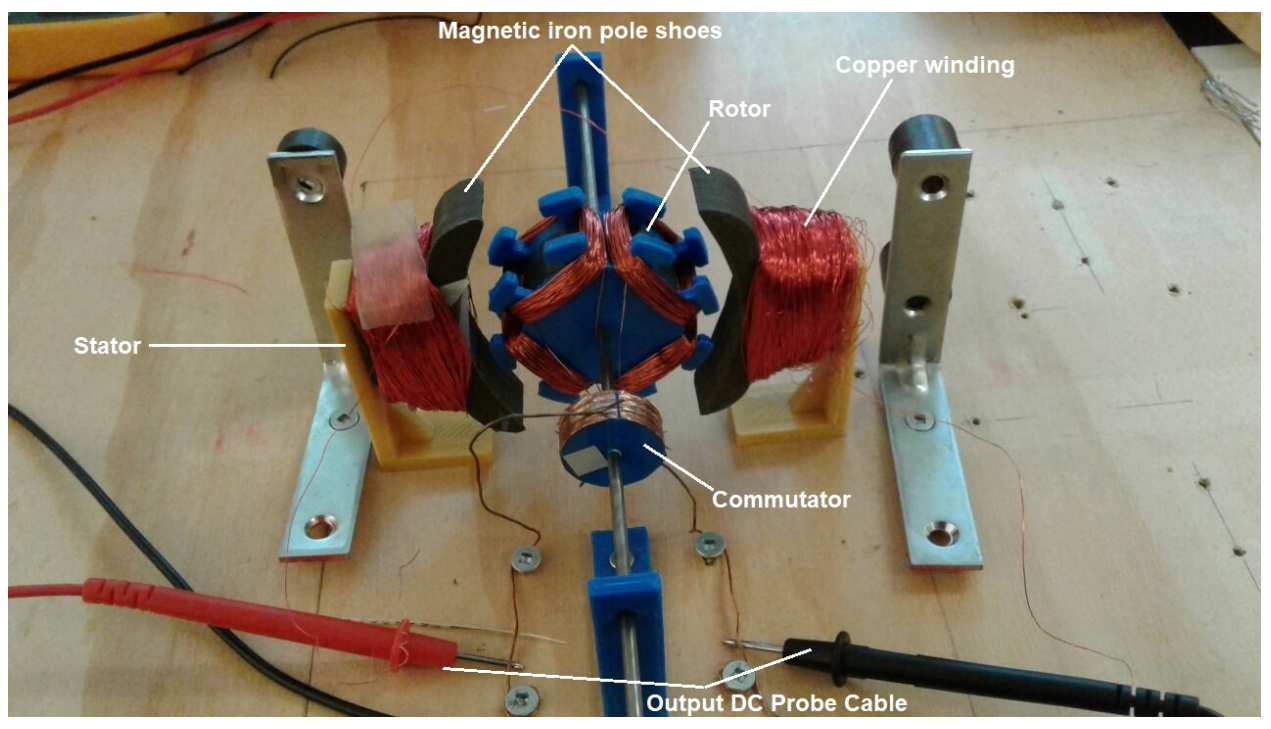

Figure 3.9: Open Geometry Self-excited DC Motor (Pole Shoes Made of Magnetic Iron PLA)

In order to amplify the electromagnet field on the stator, the closed geometry stator was replaced by an open geometry stator. This design has a hollow cylindrical shape in which the poles are fixed, the idea being that using a closed geometry will provide a low reluctance path for the magnetic flux. The stator was also 3D-printed with magnetic iron PLA, as shown in Figure 3.10. Both the rotor and stator magnets were wire wound, and the rotor winding was connected to the commutator through wire brushes using 24 MAG copper wire, which made the motor a series of wound self-excited DC. The commutator was a simple set of four thin contact copper sheets. The rotor was wire wound with 70 turns per pole, and the stator was wound with 100 turns per pole shoe. The main structure and commutator core were 3D-printed from ABS filament, which is more rigid than PLA filament. The only non-3D-printed parts were the copper wire coils and the steel motor shaft. 


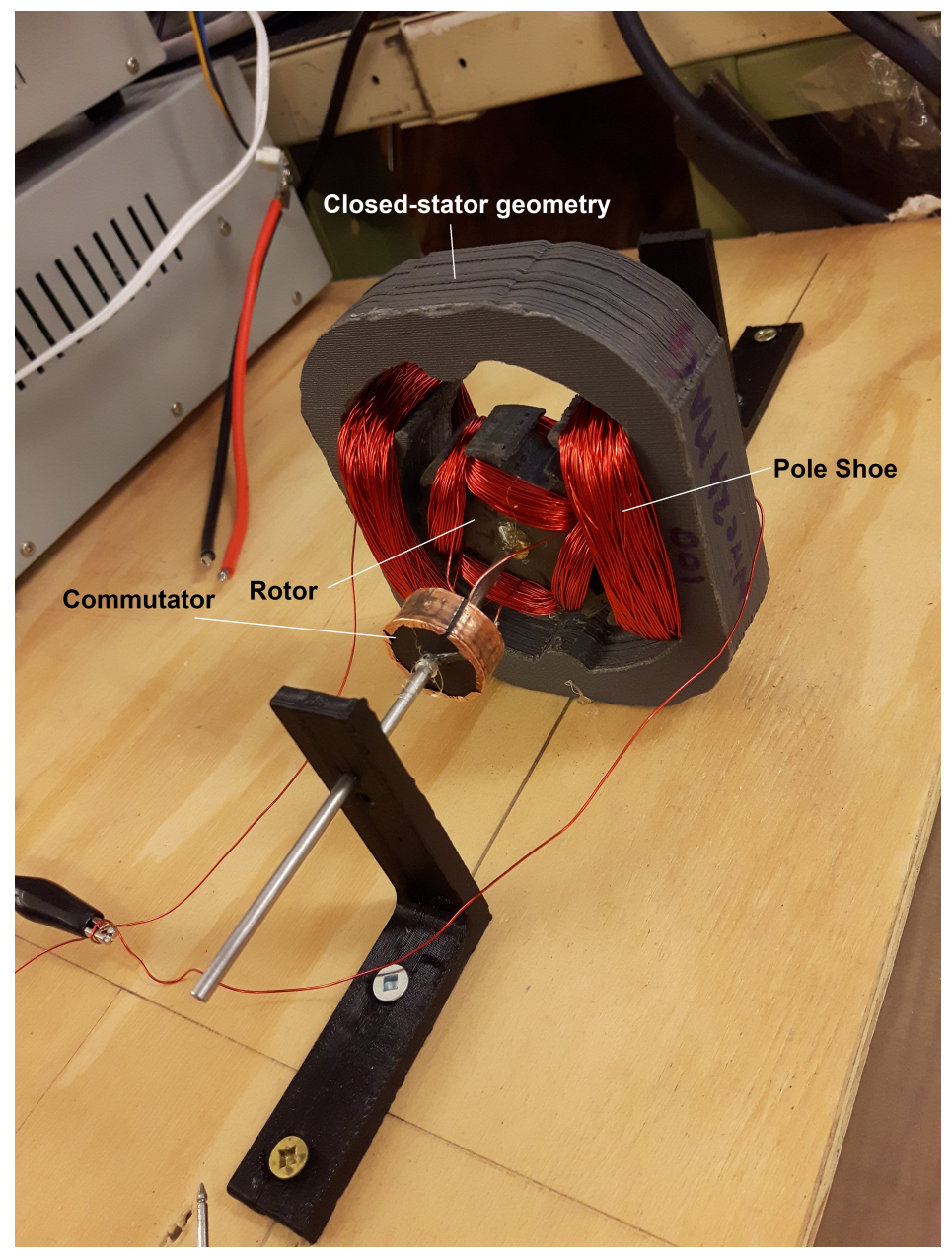

Figure 3.10: Closed Geometry Self-excited DC Motor (Pole Shoes Made of Magnetic Iron PLA)

\subsubsection{Results and Discussion}

The motor was connected to an $18 \mathrm{~V}$ DC power supply, and testing showed that the attractive and resistive forces of the two-pole shoes are strong enough to rotate the armature. Measurements showed that the EMF increased to 12 Gauss/pole using closed stator geometry, which is approximately eight times more intense than the open geometry, and was enough to run the motor successfully. This is because the closed stator geometry provided a low reluctance path for the magnetic flux compared to the open geometry. The lower the reluctance, the easier it is for magnetic flux to flow through the core material. Materials that are easily magnetized have a low reluctance and a high permeability, and non-magnetic materials have a high reluctance and a low permeability. 


\subsubsection{Conclusions.}

We demonstrated that 3D printed rotors successfully worked in the first stage when assembled with the conventionally constructed stator built using four soft iron Fe $99.9 \%$ rods. In the second stage, we learned that the two-pole shoes created by EOS Maraging Steel MS1 powder using the LPBF technique produce a very low EMF due to the low permeability of the 3D-printed steel. In the third stage, we considered magnetic iron PLA an alternative to building the pole shoes of the stator using open and closed geometries. We also demonstrated that the closed geometry produced more intense EMF than the open geometry. We also learned that the closed stator geometry provides a low reluctance path for the magnetic flux compared to the open geometry.

In the next chapter, Axial-flux motors were selected to develop the coils technique. This step is acknowledged in order to eliminate the use of conventional copper coils for the possibility of printing the winding on both sides of the rotor disks. 


\section{Chapter 4}

\section{Methodology: Fabricating and Testing of Axial-Flux DC (Pancake) Motor}

Pancake motor was only chosen to develop the coils technique to eliminate the copper coil winding on the rotor motor; hence axial-flux (pancake) motors that can have more than one rotor were selected. A Pancake DC brush motor structure is similar to a conventional motor, apart from its shape and size. Though pancake motors are more compact due to their thin rotors, they also consist of a rotor, commutator, stator, PMs, and winding [68], [145], [146]. This type was chosen to enable printing the winding on both sides of the rotor disks. The development of copper winding using AM techniques was established through 4 stages as per the following sections.

\subsection{Materials and Methods Used in the Manufacture of Motor Rotor Made of Copper Coil Winding Rotor}

The first step was building and testing an axial flux motor using copper coil winding, and the second step was to replace the copper wire and rotor core with a printed circuit board (PCB). The rotor, commutator and stator of this prototype were 3D-printed using ABS filament, as shown in Figure 4.1 (see printing parametrs of ABS filament in appendix B). The rotor was single-sided with four slots that form a four-pole, and each pole was wound with 95 turns of copper coil 30 MAG. The commutator was divided into four segments, each of which is attached to copper conductor tape and soldered by hand to the end of every winding coil. 


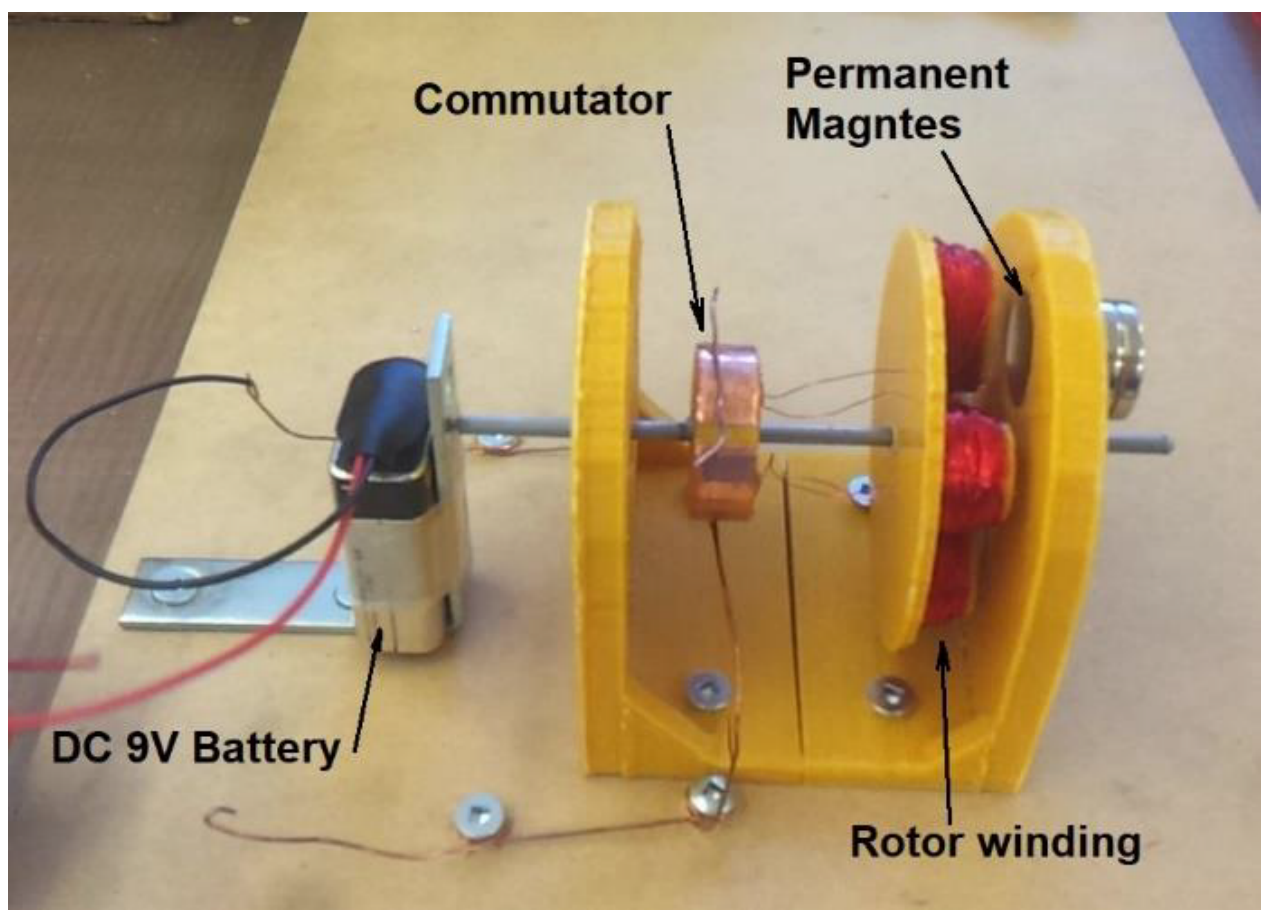

Figure 4.1: 3D printed DC brushed Pancake motor.

\subsubsection{Results and Discussion}

The motor was assembled and connected to a 9V DC battery, and the electricity ran through the two stationary brushes. A rotating magnetic field was generated according to the rotor winding directions, and the rotor aligned itself with the magnetic field of the two different PM poles, which led to a successful moving rotor. This result demonstrated that we could go forward to replace the copper coil winding with a printed circuit board (PCB) and then develop a technique to $3 \mathrm{D}$ print the winding on the rotor.

\subsection{Materials and Methods Used in the Manufacture of Motor Rotor Made of Printed Circuit Board (PCB)}

\subsubsection{Motor Rotor Made of Single-Sided Printed Circuit Board (PCB)}

The second prototype involved building the rotor without using the copper wire technique to reduce the complexity of 3D-printing the winding wires. A printed circuit motor was selected for this as it is likely to be 3D-printed, since 
the windings in this type of motor are etched on the printed board. Printed circuit motors have long been used in various applications, including high-speed tape readers, medical devices and industrial automation systems [147]. This prototype was fabricated using a rectangular shaped winding disc. A copper-clad single-sided PC board with a thickness of $1.6 \mathrm{~mm}$ and a diameter of $70 \mathrm{~mm}$ was used to create the first armature winding shown in Figure 4.2. The width of the winding trace is $1.02 \mathrm{~mm}$, and the clearance between the traces is approximately $1 \mathrm{~mm}$. The manufacturing process of this rotor was done by using the technique of producing PC boards.

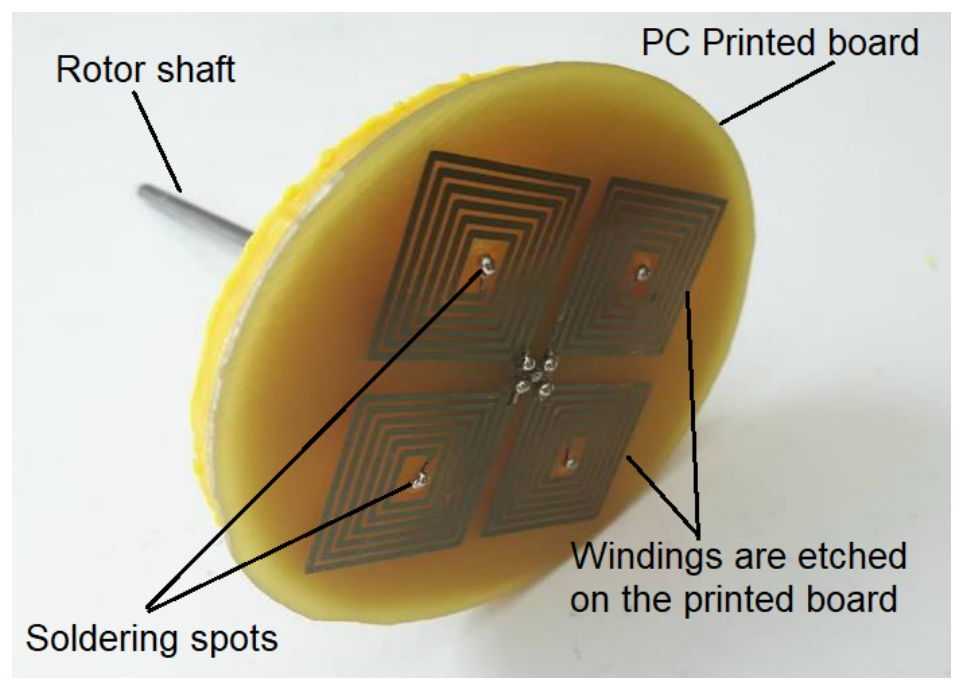

Figure 4.2: PCB rotor (Rectangularly shaped winding)

The process of prototyping both single-sided and double-sided circuits was conducted according to the following steps:

1. The Express PCB program was used to create the artwork for both the single and double layer boards, and a 600-dpi laser printer produced the PCB schematic drawing on transparency film 416-T.

2. Exposure kit 416-X from MG Chemicals was used with pre-sensitized boards that were exposed to UV light for approximately 10 minutes from a distance of $12.7 \mathrm{~cm}$.

3. The development process was used to remove any photoresist exposed by the UV light. This was done by immersing the board into a solution of one part M.G. Developer (Cat. No. 418) to ten parts water. The exposed resist was removed from the board after one to two minutes, and the only resist remained was the covered schematic of the circuit, as shown in Figure 4.3. 
4. The board etching process was performed using an etching kit (Cat. No.416-ES) and ferric chloride etchant (Cat No. 415) from M.G. Chemicals, according to the manufacturer's instruction [148]. The process involves immersing the board in the ferric chloride, where the air bubbles created by the etching kit help remove the unwanted copper [68].

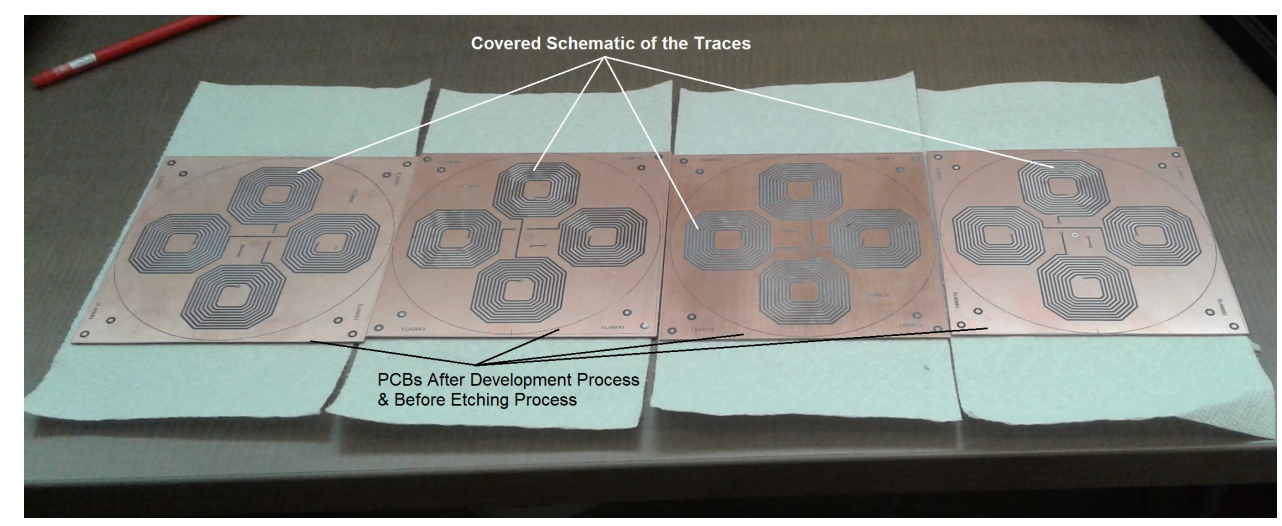

Figure 4.3: PCB Schematic Drawing

The rotor, commutator and stator were all 3D-printed using ABS filament. After etching the board and creating the artwork, the board was cut to the shape of the rotor using a shear cutter and attached to the 3D-printed rotor. The commutator was divided into four segments. Each one was attached to the copper conductor tape and soldered by hand to the end of every wound trace. The motor was then assembled and connected to a 9V DC battery, as shown in Figure 4.4. Testing the motor with an air gap of approximately $2 \mathrm{~mm}$ showed that it produced minimal electromagnetic force, and was unable to either attract or repel the PMs. This was due to the rotor's space limitations, where only five turns were printed on a single-sided board. 


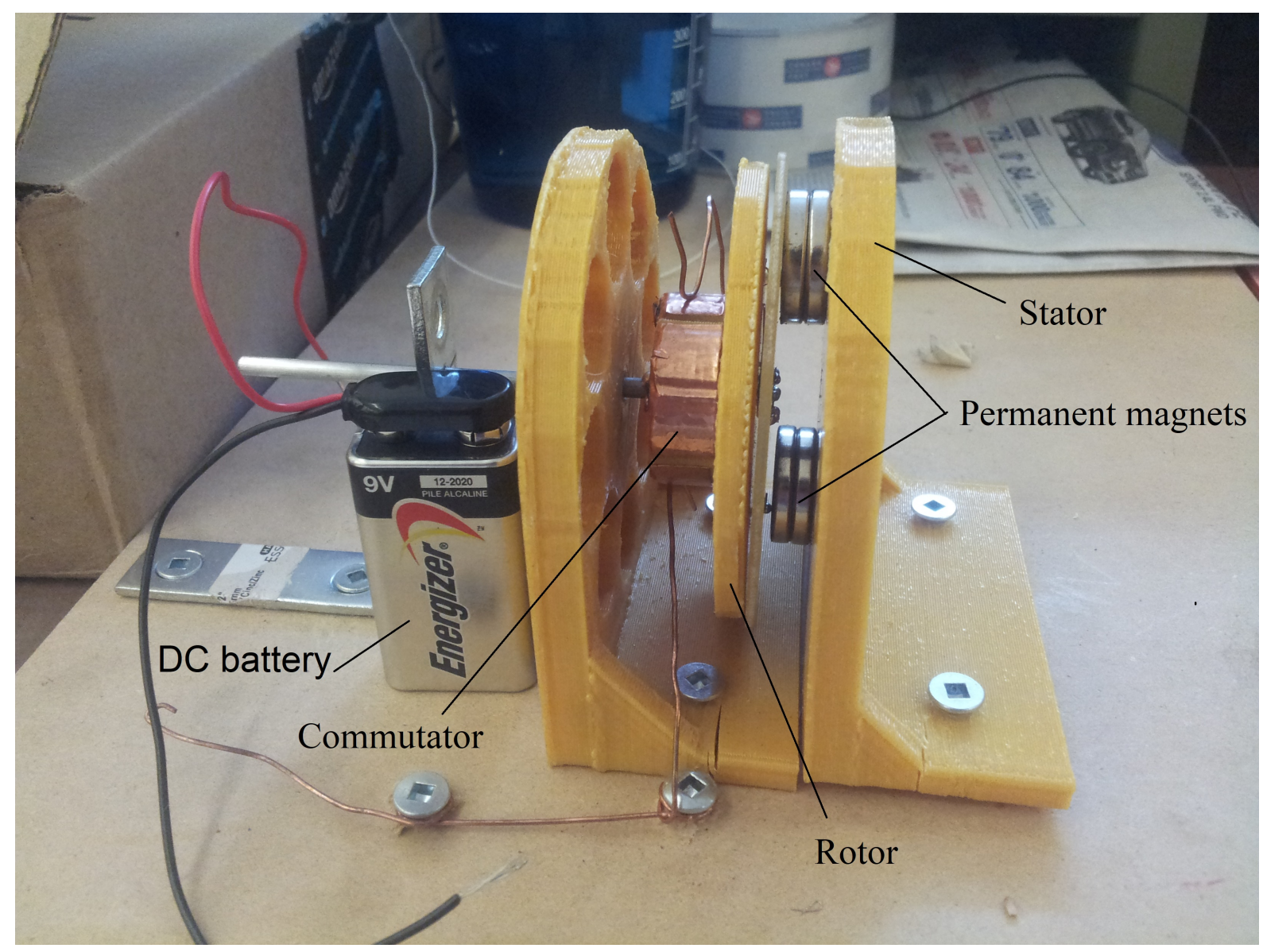

Figure 4.4: Printed Circuit Motor (single- sided board)

\subsubsection{Motor Rotor Made of Double-Sided Printed Circuit Board (PCB)}

To overcome the issue encountered in the second prototype, a copper-clad double-sided PC board with a thickness of $1.6 \mathrm{~mm}$ and a diameter of $150 \mathrm{~mm}$ was used to create the armature winding, as shown in Figure 4.5. The reason for using the double-sided board and doubling the diameter of the rotor was to increase the number of turns by increasing the winding space, and to have winding on both sides. The rectangularly shaped winding was replaced by an invented octagon-shaped winding to increase the number of turns, which led to an increase in the electromagnetic force. This accommodated nine turns per winding side, 18 in total, with $1.02 \mathrm{~mm}$ wide traces and winding clearance between them of approximately $1 \mathrm{~mm}$. With the two rare earth circular magnets buried in the inner stator core, testing showed that the rotor produces enough electromagnetic force to run the motor. 


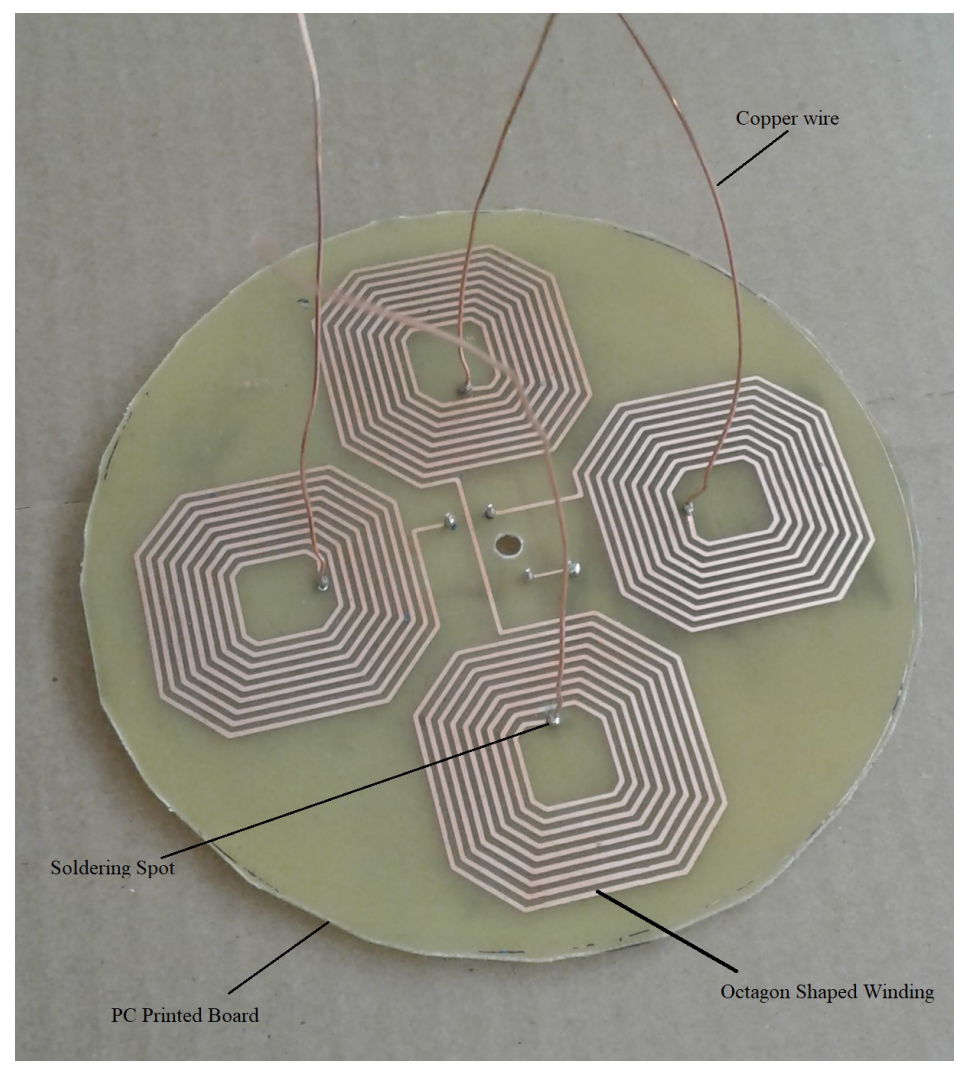

Figure 4.5: Single PCB rotor (octagon shaped winding)

Another motor was built with the preceding procedures with the objective of increasing the number of windings. This was accomplished with two connected double-sided PC boards separated by thin 3D-printed layers made of ABS filament, as shown in Figure 4.6, (see printing parametrs of ABS filament in appendix B).

Building two prototypes of PCB pancake motors showed that the single disk rotor produced enough electromagnetic force to attract or repel the PMs with the motor rotating at $500 \mathrm{RPM}$ when connected to $12 \mathrm{~V}$ DC power. The double disk rotor showed an increase in speed up to 700 RPM under the same conditions (i.e. the same voltage). The results demonstrated that $3 \mathrm{D}$-print electric motor could be achieved by using different techniques. 


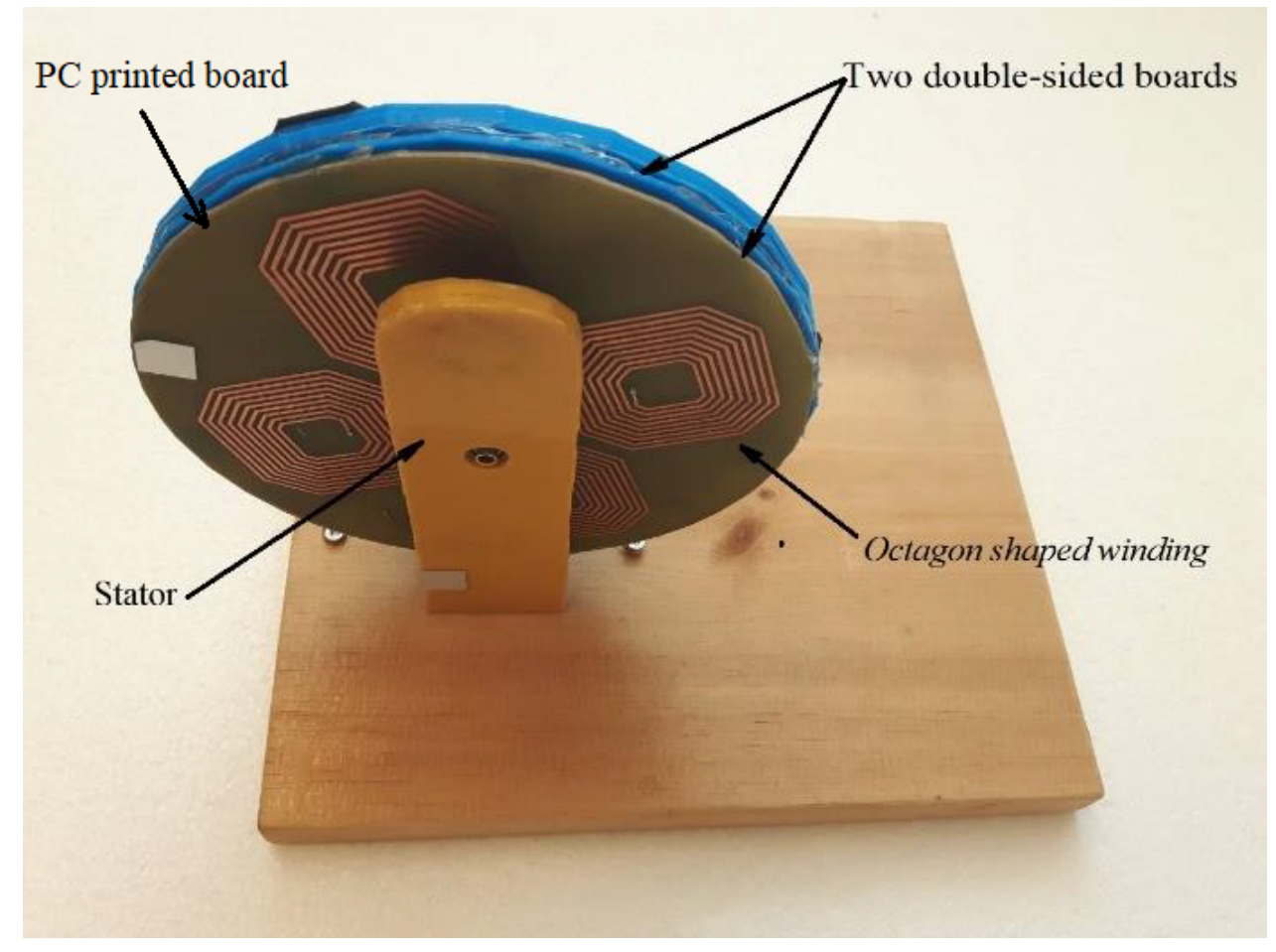

Figure 4.6: Multi-layer PCB rotor (octagon shaped winding)

\subsubsection{Results and Discussion}

The motor made of a single-sided printed circuit board (PCB) in the first prototype showed that it produced minimal electromagnetic force and could not either attract or repel the PMs. This result was due to the rotor's space limitations, where only five turns per pole were printed on a single-sided board. In the second prototype, when the diameter of the rotor was increased to accommodate more turns using a double-sided printed circuit board; where the rotor accommodated 18 turns per pole. As a result, the motor test showed that the printed rotor produces enough EMF to run the motor with a speed of $500 \mathrm{rpm}$ when connected to $12 \mathrm{~V}$ DC power. In the third prototype, when the duple-sided disk rotor motor is connected to $12 \mathrm{~V} \mathrm{DC} \mathrm{power,} \mathrm{it} \mathrm{rotates} \mathrm{with} \mathrm{a} \mathrm{slightly}$ higher speed equal to $700 \mathrm{RPM}$. This result proves that we can have as many layers as we want to improve motor performance. Even though manufacturing the PCB is called the printing process, they can't be considered 3D printing processes. Therefore, the next step is to examine how to replace the same procedure with $3 \mathrm{D}$ printing techniques. 


\subsection{Materials and Methods Used in the Manufacture of Motor Rotor Made of Conductive Copper Filament}

To eliminate the use of copper winding or PCB on the rotor motor. Another experiment was conducted to investigate using conductive copper filament (Electrifi by Multi3D), which is the only 3D-printing filament on the market considered to be conductive; the manufacturer states it has a resistivity of $0.006 \Omega \mathrm{cm}$. The challenge here was determining how to print two materials with a single head printer. To accomplish this, 3D-printing of the substrate was done first with magnetic iron PLA filaments, then cooled, and the printing bed was leveled according to the substrate, rather than the bed. After repeatably attempting to print traces using Electrifi, a print/no print issue was encountered since the extruder system of the 3D-printer (Anet A8) could not push the filament with sufficient pressure due to its flexibility, and this led to the nozzle becoming clogged. To deal with this, a series of attempts were conducted until the printing worked adequately. The issue was solved using the following steps:

1. The tension from the lever and the hobbed gear was too high to handle semi-flexible filaments, as the gear teeth would bite too deeply into the filament and scrape it off. The tension was reduced until the ideal pressure was determined.

2. The print temperature for Electrifi is lower than most other filaments (e.g. PLA, ABS), which meant the previous residual filament would cause clogging very quickly if the nozzle was not well cleaned. A spare, clean nozzle was kept on hand to address this problem.

3. Lowering the print speed to $15 \mathrm{~mm} / \mathrm{s}$ was found ideal for a $0.5 \mathrm{~mm}$ standard brass nozzle.

4. The distance between the nozzle tip and the print bed must be greater than $0.2 \mathrm{~mm}$ to ensure the printed first layer is not overly compressed.

It was determined that the appropirate printer setup when using this type of filament are as shown in Table: 4.1 
Table 4.1: Perfect printer setup for Electrifi filament.

\begin{tabular}{|l|l|}
\hline Nozzle type & Standard brass \\
\hline \hline Nozzle diameter & $0.4 \mathrm{~mm}$ \\
\hline Extruder temperature & 160 -degrees Celsius \\
\hline Bed temperature & Room temperature \\
\hline Layer thickness & $0.25 \mathrm{~mm}$ \\
\hline Print speed & $15 \mathrm{~mm} / \mathrm{s}$ \\
\hline Layer height & $0.25 \mathrm{~mm}$ \\
\hline Print bed & Blue tape \\
\hline Initial layer height & $0.25 \mathrm{~mm}$ \\
\hline
\end{tabular}

\subsubsection{Results and Discussion}

After successful trace printing on a printed rotor, as shown in Figure 4.7, (see printing parametrs of conductive copper filament in appendix B), the motor was connected to a low voltage DC power supply to test the conductivity and electromagnetic force. An incremental voltage increase to $3.4 \mathrm{~V}$ resulted in very high contact resistance between the probes and traces, and the hot spots heated up when the resistance reached $17 \Omega$ at $200 \mathrm{~mA}$. This verifies that the filament cannot be used to build our electric motors, and is suitable only for low current applications.

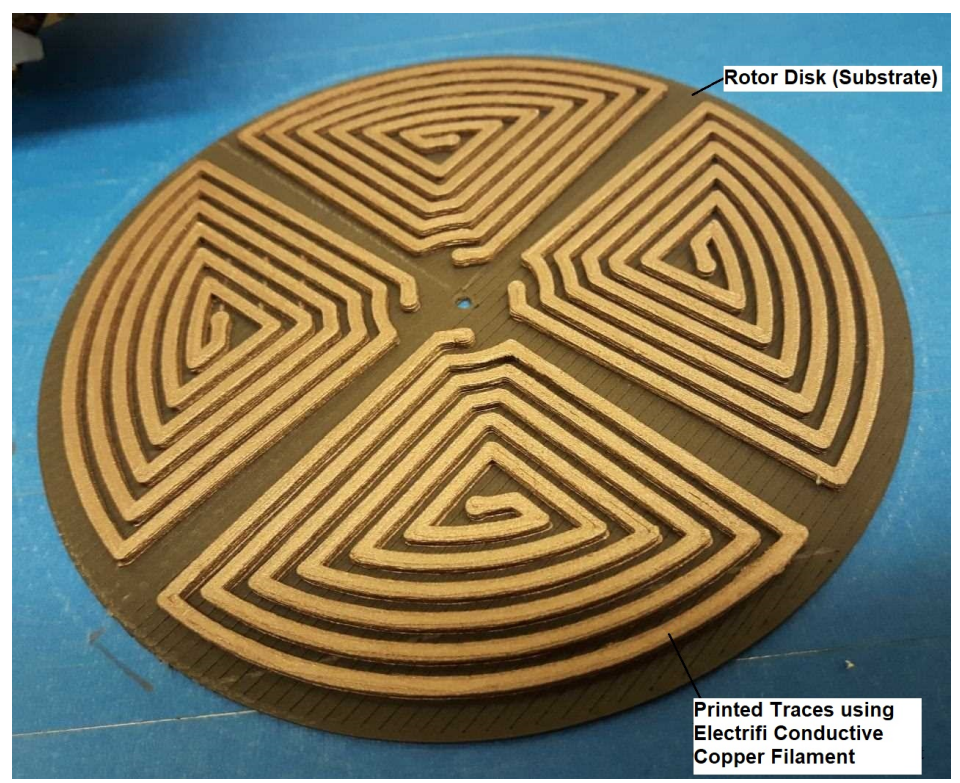

Figure 4.7: Fully 3D printed Armature made out of conductive copper Filament

The conclusion for the copper conductive filament is that it cannot be used for high power applications, only for low power like a small, efficient LED. The properties of copper conductive filament and its implementation 
was measured using a simple Ohmmeter and the following formula:

$V=R \times I$

Or: $P=R \times I^{2}$

Where:

$P=$ The electric power in watt $(\mathrm{W})$.

$V=$ The voltage in volts $(\mathrm{V})$.

$I=$ The current in amps (A).

$R=$ The resistance in ohms $(\Omega)$.

\subsection{Materials and Methods Used in the Manufacture of Motor Rotor Made Using Laminated Object Manufacturing Like Process}

After searching for an alternative to 3D-printing the whole rotor and eliminating the use of copper winding or PC printed boards, a new prototype was constructed using the laminated object manufacturing technique (LOM), a 3D-printing process developed by California-based Helisys Inc. (now Cubic technologies). During the LOM process, layers of copper or paper are fused/laminated together using heat and pressure, then cut into the required configuration with a computer-controlled blade or laser [53], as illustrated in Figure 4.8. Accordingly, we could use the LOM technique to print the winding traces on a 3D printed substrate. The benefit of this process is to form the winding so that we could have an entirely $3 \mathrm{D}$ printed rotor. If succeeded, the rotor could also be created of multi-layers to increase the performance of the motor. 


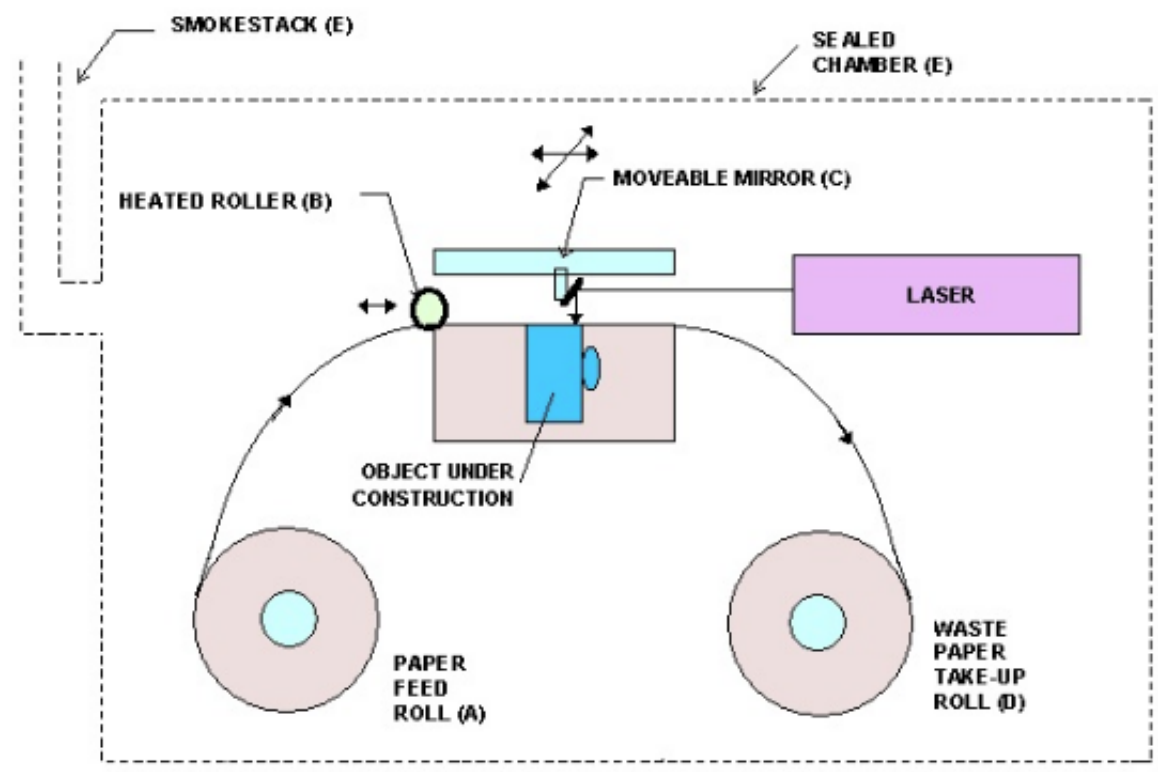

Figure 4.8: Laminated Object Manufacturing (LOM), Source: [53]

Though this machine is not available at Carleton University, the same idea can be applied using simple tools. The Circuit Explore Air 2 machine shown in Figure 4.9 was tailored to perform the LOM process. The winding traces are drawn on the machine software, and after feeding the copper tape to the machine, it cuts the copper into the required shape, and the unwanted material can then be easily removed. The copper winding traces can then be taped onto the 3D-printed rotor. 


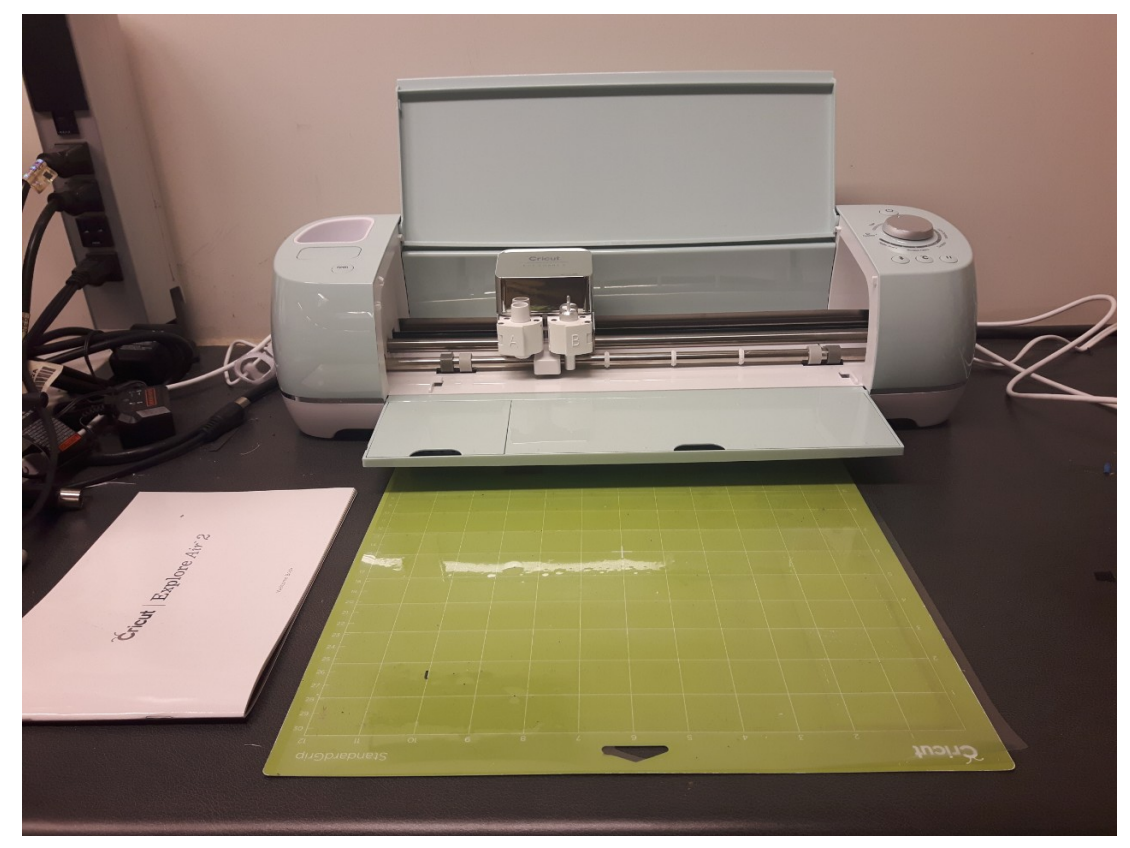

Figure 4.9: Circuit Explore Air 2 machine

Accordingly, the new prototype was fabricated in the previously mentioned idea. The rotor and the commutator were designed to be attached as one piece. Both rotor and commutator were 3D printed by using magnetic iron PLA, Rotor diameter was $150 \mathrm{~mm}$ with a thickness of $2 \mathrm{~mm}$, and the commutator diameter was $25 \mathrm{~mm}$. The Circuit Explore Air 2 machine was used to cut the shape of the armature winding and taped on both sides to create a double-sided rotor, as shown in Figure 4.10. 


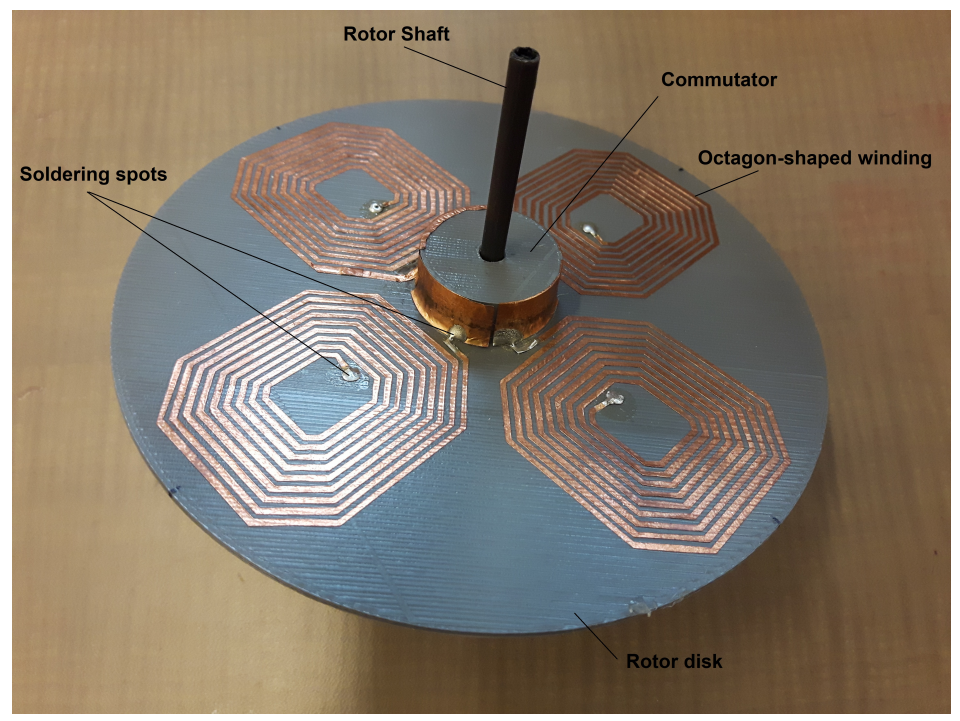

Figure 4.10: Copper Tape Winding Traces on 3D Printed Rotor

Four poles were created with nine windings per side, so 18 turns in total. The width of the winding trace is 1 $\mathrm{mm}$, the clearance between traces is approximately $1 \mathrm{~mm}$, and the thickness of the copper foil is $0.0762 \mathrm{~mm}$. The commutator was divided into four segments, and each were attached to a copper conductor tape and soldered by hand to the end of each winding coil. 


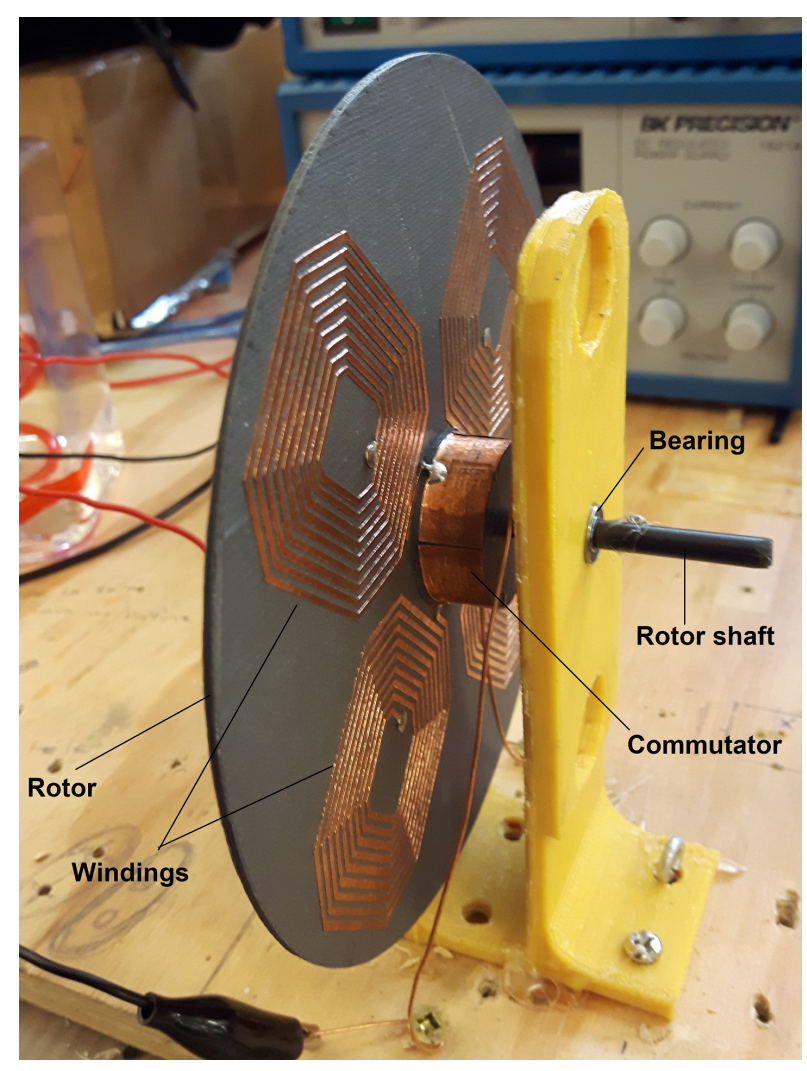

Figure 4.11: 3D Printed Pancake Motor

\subsubsection{Results and Discussion}

The motor was assembled with a 3D-printed open stator, as shown in Figure 4.11, and the two rare-earth circular magnets were buried in the core of the inner stator. When connected to $9 \mathrm{~V} \mathrm{DC}$, the rotor produces electromagnetic force very similar to the previous PCB constructed motor, and the magnetic flux magnitude allows the motor to run successfully. Thus, it has now been demonstrated that a 3D-printed pattern can replace the copper wire. The following section's motivation is to use what we have learned to build a traditional electric motor that was partially 3D printed in the previous approaches. The idea is to apply the same concept of LOM processes assuming that we could 3D print the copper foil on a traditional rotor using the LOM-like technique.

\subsection{Materials and Methods Used in the Manufacture of Fully 3D Printed Radial-Flux DC Motor}

As discussed, the FDM and LOM techniques were applied to build the 3D-printed pancake motor. This prototype focuses on using the same techniques to complete a traditional electric motor that was partially 3D- 
printed in the previous approaches. The traditional electric motor is the most fundamental design from which all other motors are derived [149]. This approach will 3D-print all parts of the motor, including the PMs. It is designed with open geometry to provide easy access to every part and consists of the 3D-printed motor base, the rotor core, stator brackets, commutator, axle, bearings, nuts and bolts, as shown in Figure 4.12.

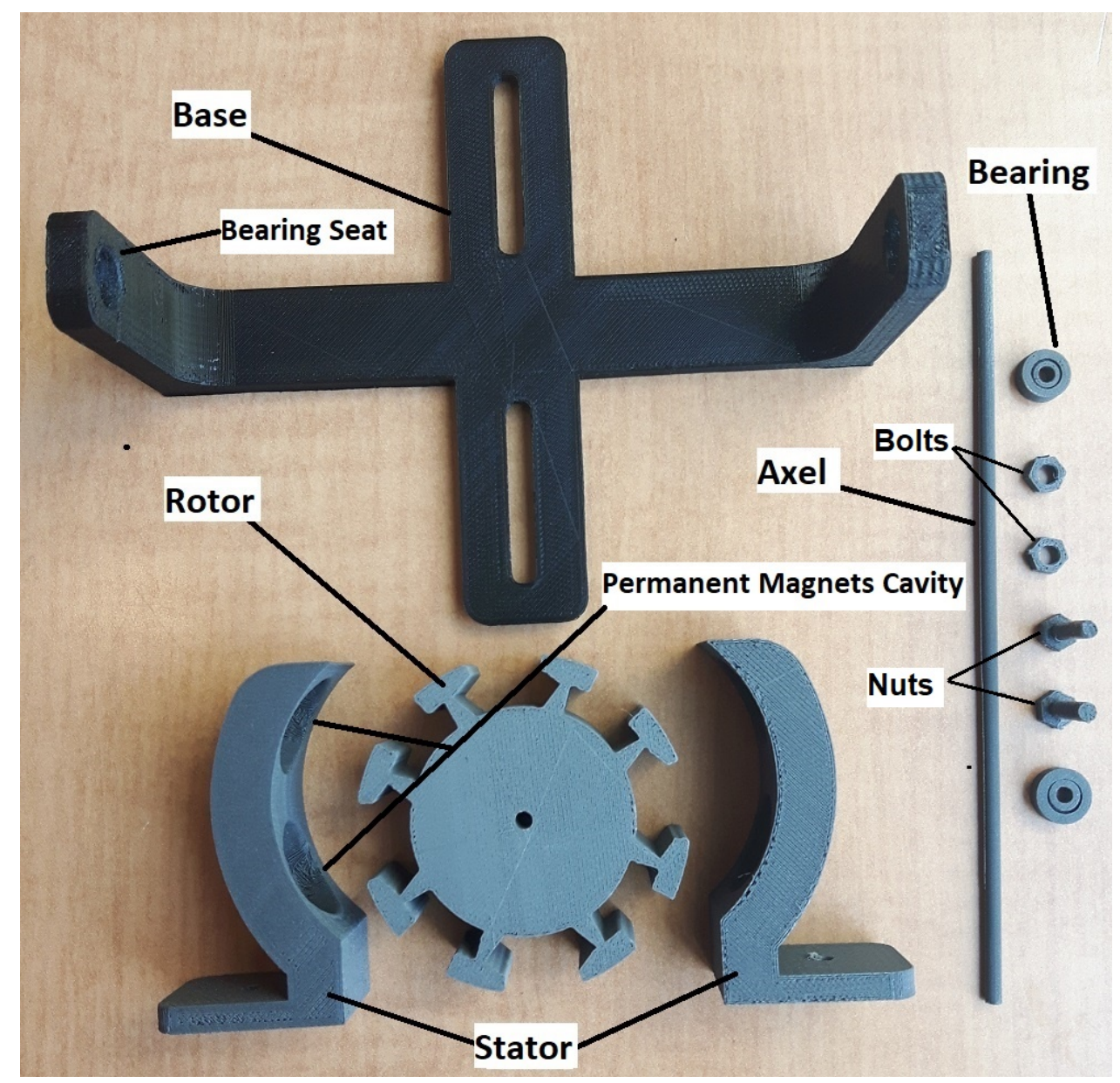

Figure 4.12: 3D printed parts of the DC Motor

Using the Anet A8 3D printer, all parts were made of magnetic iron PLA (see printing parametrs of magnetic iron PLA filament in appendix B), except for the motor base made of regular PLA (see printing parametrs of PLA filament in appendix B). Four 3D-printed PMs were built from NdFeB by the Oak Ridge National Laboratory (ORNL) [94]. Oak Ridge National Labs (ORNL) utilizes additive manufacturing to manufacture 3D printed permanent magnets using the Big Area Additive Manufacturing (BAAM) machine. Instead of using filament melting, which is too slow to keep up with the deposition rates needed to create large magnets. ORNL directly extrudes pellets of over 5 inches in diameter at high speed to fabricated isotropic, near-net-shape, neodymium- 
iron-boron $(\mathrm{NdFeB})$ bonded magnets [92]. These magnets shown in Figure 4.13 were buried in the inner stator core.

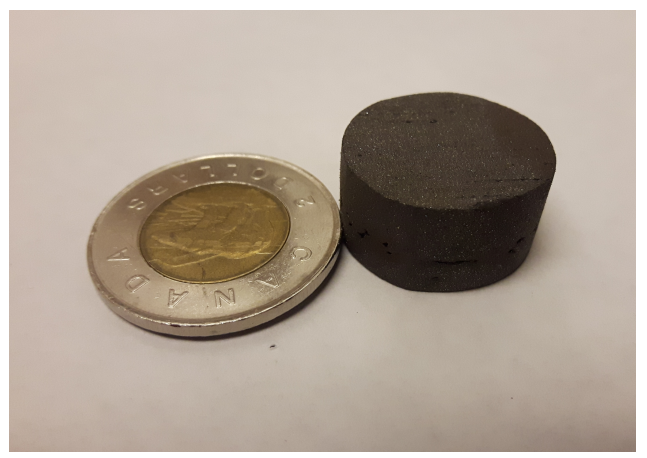

Figure 4.13: 3D Printed Permanent Magnet

Eight equally spaced slots were formed at the outer circumference of the rotor core and used to wind the copper foil tape. Four poles were wound with copper tape (SPARKFUN \#SF-PRT-11081) with a thickness of 152 microns and an approximate width of $5 \mathrm{~mm}$, with 17 turns per pole. The copper tape was insulated using silicone modified conformal coating before it was wound by hand, though it could also be fabricated with LOM. Four sets of the 152 micron thick contact copper sheets were attached to the commutator; the conductive glue side of the copper foil made it easy to attach the ends to the commutator copper tapes without soldering. Both the rotor core and the commutator were attached to the printed axle, which was $60 \mathrm{~mm}$ long with a diameter of $3.175 \mathrm{~mm}$, as shown in Figure 4.14. 


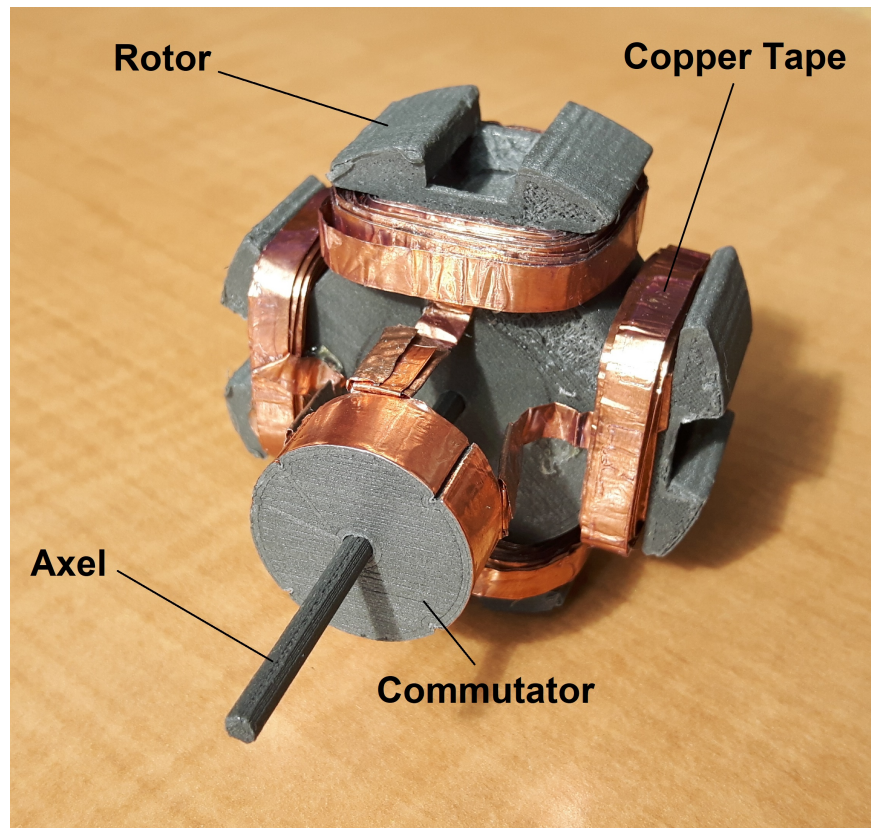

Figure 4.14: Rotor Assembly

\subsubsection{Results and Discussion}

The rotor was assembled with the motor base and the stator, and two copper wires of $20 \mathrm{GA}$ were used to form the brushes, as shown in Figure 4.15. To test the motor, it was attached to an 18V DC power source connected to the rotor winding through the commutator and brushes. The two brushes contact the copper conductor tapes to reverse the flow of current in the rotor, and this results in a successful rotor movement. 


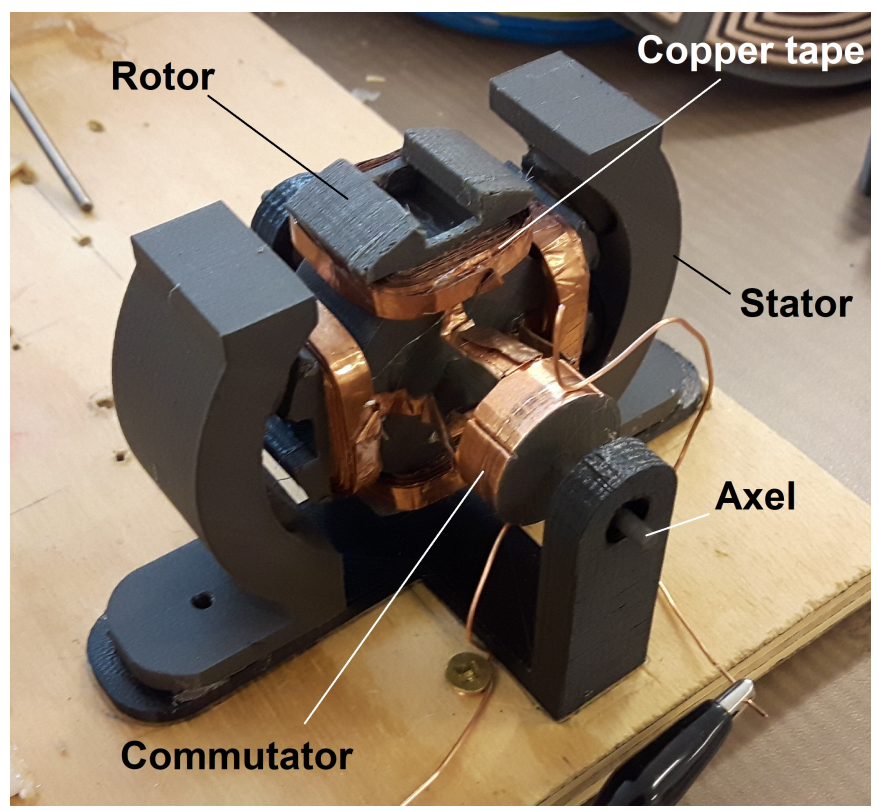

Figure 4.15: Fully 3D Printed Motor

\subsection{Conclusions.}

This chapter aimed to identify whether we can develop the coils technique to eliminate the copper coil winding on the rotor motor. Based on the analysis of the built prototypes, it can be concluded that the magnetic flux magnitude resulted from the motor rotors made using a LOM-like process indicated the potential of a 3D-printed pattern to replace the copper wire using the LOM technique. Due to the limited access to such a machine, further investigation has to be conducted to confirm this process. The difference in the architecture of all the previous 3D printed motors compared to the off-the-shelf motors does not allow for a direct comparison. Therefore, in next chapter, we used reverse engineering to create a $3 \mathrm{D}$ printed motor similar to the traditional motor to validate performance and benchmark. 


\section{Chapter 5}

\section{Validate Performance and Benchmarking}

This section aims to produce a prototype that will be employed for validating the electromechanical performance experimentally and provide a direct comparison with the classically manufactured counterparts. The difference in the architecture of all the previous 3D printed motors compared to the off-the-shelf motors does not allow for a direct comparison. Therefore, in this section, we used Reverse Engineering (RE) process to create a 3D printed motor very similar to the traditional motor in terms of geometry, size, the number of poles, copper coil gage, number of turns, and type of permanent magnets to validate performance and to benchmark. Building this motor involved the following stages:

1. Selecting the appropriate product from the market,

2. Motor disassembly,

3. Measuring of all parts and data collecting,

4. Creation of 3D CAD models,

5. Manufacturing,

6. Final product testing, comparison and evaluation.

Testing was done using a universal benchtop inertia motor dynamometer (dyno). The used dyno can measure the rpm, $\mathrm{Kv}$ (rpm/voltage), voltage, current draw, power, and torque output of the commercial and the 3D printed motors. The 3D-printable magnetic PLA from Proto-Pasta has been used to 3D print the motor parts. The material is not a magnet as it's called, but a magnet attracts it, and it also can't be permanently magnetized. With its properties, the material could be beneficial to 3d-print special-shaped cores for electromagnetic coils such as an electric motor. Our previous tests with this material's magnetic behaviour show that the magnetic PLA is much less magnetic than common things made of steel. On the positive side, the material is not conductive: therefore, there is no danger to create short-cuts when winding the coils, and there are no eddy currents. 


\subsection{Motor Design}

The design specification for the brushed DC motor was established based on commercially available DC motors. A small DC permanent magnet motor showed the figure 5.1 was chosen to be reverse-engineered. The motor is a $20 \mathrm{~T}$ brushed motor (DYN 1171) by Dynamite, and it is widely used in all popular Radio-Controlled trucks and cars. The technical information about the motor was minimal see Table 5.1. And therefore, most of the needed data were extracted physically from the motor parts after disassembled. This type of motor was chosen because of the reduced number of mechanical parts, price, easy availability, and compatibility with the available dynamometer.

Table 5.1: Dynamite 20-turn brushed motor specifications, Source: [150]

\begin{tabular}{|l|c|c|}
\hline 1 & Type & Brushed Size: 540 \\
\hline 2 & Bearings or Bushings & Bushing \\
\hline 3 & \# of Turns/Windings & 20T \\
\hline 4 & Timing & No Voltage: $4.8-9.6 \mathrm{~V}$ \\
\hline 5 & Cells & $4-8$ Ni-Cd/Ni-MH or $2 \mathrm{~S} \mathrm{Li-Po}$ \\
\hline 6 & Overall Diameter & 1.42 in. $(36 \mathrm{~mm})$ \\
\hline 7 & Shaft Diameter & $125 "(3.175 \mathrm{~mm})$ \\
\hline 8 & Shaft Length & 0.60 in. $(15.5 \mathrm{~mm})$ \\
\hline 9 & Overall Length & 1.98 in. $(50 \mathrm{~mm})$ \\
\hline
\end{tabular}

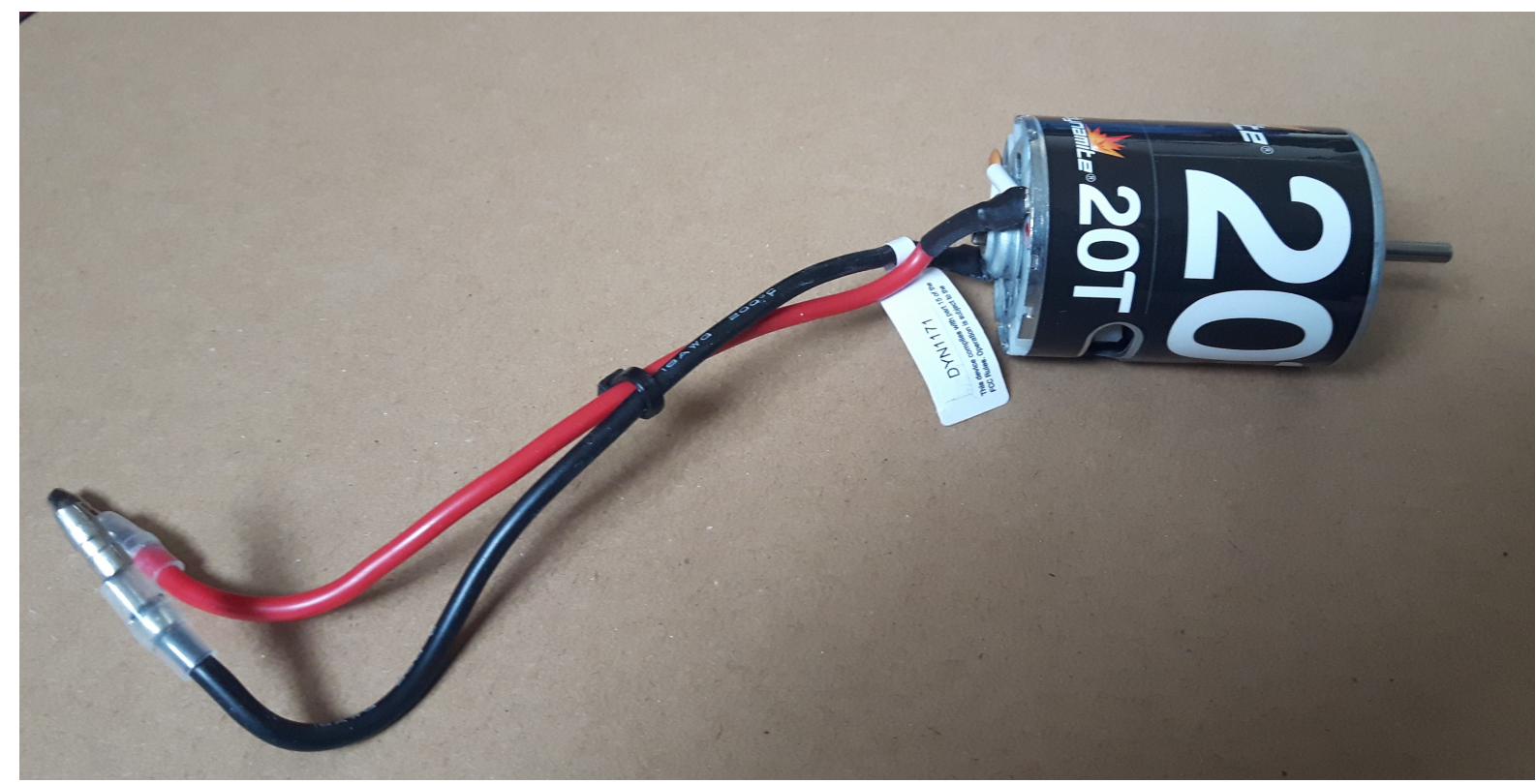

Figure 5.1: DC Permanent Magnet Motor 


\subsection{Motor disassembly}

When the motor was taken apart, its main components were:

- Stator: which includes motor end cab, bushings, carbon brushes, terminals.

- Rotor: (the dynamic part of the motor) includes motor commutator, motor shaft, armature core, and armature winding.

Figure5.2 shows the layout in an exploded view of a disassembled motor. The Armature is fixed on the shaft and has windings terminated to a commutator; motor terminals are attached to the motor windings through the motor brushes. The stator has two Neodymium magnets (NdFeB) $33.5 \mathrm{~mm}$ OD x $27.25 \mathrm{~mm}$ ID x $5 \mathrm{~mm}$ thick,140 degrees arc segments. The electromechanical motor is designed, so that opposite magnetic fields of the energized windings and the stator magnets make the shaft rotate. When the armature is aligned with the stator magnets, the brushes, which are also attached to the motor end-cab, will connect to the next commutator segment and energize the next winding. This will change the magnetic field of the armature, which makes the motor continue rotating [151]. The pictures below represent the stator, armature and commutator geometry in more detail.

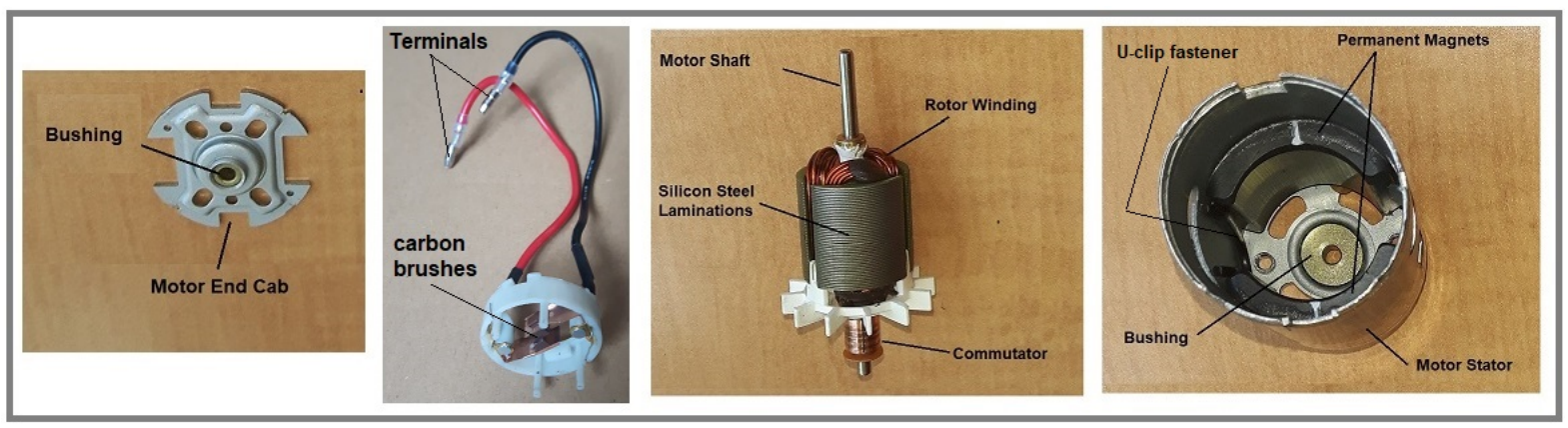

Figure 5.2: Motor Exploded View

\subsection{Rotor Winding}

The armature winding is the essential part of the rotating machine. It is the place where the electrical energy is converted into mechanical energy. The chosen motor's armature winding belongs to the lap winding classification as illustrated in Figure 5.3. In lap winding, the conductors are joined so that their parallel paths and poles are equal in number. The end of each armature coil is attached to the nearby segment on the commutator. The number of motor brushes in the lap winding is equivalent to the number of parallel paths, and these brushes are equally distributed into adverse and positive polarities [152].

The basic scheme of the chosen motor is straightforward. There is one coil; in each slot, the wire's current needs to flow in the same direction. The illustration's continuous lines are wires in the foreground, and dotted lines are 
wires in the background. While the basic scheme is simple, the actual winding is a bit more complex. The main problem for winding an electrical motor isn't the small space for wires in the slots, but all the overlapping and crossing wires going from one slot to another.

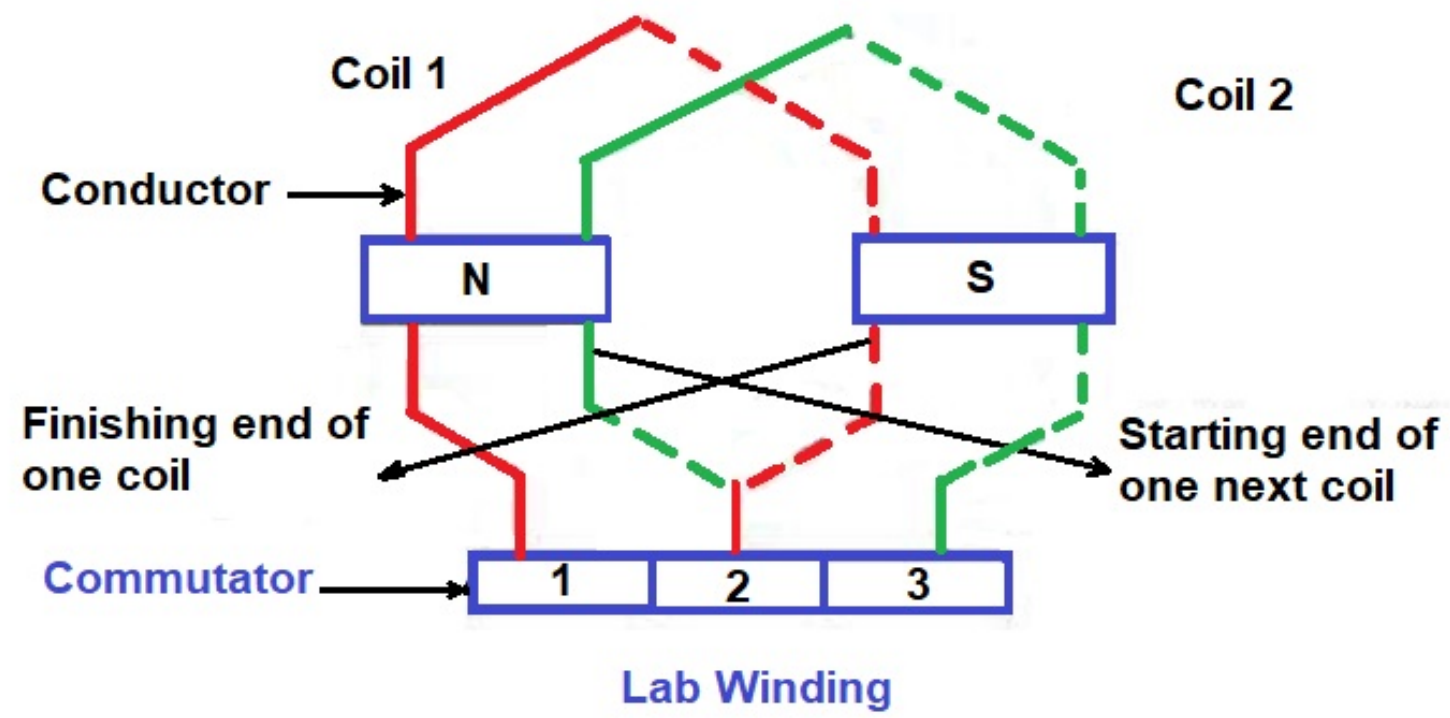

Figure 5.3: Lap Winding, Source: Adapted from [153].

\subsection{Motor Dimensions}

Access to a part's design documentation from its original production was not provided. Therefore, reverse engineering empowers us to analyze the physical motor parts and explore how it was originally made to replicate, create variations, or improve the design. The goal is to create a new CAD model for use in manufacturing ultimately. To extract accurate data, a digital electronic calliper was used to measure outside dimensions, inside dimensions, and depths of holes and slots, and the extracted data resulted in 2D and 3D CAD drawings, as illustrated in Appendix C.

\subsection{Motor Redesign}

For our redesign on the motor stator, we decided that we wanted to add two tongues to fix the permanent magnets instead of using the U-clip fastener that used in the traditional motor to provide strong clamping action for a firm, vibration-free for the PMs seen in Figure 5.2. To take advantage of using 3D printing techniques, the U-clip 
fastener was replaced by the tongues, which will simplify the process of assembling the PM's and make sure that we can accommodate the permanent magnets in the exact position without them being able to get close to each other as shown in Figure 5.4.

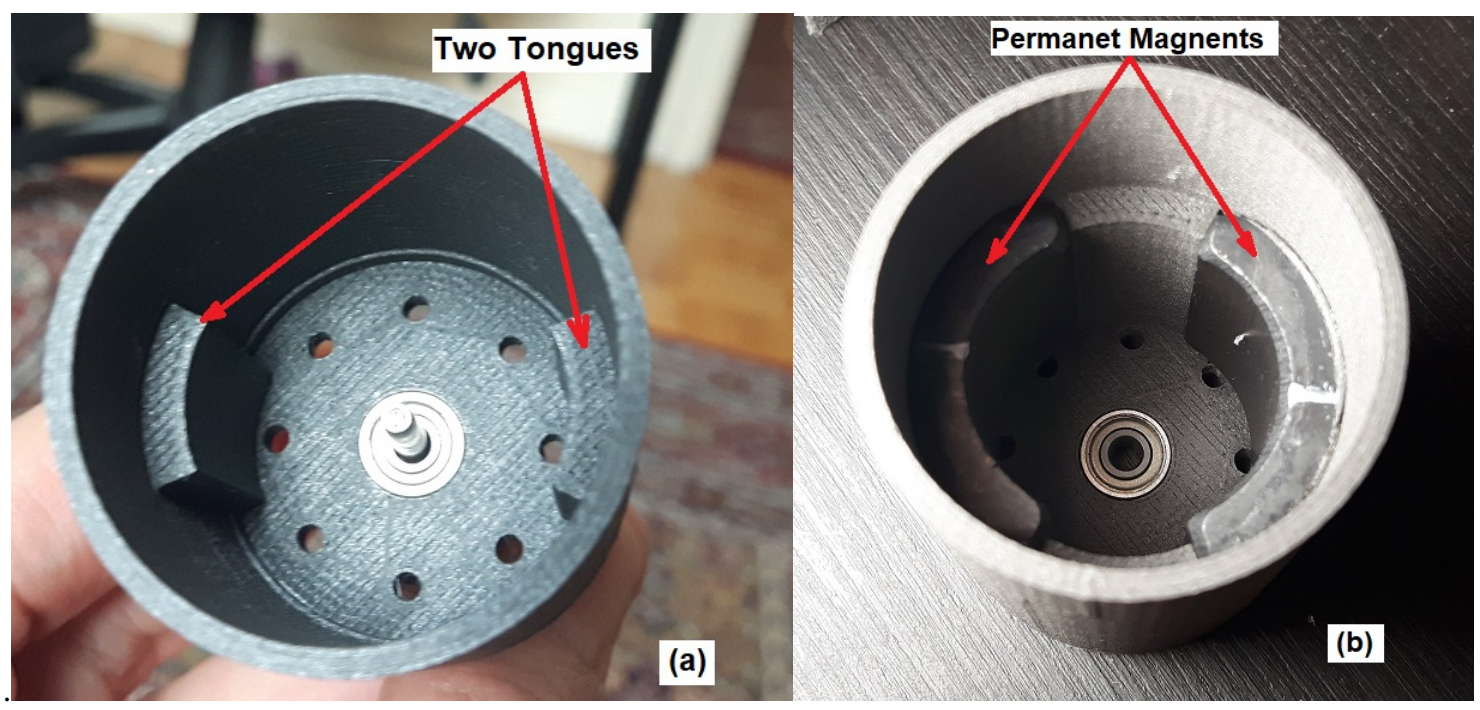

Figure 5.4: 3D Printed Motor Stator (a) Witout PM's (b) With PM's

\subsection{Fabrication (printing, assembly)}

The 3D-printed rotor, stator, end-cab and commutator shown in Figure 5.5 was built by the Anet 8A machine using magnetic iron PLA filament (see printing parametrs of magnetic iron PLA filament in appendix B). The motor parts are not very difficult to print; however, they need to be as precise as possible. We used high-resolution settings $(0.15 \mathrm{~mm}), 100 \%$ infill density, printed at slow speeds. Centrifugal forces are high, and an unbalanced rotor can cause big vibrations, noise, or maybe the motor can be damaged. Therefore, the motor shaft's hole in the 3d-model is exactly $3.175 \mathrm{~mm}$, and when printing, it will usually be a little smaller due to printing inaccuracies. That's desired because the shaft needs an accurate tight fit in the rotor. A drill press is used to adjust the bore exactly to the shaft using a low speed to avoid melting the 3d-printed material. Three equally spaced slots were created at the rotor core's outer circumference and used for winding the copper coil to form the poles. The poles were wound with copper coil $32 \mathrm{MAG}(0.85 \mathrm{~mm}$ diameter), and the number of turns per pole was 20 , as shown in Figure 5.6. The commutator was also 3D-printed using the same filament, and three sets of contact copper sheets $(0.254 \mathrm{~mm})$ were attached to the commutator. The rotor core and the commutator were attached to a steel axle of $3.175 \mathrm{~mm}$ diameter and $70 \mathrm{~mm}$ long, and the ends of the winding coils were soldered to the commutator copper tapes by hand. The electric power source was connected to the rotor winding through the commutator and 
brushes. Two stationary brushes contacted the commutator's copper conductor tapes to reverse the rotor's flow. Due to the small space available for the brushes, the same conventional motor brushes and terminals were used. The diameter of the core was $23 \mathrm{~mm}$, with a height of $22 \mathrm{~mm}$. The same number of turns, copper coil gage, and core dimensions will allow for superior comparisons between the 3D printed motor and the traditional motor. After rotor parts were assembled, and the magnets were placed firmly in their slots that created on the stator, the rotor shaft then pushed into the ball-bearing that placed firmly on the stator and the other side was pushed on the end-cab ball-bearing to form the completed assembly of the printed motor.

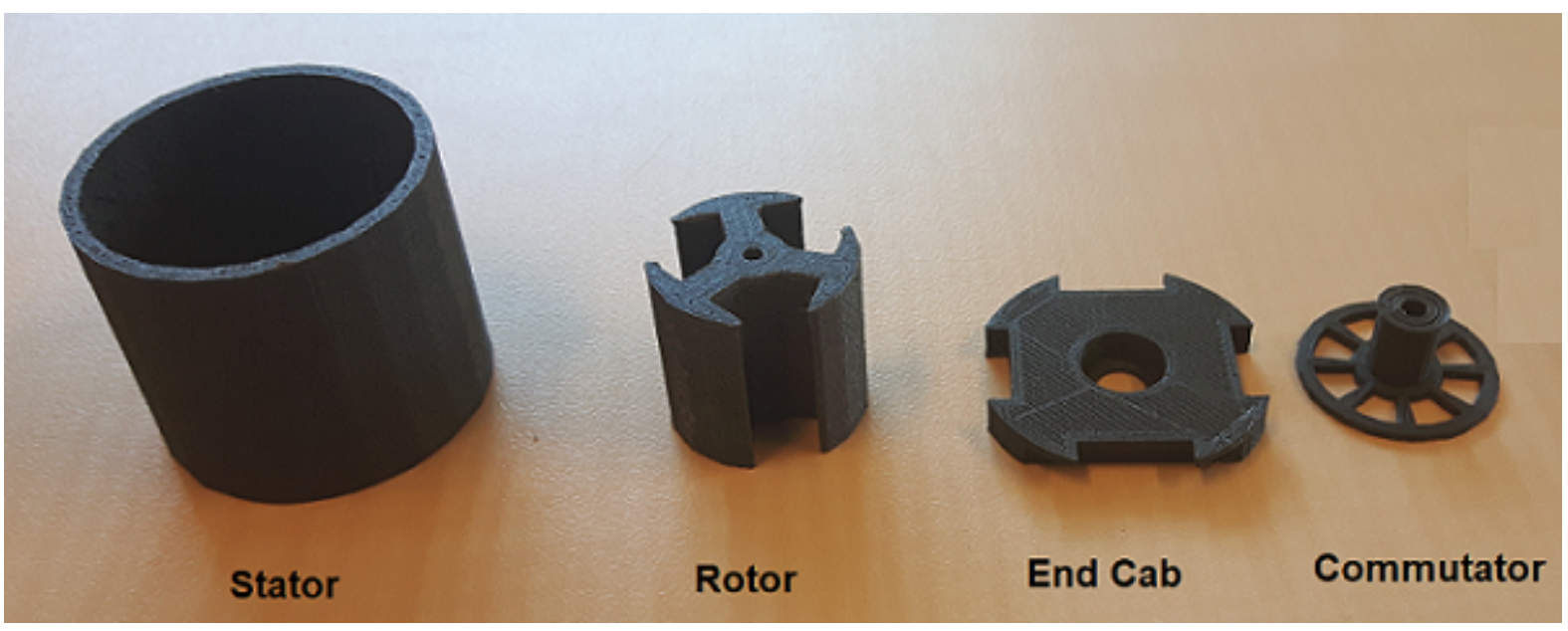

Figure 5.5: 3D printed Motor Parts 


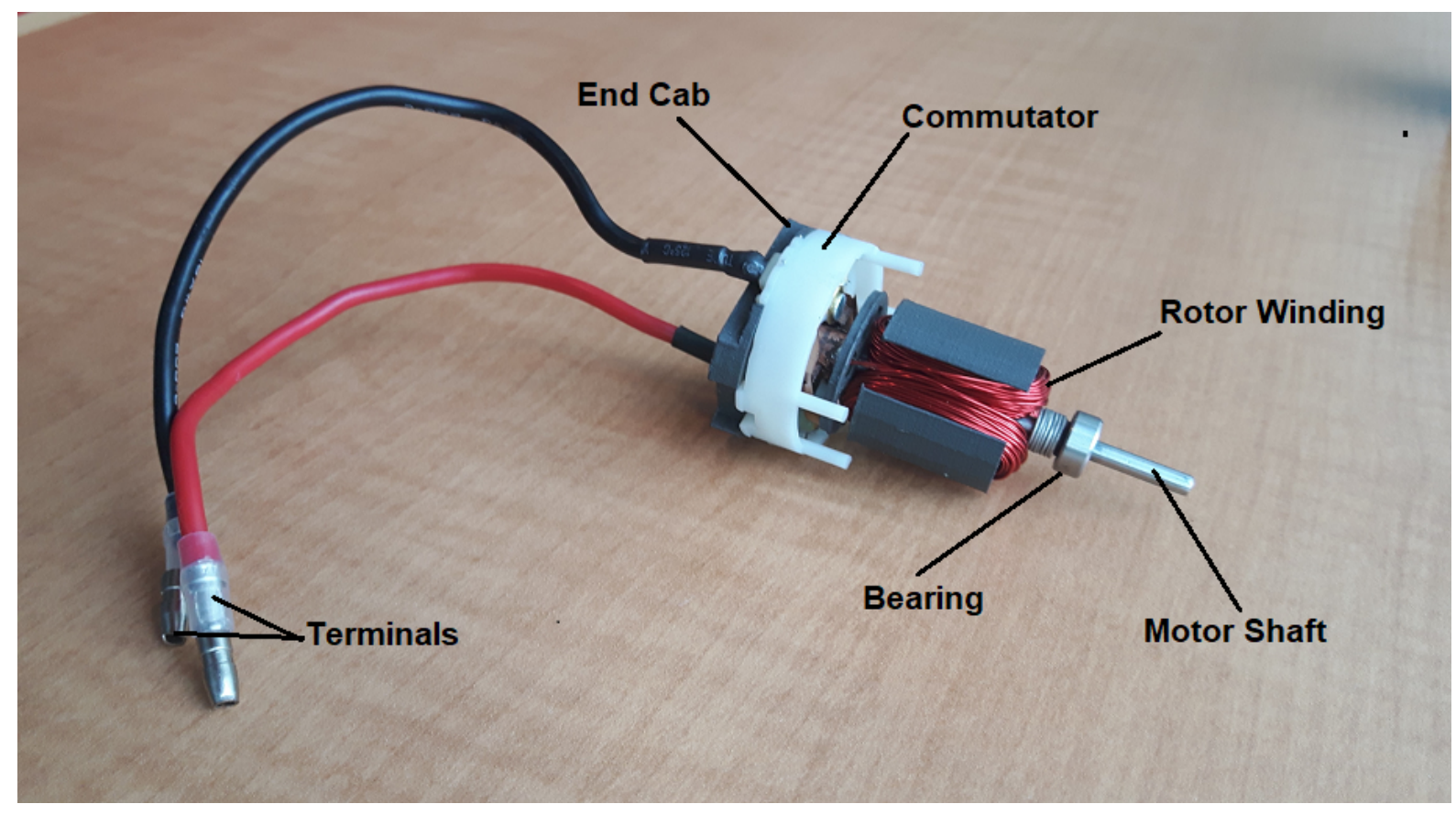

Figure 5.6: 3D Printed Rotor Assembly

\subsection{Results and Discussion}

Comparing the 3D printed motor to a commercially available off-the-shelf brushed DC motor allows for comparing how additive manufacturing can compete with traditional manufacturing. The evaluation consisted of comparing RPM vs. time for an identical start-up sequence of power. To determine these values, a universal benchtop inertia motor dynamometer (dyno) was used to measure the data. The complete setup of the testing devices consisted of a dynamometer, voltage and current sensor (miniPRO A1), electronic speed control (ESC), ESC controller sensor, battery, and laptop computer, as shown in Figure 5.7. 


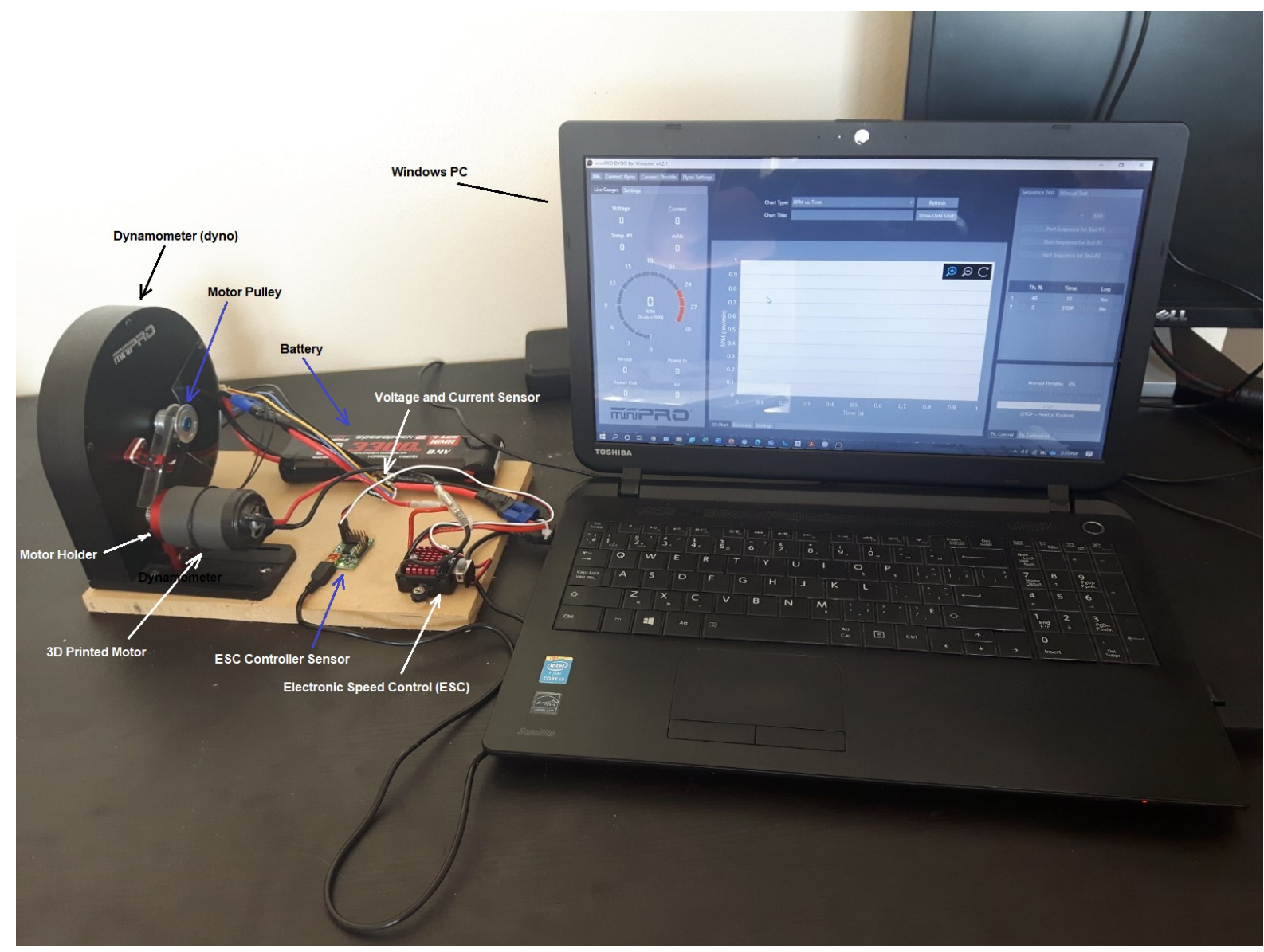

Figure 5.7: The Complete Setup of the Testing Devices

The motors were mounted to the motor holder consecutively. The dyno is connected to a motor holder via a pulley and belt to accelerate the inertial mass using a flywheel. The dyno uses the motor to accelerate a flywheel and calculates the motor's mechanical power and mechanical torque by knowing the flywheel's inertia and its acceleration. The test of each electric motor was performed time after time until the same results were obtained. Every test was repeated under the same conditions using the electronic speed controller (type 60A Brushed Waterproof by Dynamite). The electronic speed controller is used to control the motor's speed.

Each motor attached to the dyno motor holder was connected to an $8.4 \mathrm{~V} \mathrm{3300mAh} \mathrm{battery} \mathrm{(type} \mathrm{7-Cell} \mathrm{NiMH}$ Flat EC3 by Dynamite) through the speed controller and the voltage and current sensor. The speed controller is also connected to the ESC controller sensor then to the laptop computer. The dyno and the Throttle Controller Sensor are connected to the laptop using two micro-b USB cables. Tests then were performed by the dyno software, and the evaluation was consisted of comparing RPM vs. time.

Firstly, the commercial motor was mounted on the test bench to extract the needed data and use it as a reference for the comparison method. Secondly, the 3D printed rotor was assembled with the commercial motor's stator 
(partially 3D printed motor) to test the 3D printed motor rotor's behaviour before assembling the complete printed motor. Finally, the 3D printed stator and rotor were assembled together (fully 3D printed motor) and mounted on the test bench to evoke the required data and compare it with previously obtained data. In order to compare the three tests, the obtained results were combined together in one graph. The three motors were accelerated to $100 \%$ throttle for 10 seconds, and results showed that they performed differently under the same condition as shown in Figur 5.8.

\section{TEST RESULTS GRAPH}

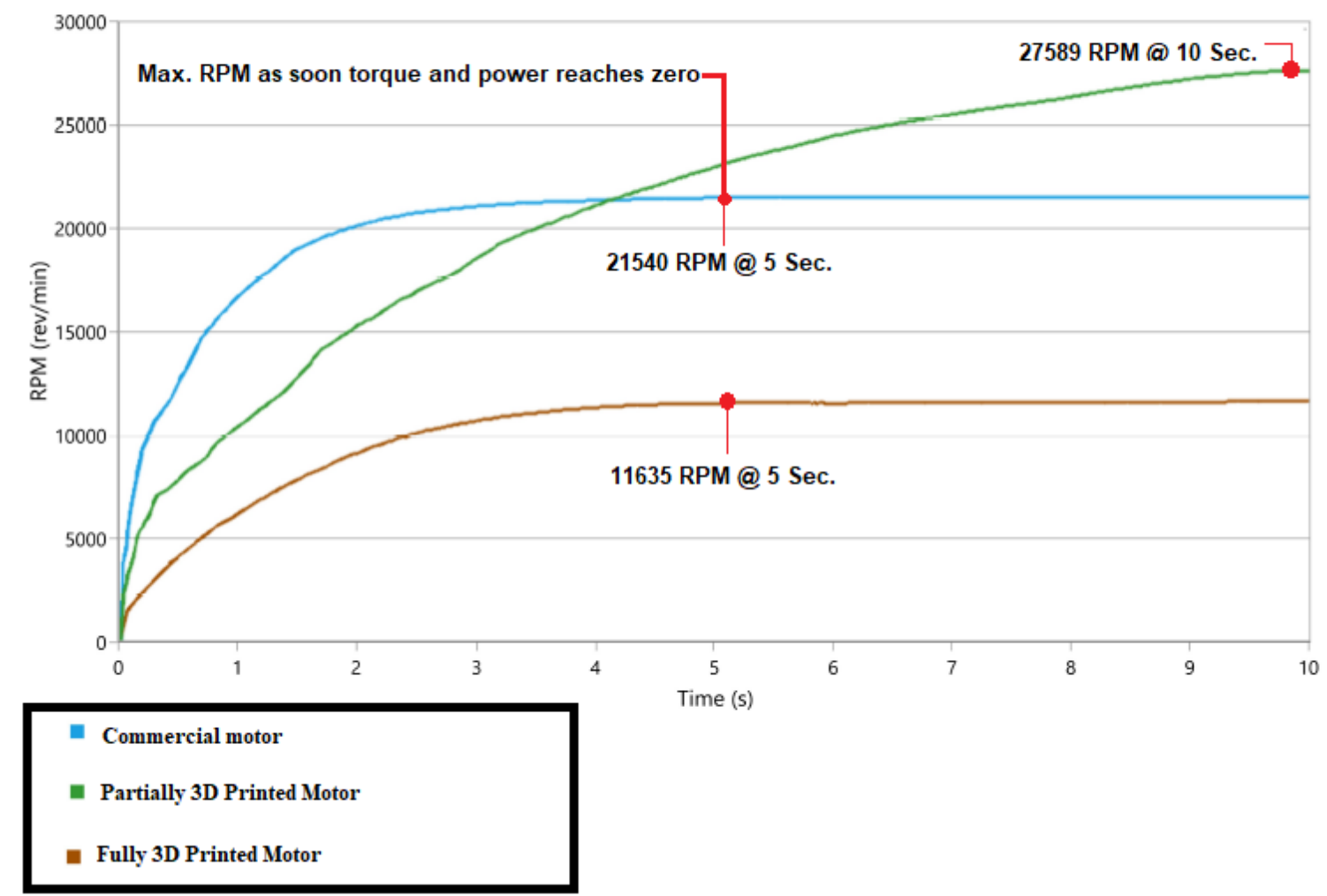

Figure 5.8: RPM Comparison

\subsection{Conclusions.}

The graph shows the RPM versus the time of the three tests. The commercial motor accelerates from ( 0 to 21540 RPM) in about 5 seconds; this point represents the maximum RPM as soon torque and power reach zero. In comparison, the partially 3D printed motor that is constructed from a 3D printed rotor and the commercial stator reaches its maximum speed (27589 RPM) in about 10 seconds. The fully 3D printed motor performance was less 
than the other two motors in terms of its maximum speed; it reaches its maximum speed (11635 RPM) in about 5 seconds, and therefore, further investigation has to be performed in order to increase its performance. 


\section{Chapter 6}

\section{Conclusions and Future Work}

\subsection{Conclusion}

We have demonstrated a DC electric motor in which only the wire coils are not 3D-printed. However, the coils can be replaced by copper foil, which can be 3D-printed using the LOM technique. The Circuit Explore Air 2 machine was used to form the shape of the pancake motor armature winding instead of the LOM machine; further investigation is recommended to confirm this technique. All parts of the radial and axial flux DC electric motors were fabricated with 3D printing techniques. The functionality indicated the potential to achieve a fully 3D-printed motor that may replace the traditional motor using inexpensive, commercially available equipment. Based on the analysis of the built prototypes, The main conclusions can be summarized as follows:

- In the radial-flux motor design, The magnetic iron PLA rotor has the highest start-up torque at the highest speeds than the other two silicon steel rotors due to its advantages of no eddy current losses.

- The closed stator geometry in the radial-flux motor design provides a low reluctance path for the magnetic flux compared to the open geometry. And therefore, it produced more intense EMF than the open geometry.

- In the axial-flux motor design, the motor rotors made using a LOM-like process of the axial-flux motor indicated the potential of a 3D-printed pattern to replace the copper wire using the LOM technique.

- The fully 3D printed motor has lower torque and lower speed when benchmarked with the traditional motor, which inquires further investigation to increase performance. 
Though manual intervention was used in these samples to build the motors, the systems demonstrate what we can do with a multi-material, multi-technology 3D printer. A promising idea for such a machine's future application is to facilitate evolving a self-replicating machine, which is considered a tool necessary for outer space exploration. If fully achieved, it will demonstrate that 3D printing could lead to sustainability in space exploration, remote regions on earth, etc.

\subsection{Future Work}

To better understand these results' implications, further research is required to analyze the $3 \mathrm{D}$ printed motor theoretically using the proper software tool until the desired performance is satisfied. Identifying an optimal design can be determined once we know the mechanical, thermal, and magnetic properties of the materials we are working on within additive manufacturing. Benchmarking material properties from each AM process in various orientations against the typical material performance for rotors, stators, commutators, and PMs produced using conventional methods could also inform the decision on material and process selection. The material properties could also be used in simulations for design optimization to leverage the true potentials of AM towards the production of novel motor products. Further research is also needed to determine the possibility of using a LOM technique to build the motor on two different machines. Future studies could also address finding a motor design that maximizes power efficiency. This may require many processes includes integrating several manufacturing technologies into a single system, multiple metal and polymer extrusion technologies, machining, copper wire extrusion and soldering. If possible, the motors described here will be fabricated by a single machine without human involvement. Ultimately, if 3D-printed electric motors can be achieved in a single system, it will show that they can substantially improve space exploration technological development and reduce costs. 


\section{Appendix A}

This appendix provides details about the 3D printed pole shoes which ordered and received from ICAMP Innovation Centre that operated by Canadore College in North Bay, Ontario. 


\section{eiss}

Material data sheet

\section{EOS MaragingSteel MS1}

EOS MaragingSteel MS1 is a steel powder which has been optimized especially for processing on EOSINT M systems.

This document provides information and data for parts built using EOS MaragingSteel MS1 powder (EOS art.-no. 9011-0016) on the following system specifications:

- EOSINT M 270 Installation Mode Standard with PSW 3.3 or 3.4 and default job MS1_020_default.job or MS1_040_default.job

- EOSINT M 270 Dual Mode with PSW 3.5 and EOS Original Parameter Set MS1_Surface 1.0 or MS1_Performance 2.0

- EOSINT M 280 with PSW 3.5 and EOS Original Parameter Set MS1_Performance 1.0 or MS1_Speed 1.0

\section{Description}

Parts built in EOS MaragingSteel MS1 have a chemical composition corresponding to US classification 18\% Ni Maraging 300, European 1.2709 and German X3NiCoMoTi 18-9-5. This kind of steel is characterized by having very good mechanical properties, and being easily heattreatable using a simple thermal age-hardening process to obtain excellent hardness and strength.

Parts built from EOS MaragingSteel MS1 are easily machinable after the building process and can be easily post-hardened to more then $50 \mathrm{HRC}$ by age-hardening at $490^{\circ} \mathrm{C}\left(914^{\circ} \mathrm{F}\right)$ for 6 hours. In both as-built and age-hardened states the parts can be machined, spark-eroded, welded, micro shot-peened, polished and coated if required. Due to the layerwise building method, the parts have a certain anisotropy, which can be reduced or removed by appropriate heat treatment - see Technical Data for examples. 


\section{Material data sheet}

\section{Technical data}

\section{General process data}

\begin{tabular}{|c|c|}
\hline \multicolumn{2}{|l|}{ Typical achievable part accuracy [1] } \\
\hline - small parts $(<80 \times 80 \mathrm{~mm})$ & $\begin{array}{l}\text { approx. } \pm 20 \mu \mathrm{m} \\
\text { approx. } \pm 0.8 \times 10^{-3} \mathrm{inch}\end{array}$ \\
\hline - large parts & $\begin{array}{l}\text { approx. } \pm 50 \mu \mathrm{m} \\
\text { approx. } \pm 0.002 \text { inch }\end{array}$ \\
\hline Age hardening shrinkage [2] & approx. $0.08 \%$ \\
\hline Min. wall thickness [3] & $\begin{array}{l}\text { approx. } 0.3-0.4 \mathrm{~mm} \\
\text { approx. } 0.012-0.016 \text { inch }\end{array}$ \\
\hline \multicolumn{2}{|l|}{ Surface roughness (approx.) [4] } \\
\hline - as manufactured & \\
\hline MS1 Surface $(20 \mu \mathrm{m})$ & $\begin{array}{l}\text { Ra } 4 \mu \mathrm{m} ; \mathrm{Rz} 20 \mu \mathrm{m} \\
\text { Ra } 0.16 \times 10^{-3} \mathrm{inch}, \\
R_{z} 0.78 \times 10^{-3} \mathrm{inch}\end{array}$ \\
\hline MS1 Performance (40 m) & $\begin{array}{l}\text { Ra } 5 \mu \mathrm{m} ; \mathrm{Rz} 28 \mu \mathrm{m} \\
\text { Ra } 0.19 \times 10^{-3} \mathrm{inch}, \\
\text { Rz } 1.10 \times 10^{-3} \mathrm{inch}\end{array}$ \\
\hline MS1 Speed $(50 \mu \mathrm{m})$ & $\begin{array}{l}\text { Ra } 9 \mu \mathrm{m} ; \mathrm{Rz} 50 \mu \mathrm{m} \\
\text { Ra } 0.47 \times 10^{-3} \text { inch, } \\
R_{z} 2.36 \times 10^{-3} \text { inch }\end{array}$ \\
\hline - after shot-peening & $\begin{array}{l}R_{a} 4-6.5 \mu \mathrm{m} ; R_{z} 20-50 \mu \mathrm{m} \\
R_{a} 0.16-0.26 \times 10^{-3} \mathrm{inch} \\
R_{z} 0.78-1.97 \times 10^{-3} \mathrm{inch}\end{array}$ \\
\hline - after polishing & $\begin{array}{l}R_{z} \text { up to }<0.5 \mu \mathrm{m} \\
\mathrm{Rz}_{z} \text { up to }<0.02 \times 10^{-3} \mathrm{inch} \\
\text { (can be very finely polished) }\end{array}$ \\
\hline
\end{tabular}




\section{Material data sheet}

\begin{tabular}{|c|c|}
\hline Volume rate [5] & \\
\hline $\begin{array}{l}\text { - Parameter set MS1_Surface } 1.0 \text { / default job } \\
\text { MS1_020_default.job (20 } \mu \mathrm{m} \text { layer thickness) }\end{array}$ & $\begin{array}{c}1.6 \mathrm{~mm}^{3} / \mathrm{s}\left(5.8 \mathrm{~cm}^{3} / \mathrm{h}\right) \\
0.35 \mathrm{in}^{3} / \mathrm{h}\end{array}$ \\
\hline $\begin{array}{l}\text { - Parameter set MS1_Performance } 2.0 \text { / default job } \\
\text { MS1_040_default.job (40 } \mu \mathrm{m} \text { layer thickness) }\end{array}$ & $\begin{array}{c}3 \mathrm{~mm}^{3} / \mathrm{s}\left(10.8 \mathrm{~cm}^{3} / \mathrm{h}\right) \\
0.66 \mathrm{in}^{3} / \mathrm{h}\end{array}$ \\
\hline $\begin{array}{l}\text { - Parameter set MS1_Performance } 1.0 \text { / for M } 280 / 400 \text { W } \\
\text { (40 } \mu \mathrm{m} \text { layer thickness) }\end{array}$ & $\begin{array}{c}4.2 \mathrm{~mm}^{3} / \mathrm{s}\left(15.1 \mathrm{~cm}^{3} / \mathrm{h}\right) \\
0.92 \mathrm{in}^{3} / \mathrm{h}\end{array}$ \\
\hline $\begin{array}{l}\text { - Parameter set MS1_Speed } 1.0 \text { / for M } 280 \text { / } 400 \text { W } \\
\text { (50 } \mu \mathrm{m} \text { layer thickness) }\end{array}$ & $\begin{array}{c}5.5 \mathrm{~mm}^{3} / \mathrm{s}\left(19.8 \mathrm{~cm}^{3} / \mathrm{h}\right) \\
1.21 \mathrm{in}^{3} / \mathrm{h}\end{array}$ \\
\hline
\end{tabular}

[1] Based on users' experience of dimensional accuracy for typical geometries, as built. Part accuracy is subject to appropriate data preparation and post-processing, in accordance with EOS training.

[2] Ageing temperature $490^{\circ} \mathrm{C}\left(914^{\circ} \mathrm{F}\right), 6$ hours, air cooling

[3] Mechanical stability is dependent on geometry (wall height etc.) and application

[4] Due to the layerwise building, the surface structure depends strongly on the orientation of the surface, for example sloping and curved surfaces exhibit a stair-step effect. The values also depend on the measurement method used. The values quoted here given an indication of what can be expected for horizontal (up-facing) or vertical surfaces.

[5] Volume rate is a measure of build speed during laser exposure of hatched areas. The total build speed depends on the average volume rate, the recoating time (related to the number of layers) and other geometry- and machine setting-related factors. 


\section{Material data sheet}

Physical and chemical properties of parts

\begin{tabular}{lc}
\hline Material composition & Fe (balance) \\
& Ni $(17-19 \mathrm{wt}-\%)$ \\
Co $(8.5-9.5 \mathrm{wt}-\%)$ & $\mathrm{Mo}(4.5-5.2 \mathrm{wt}-\%)$ \\
$\mathrm{Ti}(0.6-0.8 \mathrm{wt}-\%)$ \\
$\mathrm{Al}(0.05-0.15 \mathrm{wt}-\%)$ \\
$\mathrm{Cr}, \mathrm{Cu}($ each $\leq 0.5 \mathrm{wt}-\%)$ \\
$\mathrm{C}(\leq 0.03 \mathrm{wt}-\%)$ \\
$\mathrm{Mn}, \mathrm{Si}($ each $\leq 0.1 \mathrm{wt}-\%)$ \\
$\mathrm{P}, \mathrm{S}($ each $\leq 0.01 \mathrm{wt}-\%)$ \\
\hline Relative density & approx. $100 \%$ \\
\hline Density & $8.0-8.1 \mathrm{~g} / \mathrm{cm}^{3}$ \\
& $0.289-0.293 \mathrm{lb} / \mathrm{in}^{3}$ \\
\hline
\end{tabular}




\section{Material data sheet}

\section{Mechanical properties of parts at $20^{\circ} \mathrm{C}\left(68^{\circ} \mathrm{F}\right)$}

\begin{tabular}{|c|c|c|}
\hline & As built & After age hardening [2] \\
\hline Tensile strength [6] & & $\begin{array}{l}\min .1930 \mathrm{MPa} \\
\min .280 \mathrm{ksi}\end{array}$ \\
\hline - in horizontal direction (XY) & $\begin{array}{l}\text { typ. } 1100 \pm 100 \mathrm{MPa} \\
\text { typ. } 160 \pm 15 \mathrm{ksi}\end{array}$ & \multirow{2}{*}{$\begin{array}{l}\text { typ. } 2050 \pm 100 \mathrm{MPa} \\
\text { typ. } 297 \pm 15 \mathrm{ksi}\end{array}$} \\
\hline - in vertical direction (Z) & $\begin{array}{c}\text { typ. } 1100 \pm 100 \mathrm{MPa} \\
\text { typ. } 160 \pm 15 \mathrm{ksi}\end{array}$ & \\
\hline Yield strength (Rp $0.2 \%)$ [6] & & $\begin{array}{c}\min .1862 \mathrm{MPa} \\
\min .270 \mathrm{ksi}\end{array}$ \\
\hline - in horizontal direction (XY) & $\begin{array}{l}\text { typ. } 1050 \pm 100 \mathrm{MPa} \\
\text { typ. } 152 \pm 15 \mathrm{ksi}\end{array}$ & \multirow{2}{*}{$\begin{array}{l}\text { typ. } 1990 \pm 100 \mathrm{MPa} \\
\text { typ. } 289 \pm 15 \mathrm{ksi}\end{array}$} \\
\hline - in vertical direction (Z) & $\begin{array}{l}\text { typ. } 1000 \pm 100 \mathrm{MPa} \\
\text { typ. } 145 \pm 15 \mathrm{ksi}\end{array}$ & \\
\hline Elongation at break [6] & & $\min .2 \%$ \\
\hline - in horizontal direction (XY) & typ. $(10 \pm 4) \%$ & \multirow{2}{*}{ typ. $(4 \pm 2) \%$} \\
\hline - in vertical direction (Z) & typ. $(10 \pm 4) \%$ & \\
\hline \multicolumn{3}{|l|}{ Modulus of elasticity [6] } \\
\hline - in horizontal direction (XY) & $\begin{array}{l}\text { typ. } 160 \pm 25 \mathrm{GPa} \\
\text { typ. } 23 \pm 4 \mathrm{Msi}\end{array}$ & \multirow{2}{*}{$\begin{array}{l}\text { typ. } 180 \pm 20 \mathrm{GPa} \\
\text { typ. } 26 \pm 3 \mathrm{Msi}\end{array}$} \\
\hline - in vertical direction (Z) & $\begin{array}{l}\text { typ. } 150 \pm 20 \mathrm{GPa} \\
\text { typ. } 22 \pm 3 \mathrm{Msi}\end{array}$ & \\
\hline Hardness [7] & typ. $33-37$ HRC & typ. $50-56$ HRC \\
\hline Ductility (Notched Charpy impact test) & typ. $45 \pm 10 \mathrm{~J}$ & typ. $11 \pm 4 \mathrm{~J}$ \\
\hline
\end{tabular}

[6] Tensile testing according to ISO 6892-1:2009 (B) Annex D, proportional test pieces, diameter of the neck area $5 \mathrm{~mm}$ ( 0.2 inch), original gauge length $25 \mathrm{~mm}$ ( 1 inch).

[7] Rockwell C (HRC) hardness measurement according to EN ISO 6508-1 on polished surface. Note that measured hardness can vary significantly depending on how the specimen has been prepared. 


\title{
Material data sheet
}

\section{Thermal properties of parts}

\begin{tabular}{lcc}
\hline & As built & After age hardening [2] \\
\hline Thermal conductivity & $\begin{array}{c}\text { typ. } 15 \pm 0.8 \mathrm{~W} / \mathrm{m}^{\circ} \mathrm{C} \\
\text { typ. } 104 \pm 6 \mathrm{Btu} \mathrm{in} /\left(\mathrm{h} \mathrm{ft}{ }^{\circ} \mathrm{F}\right)\end{array}$ & $\begin{array}{r}\text { typ. } 20 \pm 1 \mathrm{~W} / \mathrm{m}^{\circ} \mathrm{C} \\
\text { typ. } 139 \pm 7 \mathrm{Btu} \mathrm{in} /\left(\mathrm{h} \mathrm{ft}{ }^{\circ} \mathrm{F}\right)\end{array}$ \\
\hline Specific heat capacity & typ. $450 \pm 20 \mathrm{~J} / \mathrm{kg}^{\circ} \mathrm{C}$ & typ. $450 \pm 20 \mathrm{~J} / \mathrm{kg}^{\circ} \mathrm{C}$ \\
& typ. $0.108 \pm 0.005 \mathrm{Btu} /\left(\mathrm{lb}{ }^{\circ} \mathrm{F}\right)$ & typ. $0.108 \pm 0.005 \mathrm{Btu} /\left(\mathrm{l} \mathrm{\circ}^{\circ} \mathrm{F}\right)$ \\
\hline Maximum operating temperature & & approx. $400^{\circ} \mathrm{C}$ \\
& & approx. $750^{\circ} \mathrm{F}$ \\
\hline
\end{tabular}

\section{Abbreviations}

\author{
typ. typical \\ min. minimum \\ approx. approximately \\ wt weight
}

\section{Notes}

The data are valid for the combinations of powder material, machine and parameter sets referred to on page 1 , when used in accordance with the relevant Operating Instructions (including Installation Requirements and Maintenance) and Parameter Sheet. Part properties are measured using defined test procedures. Further details of the test procedures used by EOS are available on request. Unless otherwise specified, the data refer to the default job MS1_040_default.job or the equivalent parameter set MS1_Performance 2.0. The corresponding data for the default job MS1_020_default.job or the equivalent parameter set MS1_Surface 1.0 are approximately the same except where otherwise specified.

The data correspond to our knowledge and experience at the time of publication. They do not on their own provide a sufficient basis for designing parts. Neither do they provide any agreement or guarantee about the specific properties of a part or the suitability of a part for a specific application. The producer or the purchaser of a part is responsible for checking the properties and the suitability of a part for a particular application. This also applies regarding any rights of protection as well as laws and regulations. The data are subject to change without notice as part of EOS' continuous development and improvement processes.

EOS $^{\circledR}$, EOSINT $^{\circledR}$ and DMLS ${ }^{\circledR}$ are registered trademarks of EOS GmbH.

(c) 2011 EOS GmbH - Electro Optical Systems. All rights reserved. 


\section{Appendix B}

This appendix provides details of printing parameters.

Printing Parameters of Magnetic Iron PLA Filament

Print Setup

= Quality

Layer Height

Initial Layer Height

Infill Line Width

Did shell

Wall Thickness

Top/Bottom Thickness

X Infill

Infill Density

||||| Material

Printing Temperature

Build Plate Temperature

Diameter

Flow

Enable Retraction

Retraction Distance

Retraction Speed

(O) Speed

Print Speed

Top/Bottom Speed

Travel Speed

Support

Enable Support

* Build Plate Adhesion

Build Plate Adhesion Type

Skirt Line Count

\section{Recommended Custom}

\begin{tabular}{|lc|}
\multicolumn{1}{c}{} & \multicolumn{1}{c}{} \\
\hline 0.15 & $\mathrm{~mm}$ \\
\hline \hline 0.3 & $\mathrm{~mm}$ \\
\hline \hline 0.4 & $\mathrm{~mm}$ \\
\hline
\end{tabular}

$\checkmark$

\begin{tabular}{|lc|}
\hline 1 & $\mathrm{~mm}$ \\
\hline \hline 0.8 & $\mathrm{~mm}$ \\
\hline & $i \smile$
\end{tabular}

? 100

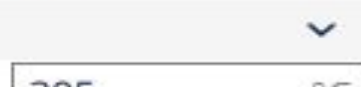

\begin{tabular}{|lr|}
\hline 205 & ${ }^{\circ} \mathrm{C}$ \\
\hline \hline 60 & ${ }^{\circ} \mathrm{C}$ \\
\hline \hline 1.75 & $\mathrm{~mm}$ \\
\hline \hline 100 & 96 \\
\hline
\end{tabular}

$\checkmark$

\begin{tabular}{|lr|}
\hline 6.5 & $\mathrm{~mm}$ \\
\hline \hline 25 & $\mathrm{~mm} / \mathrm{s}$ \\
\hline
\end{tabular}

$i \smile$

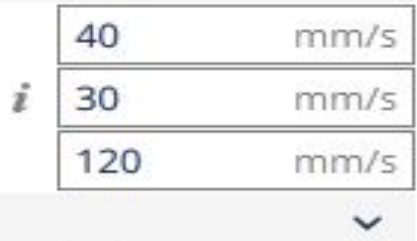

$\curvearrowright \square$

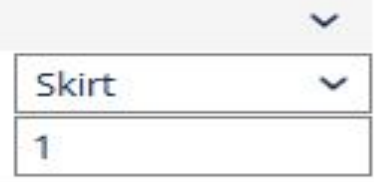


Printing Parameters of ABS Filament

\section{Print Setup}

Infill Line Width

I8 Shell

Wall Thickness

Top/Bottom Thickness

ख Infill

Infill Density

||||| Material

Printing Temperature

Build Plate Temperature

Diameter

Flow

Enable Retraction

Retraction Distance

Retraction Speed

(7) Speed

Print Speed

Top/Bottom Speed

Travel Speed

\section{Support}

Enable Support

Support Overhang Angle

Support Pattern

Support Density

\section{Recommended Custom}

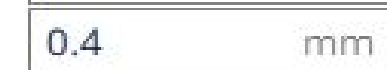

\begin{tabular}{|ll|}
\hline 1 & $\mathrm{~mm}$ \\
\hline \hline 0.8 & $\mathrm{~mm}$ \\
\hline
\end{tabular}

$\checkmark$

$20 \quad 96$

$205 \quad{ }^{\circ} \mathrm{C}$

$80 \quad{ }^{\circ} \mathrm{C}$

$1.75 \mathrm{~mm}$

100

$\checkmark$

$6.5 \mathrm{~mm}$

$25 \mathrm{~mm} / \mathrm{s}$
$i \vee$

$i$\begin{tabular}{lc|}
\hline 40 & $\mathrm{~mm} / \mathrm{s}$ \\
\hline \hline 30 & $\mathrm{~mm} / \mathrm{s}$ \\
\hline \hline 120 & $\mathrm{~mm} / \mathrm{s}$ \\
\hline
\end{tabular}

\begin{tabular}{|ll|}
\hline$\checkmark$ & \\
\hline 50 & $\circ$ \\
\hline \hline Zig Zag & $\checkmark$ \\
\hline 15 & $\% 6$ \\
\hline
\end{tabular}


Printing Parameters of Conductive Copper Filament (Electrifi by Multi3D)

Print Setup

= Quality

Layer Height

Initial Layer Height

Infill Line Width

\section{DS Shell}

Wall Thickness

Top/Bottom Thickness

Infill

Infill Density

||||| Material

Printing Temperature

Build Plate Temperature

Diameter

Flow

Enable Retraction

Retraction Distance

Retraction Speed

(7) Speed

Print Speed

Top/Bottom Speed

Travel Speed

Support

Enable Support

* Build Plate Adhesion

Build Plate Adhesion Type

\section{Recommended Custom}

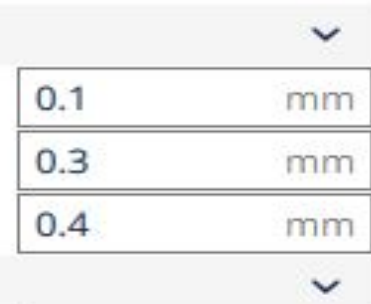

\begin{tabular}{|cc|}
\hline 0.8 & $\mathrm{~mm}$ \\
\hline \hline 0.8 & $\mathrm{~mm}$ \\
\hline & $\checkmark$
\end{tabular}

$20 \quad 96$

$200 \quad{ }^{\circ} \mathrm{C}$

$60 \quad{ }^{\circ} \mathrm{C}$

$2.85 \mathrm{~mm}$

$100 \quad 96$

$\checkmark$

$6.5 \mathrm{~mm}$

$25 \mathrm{~mm} / \mathrm{s}$

\begin{tabular}{|ll|}
\hline 60 & $\mathrm{~mm} / \mathrm{s}$ \\
\hline \hline 30.0 & $\mathrm{~mm} / \mathrm{s}$ \\
\hline 120 & $\mathrm{~mm} / \mathrm{s}$ \\
\hline
\end{tabular}
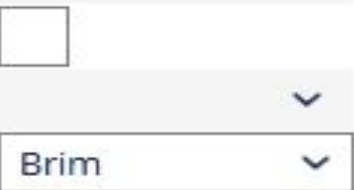
Printing Parameters of PLA Filament

Print Setup

= Quality

Layer Height

Initial Layer Height

Infill Line Width

II. shell

Wall Thickness

Top/Bottom Thickness

ख Infill

Infill Density

||||| Material

Printing Temperature

Build Plate Temperature

Diameter

Flow

Enable Retraction

Retraction Distance

Retraction Speed

(2) Speed

Print Speed

Top/Bottom Speed

Travel Speed

Support

Enable Support

$\div$ Build Plate Adhesion

Build Plate Adhesion Type

\section{Recommended Custom}

\begin{tabular}{|ll|}
\hline 0.25 & $\mathrm{~mm}$ \\
\hline \hline 0.25 & $\mathrm{~mm}$ \\
\hline \hline 0.4 & $\mathrm{~mm}$ \\
\hline
\end{tabular}

$\checkmark$

\begin{tabular}{|cc|}
\hline 0.4 & $\mathrm{~mm}$ \\
\hline \hline 0.8 & $\mathrm{~mm}$ \\
\hline & $\sim$
\end{tabular}

$100 \quad 96$

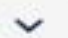

\begin{tabular}{|lr|}
\hline 160 & ${ }^{\circ} \mathrm{C}$ \\
\hline \hline 0 & ${ }^{\circ} \mathrm{C}$ \\
\hline \hline 1.75 & $\mathrm{~mm}$ \\
\hline \hline 100 & $\% 6$ \\
\hline
\end{tabular}

$\checkmark$

\begin{tabular}{lr}
\hline 1 & $\mathrm{~mm}$ \\
\hline \hline 25 & $\mathrm{~mm} / \mathrm{s}$
\end{tabular}

$i \vee$

$i$\begin{tabular}{|ll|}
\hline 15 & $\mathrm{~mm} / \mathrm{s}$ \\
\hline \hline 15 & $\mathrm{~mm} / \mathrm{s}$ \\
\hline \hline 120 & $\mathrm{~mm} / \mathrm{s}$ \\
\hline
\end{tabular}

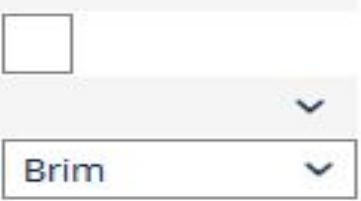




\section{Appendix C}

This appendix provides details of 2D, and 3D CAD drawings of the 3D printed motor parts in chapter 5.

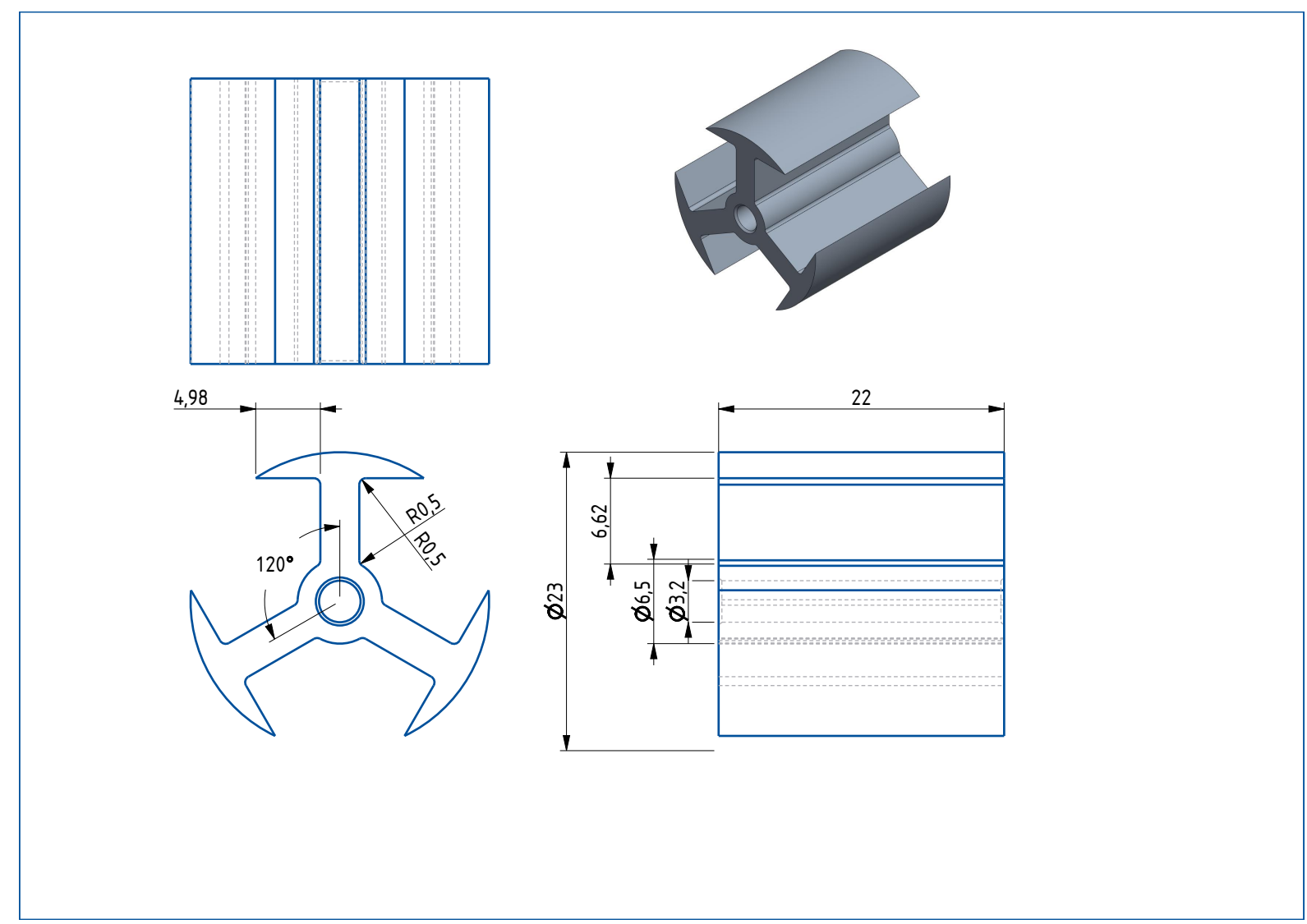

Motor Rotor 3D CAD Drawing 

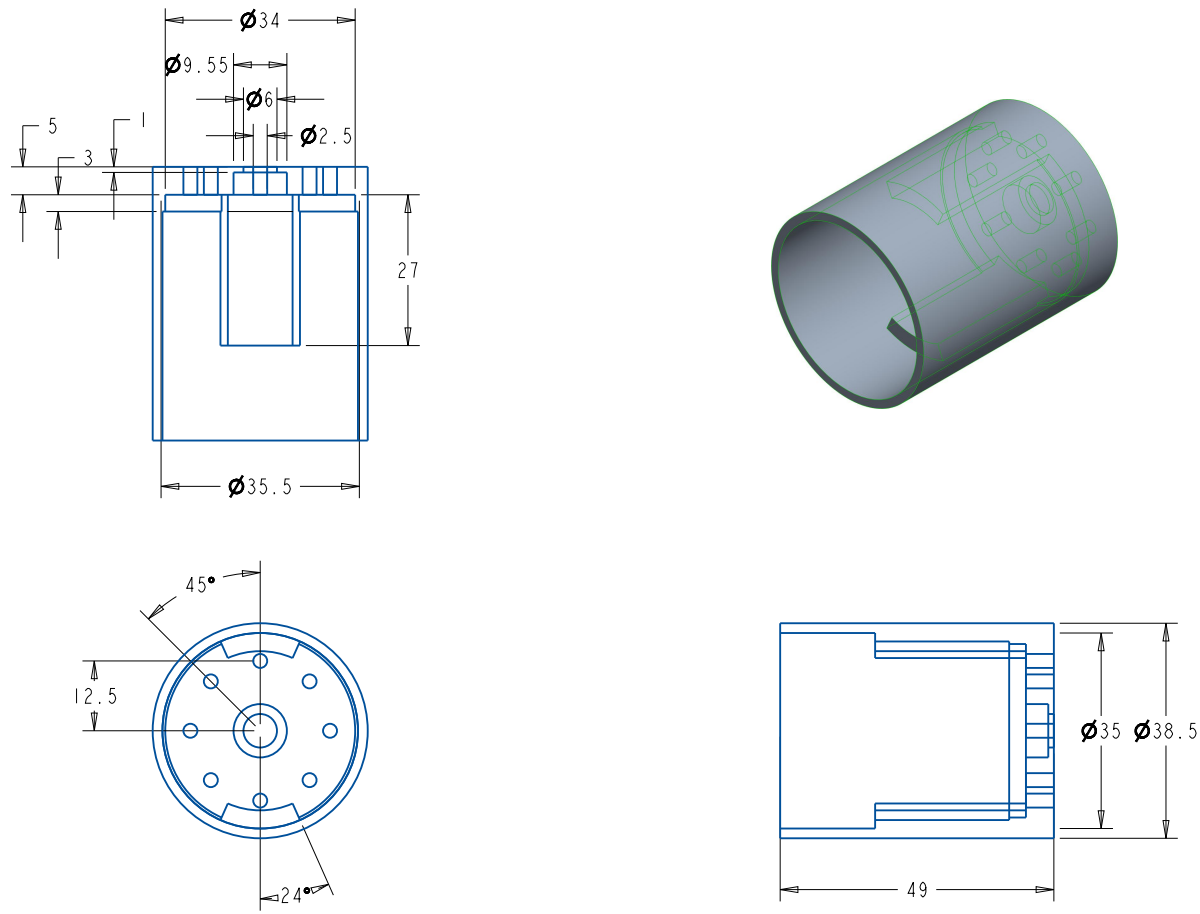

Motor Stator 3D CAD Drawing 

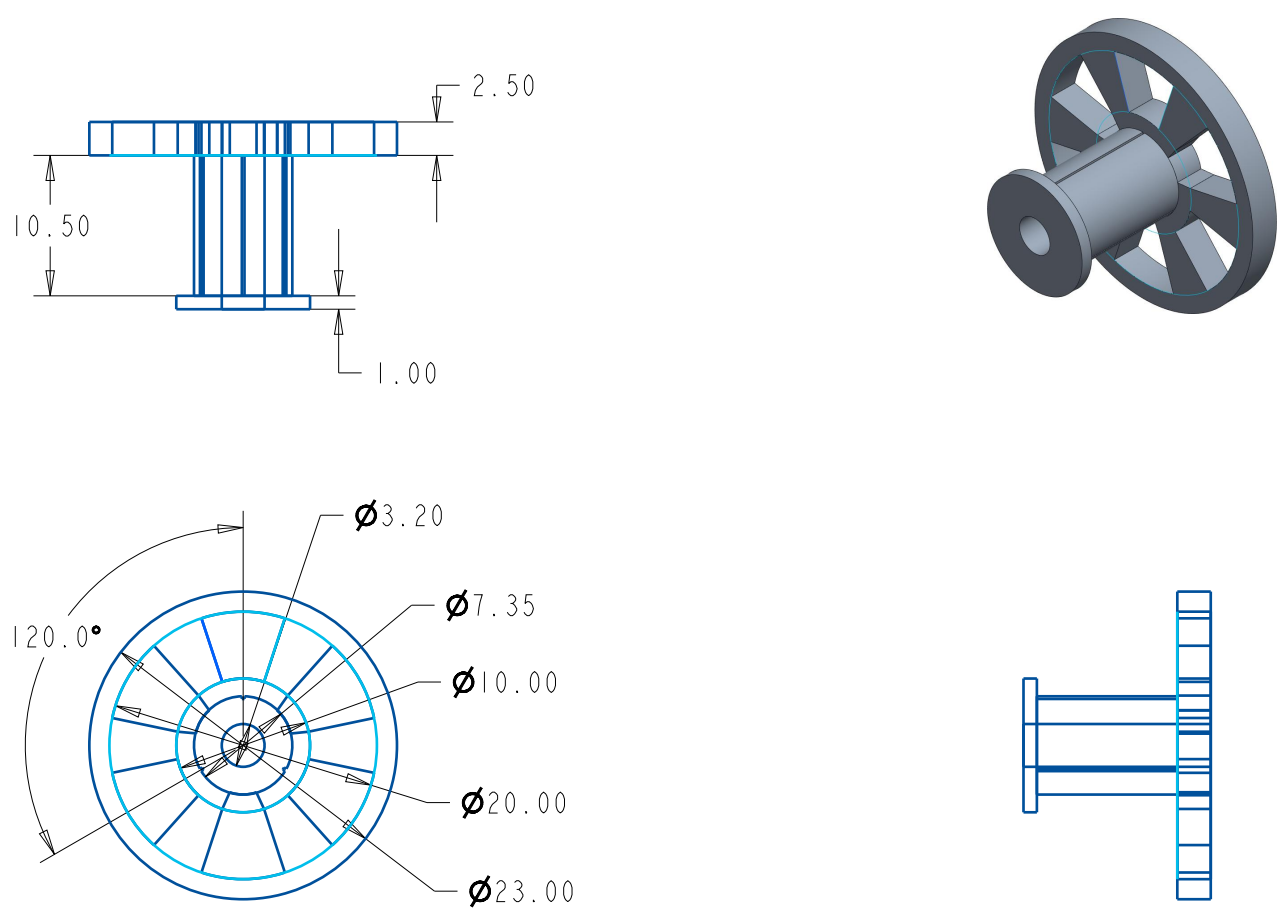

Motor Commutator 3D CAD Drawing 


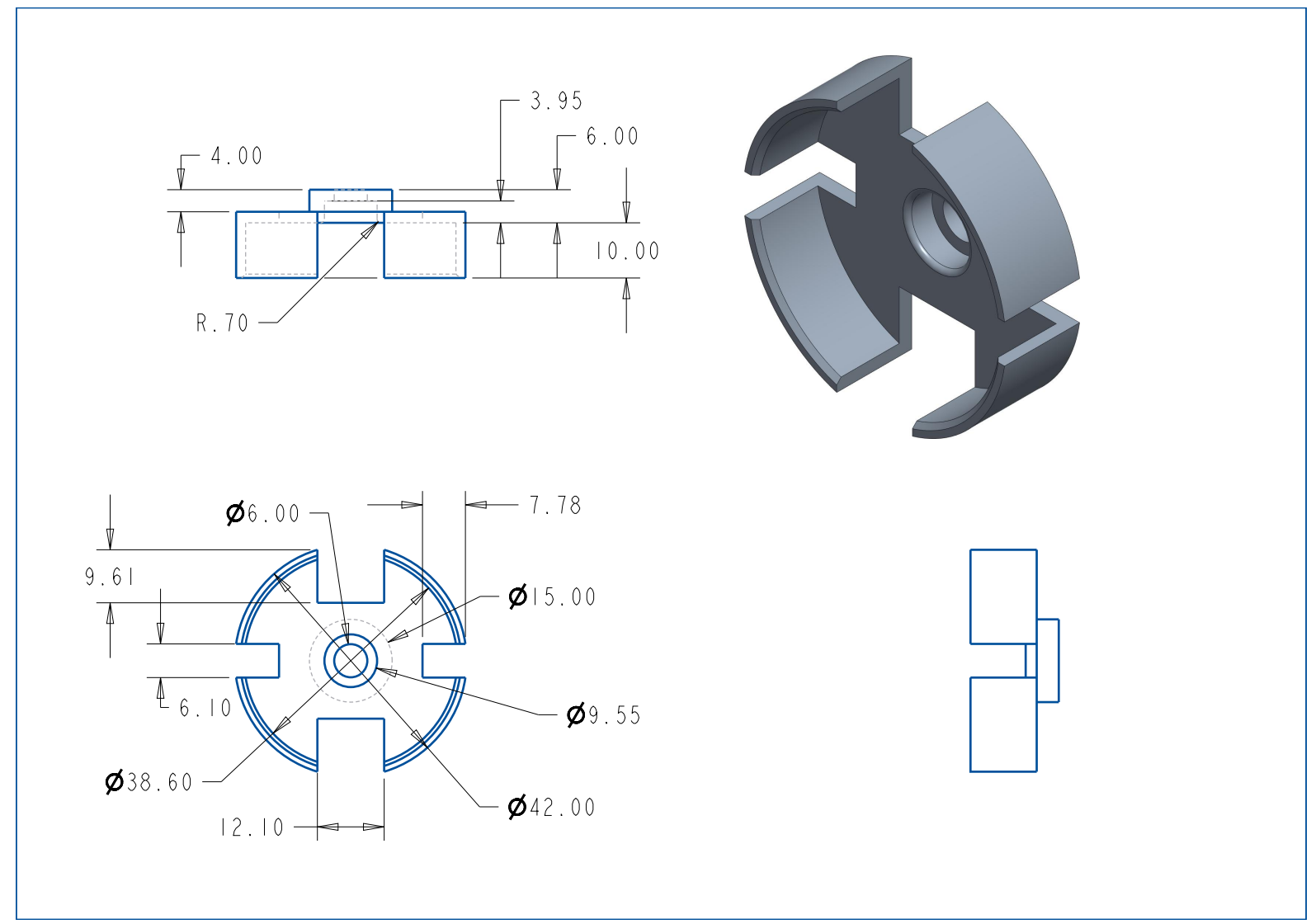

Motor End-Cab 


\section{Bibliography}

[1] Ian Gibson, David W Rosen, and Brent Stucker. Design for additive manufacturing. In Additive Manufacturing Technologies, pages 299-332. Springer, 2010.

[2] Amit Joe Lopes, Eric MacDonald, and Ryan B Wicker. Integrating stereolithography and direct print technologies for 3d structural electronics fabrication. Rapid Prototyping Journal, 2012.

[3] Michele Garibaldi, Ian Ashcroft, Marco Simonelli, and Richard Hague. Metallurgy of high-silicon steel parts produced using selective laser melting. Acta Materialia, 110:207-216, 2016.

[4] M Garibaldi, I Ashcroft, N Hillier, SAC Harmon, and R Hague. Relationship between laser energy input, microstructures and magnetic properties of selective laser melted fe- $6.9 \%$ wt si soft magnets. Materials Characterization, 143:144-151, 2018.

[5] Andreas Krings, Aldo Boglietti, Andrea Cavagnino, and Steve Sprague. Soft magnetic material status and trends in electric machines. IEEE Transactions on Industrial Electronics, 64(3):2405-2414, 2016.

[6] Nikolaus Urban, Alexander Meyer, Sven Kreitlein, Felix Leicht, and Jörg Franke. Efficient near net-shape production of high energy rare earth magnets by laser beam melting. In Applied Mechanics and Materials, volume 871, pages 137-144. Trans Tech Publ, 2017.

[7] Jacim Jacimovic, Federico Binda, Lorenz G Herrmann, Felix Greuter, Jessica Genta, Micha Calvo, Tomaz Tomse, and Reinhard A Simon. Net shape 3d printed ndfeb permanent magnet. arXiv preprint arXiv:1611.05332, 2016.

[8] Jetpack. 3d printing - 3d technical services. [Online], Sep 2018. [Accessed: July 22, 2019].

[9] Mélanie Despeisse and Tim Minshall. Skills and education for additive manufacturing: A review of emerging issues. In IFIP International Conference on Advances in Production Management Systems, pages 289-297. Springer, 2017. 
[10] Vinod G Gokhare, DN Raut, and DK Shinde. A review paper on 3d-printing aspects and various processes used in the 3d-printing. International Journal of Engineering Research \& Technology, 6(6):953-958, 2017.

[11] Samuel Clark Ligon, Robert Liska, Jurgen Stampfl, Matthias Gurr, and Rolf Mullhaupt. Polymers for 3d printing and customized additive manufacturing. Chemical reviews, 117(15):10212-10290, 2017.

[12] Steven Ashley. Rapid prototyping is coming of age. Mechanical Engineering, 117(7):62, 1995.

[13] Izabela Hager, Anna Golonka, and Roman Putanowicz. 3d printing of buildings and building components as the future of sustainable construction? Procedia Engineering, 151:292-299, 2016.

[14] Roberto Soler, Claudius Füllhase, Cesar Santos, and Karl-Erik Andersson. Development of bladder dysfunction in a rat model of dopaminergic brain lesion. Neurourology and urodynamics, 30(1):188-193, 2011.

[15] Anthony Atala, F Kurtis Kasper, and Antonios G Mikos. Engineering complex tissues. Science translational medicine, 4(160):160rv12-160rv12, 2012.

[16] Helena Dodziuk. Applications of 3d printing in healthcare. Kardiochirurgia i torakochirurgia polska= Polish journal of cardio-thoracic surgery, 13(3):283, 2016.

[17] Jeremy Hsu. 3d printing aims to deliver organs on demand. LiveScience, September, 2013.

[18] Brian Betts. Bringing the factory home [personal fabrication technology]. Engineering \& technology, 5(8):56-58, 2010.

[19] Dana Goldberg. History of 3d printing. [Online], 2018. [Accessed: July 18, 2019].

[20] Jean-Pierre Kruth, X Wang, Tahar Laoui, and Ludo Froyen. Lasers and materials in selective laser sintering. Assembly Automation, 23(4):357-371, 2003.

[21] Luke Dormehl. The brief but building history of 3d printing. [Online], 2019. [Accessed: July 10, 2019].

[22] Shapeways. 3d printing. [Online], 2019. [Accessed: November 13, 2019].

[23] Robin PG Tech, Jan-Peter Ferdinand, and Martina Dopfer. Open source hardware startups and their communities. In The decentralized and networked future of value creation, pages 129-145. Springer, 2016.

[24] Paul Marks. 3d printing takes off with the world's first printed plane, 2011.

[25] Chitra Sethi. Coast to coast in a printed hybrid. Mechanical Engineering-CIME, 136(5):12-14, 2014. 
[26] Mark Richardson, Frank Will, and Robbie Napper. Car design for distributed microfactory production. In 37th Australian Transport Research Forum (ATRF), Sidney, Yeni Güney Galler, Avustralya, 2015.

[27] Neil Leach. 3d printing in space. Architectural Design, 84(6):108-113, 2014.

[28] Mary J Werkheiser, Jason Dunn, Michael P Snyder, Jennifer Edmunson, Kennethe Cooper, and Mallory M Johnston. 3d printing in zero-g iss technology demonstration. In AIAA SPACE 2014 Conference and Exposition, page 4470, 2014.

[29] Carl Schubert, Mark C Van Langeveld, and Larry A Donoso. Innovations in 3d printing: a 3d overview from optics to organs. British Journal of Ophthalmology, 98(2):159-161, 2014.

[30] Silvia Benvenuti, Fabio Ceccanti, and Xavier De Kestelier. Living on the moon: topological optimization of a 3d-printed lunar shelter. Nexus Network Journal, 15(2):285-302, 2013.

[31] Matthew B Hoy. 3d printing: making things at the library. Medical reference services quarterly, 32(1):9399, 2013.

[32] 3dprinting. What is 3d printing? [Online], 2019. [Accessed: November 22, 2019].

[33] Martin Baumers, Chris Tuck, Ricky Wildman, Ian Ashcroft, and Richard Hague. Shape complexity and process energy consumption in electron beam melting: a case of something for nothing in additive manufacturing? Journal of Industrial Ecology, 21(S1):S157-S167, 2017.

[34] Douglas S Thomas and Stanley W Gilbert. Costs and cost effectiveness of additive manufacturing. NIST special publication, 1176:12, 2014.

[35] Adriano Ambrosi and Martin Pumera. 3d-printing technologies for electrochemical applications. Chemical Society Reviews, 45(10):2740-2755, 2016.

[36] Hoejin Kim, Yirong Lin, and Tzu-Liang Bill Tseng. A review on quality control in additive manufacturing. Rapid Prototyping Journal, 2018.

[37] Vipul Gupta, Pavel Nesterenko, and Brett Paull. An introduction to 3d printing. 2019.

[38] Miss Prachi More. Making the digital real.

[39] Anna Kaziunas France. Make: 3D printing: the essential guide to 3D printers. Maker Media, Inc., 2013.

[40] Leonidas Gargalis, Vincenzo Madonna, Paolo Giangrande, Mark Hardy, Ian Ashcroft, Michael Galea, and Richard Hague. 3d printing as a technology enabler for electrical machines: Manufacturing and testing of 
a salient pole rotor for srm. In 2020 International Conference on Electrical Machines (ICEM), volume 1, pages 12-18. IEEE, 2020.

[41] Max Murphy. 3d printing tradeoffs and optimization. [Online], August 2015. [Accessed: July 19, 2020].

[42] All3DP. Types of 3d printing technology. [Online], February 2020. [Accessed: July 22, 2019].

[43] j Karthikeyan. The advantages and disadvantages of the cold spray coating process. In The cold spray materials deposition process, pages 62-71. Elsevier, 2007.

[44] Ian Gibson, David Rosen, Brent Stucker, and Mahyar Khorasani. Directed energy deposition. In Additive Manufacturing Technologies, pages 285-318. Springer, 2021.

[45] Tim A Osswald, John Puentes, and Julian Kattinger. Fused filament fabrication melting model. Additive Manufacturing, 22:51-59, 2018.

[46] Edward A Sells. Towards a self-manufacturing rapid prototyping machine. PhD thesis, University of Bath Bath, 2009.

[47] BM Tymrak, Megan Kreiger, and Joshua M Pearce. Mechanical properties of components fabricated with open-source 3-d printers under realistic environmental conditions. Materials \& Design, 58:242-246, 2014.

[48] Joe Hiemenz. 3d printing with fdm: How it works. Stratasys Inc, 1:1-5, 2011.

[49] Michael Chapiro. Current achievements and future outlook for composites in 3d printing. Reinforced Plastics, 60(6):372-375, 2016.

[50] i.materialise. 3d printing technologies. [Online], n.d. [Accessed: July 31, 2020].

[51] M Kunieda. Manufacturing of laminated deep drawing dies by laser beam cutting. Proc. of ICTP, 1984, $1: 520,1984$.

[52] André Dolenc et al. An overview of rapid prototyping technologies in manufacturing. Citeseer, 1994.

[53] Elizabeth Palermo. What is laminated object manufacturing? [Online], 2013. [Accessed: November 10, 2019].

[54] Osama Abdulhameed, Abdulrahman Al-Ahmari, Wadea Ameen, and Syed Hammad Mian. Additive manufacturing: Challenges, trends, and applications. Advances in Mechanical Engineering, 11(2):1687814018822880, 2019. 
[55] Alkaios Bournias Varotsis. Introduction to fdm 3d printing. URL: https://www. 3dhubs. com/knowledgebase/introduction-fdm-3d-printing.

[56] T. S. Srivatsan and T. S. Sudarshan. Additive manufacturing: innovations, advances, and applications. CRC Press/Taylor Francis Group, 2016.

[57] Prashanth Konda Gokuldoss, Sri Kolla, and Jürgen Eckert. Additive manufacturing processes: Selective laser melting, electron beam melting and binder jettingâselection guidelines. Materials, 10(6):672, 2017.

[58] Engineering product design. Direct metal laser sintering. [Online], 2019. [Accessed: April 10, 2020].

[59] Amit Bandyopadhyay and Susmita Bose. Additive manufacturing. CRC press, 2019.

[60] Kıvılcım Ersoy and Berk Barış Çelik. Utilization of additive manufacturing to produce tools. In Design Engineering and Manufacturing. IntechOpen, 2019.

[61] Anatolii Papyrin, Vladimir Kosarev, Sergey Klinkov, Anatolii Alkhimov, and Vasily M Fomin. Cold spray technology. Elsevier, 2006.

[62] TS Srivatsan and TS Sudarshan. Additive manufacturing: innovations, advances, and applications. CRC Press, 2015.

[63] Manu Srivastava, Sandeep Rathee, Sachin Maheshwari, and TK Kundra. Additive Manufacturing: Fundamentals and Advancements. CRC Press, 2019.

[64] Phuong Vo, Stephen Yue, and Wilson Wong. Is cold spray additive manufacturing?, 2020.

[65] Hongyi Yang, Jingying Charlotte Lim, Yuchan Liu, Xiaoying Qi, Yee Ling Yap, Vishwesh Dikshit, Wai Yee Yeong, and Jun Wei. Performance evaluation of projet multi-material jetting 3d printer. Virtual and physical prototyping, 12(1):95-103, 2017.

[66] Shuo Yin, Pasquale Cavaliere, Barry Aldwell, Richard Jenkins, Hanlin Liao, Wenya Li, and Rocco Lupoi. Cold spray additive manufacturing and repair: Fundamentals and applications. Additive Manufacturing, 21:628-650, 2018.

[67] Wenya Li, Kang Yang, Shuo Yin, Xiawei Yang, Yaxin Xu, and Rocco Lupoi. Solid-state additive manufacturing and repairing by cold spraying: A review. Journal of Materials Science \& Technology, 34(3):440457, 2018.

[68] Wei Tong. Mechanical design of electric motors. CRC press, 2014. 
[69] Sang-Hoon Kim. Electric motor control: DC, AC, and BLDC motors. Elsevier, 2017.

[70] Allan R Hambley, Narendra Kumar, and Ashish R Kulkarni. Electrical engineering: principles and applications. Pearson Prentice Hall Upper Saddle River, NJ, 2008.

[71] Giorgio Rizzoni and James Kearns. Principles and applications of electrical engineering. McGraw-Hill (London), 2003.

[72] Continental Fan. Exploded-view-of-dc-brushed-motor. [Online], September 2019. [Accessed: July 10, 2020].

[73] Stephen Chapman. Electric machinery fundamentals. Tata McGraw-Hill Education, 2005.

[74] Electronics Tutorials. Electrical dc motors. [Online], n.d. [Accessed: July 10, 2020].

[75] Kevin Lynch, Nicholas Marchuk, and Matthew Elwin. Embedded computing and mechatronics with the PIC32 microcontroller. Newnes, 2015.

[76] Austin Hughes and Bill Drury. Electric motors and drives: fundamentals, types and applications. Newnes, 2019.

[77] Matt. Designing with d.c. motors. [Online], 1999. Accessed: [November 25, 2019].

[78] Fritz Faulhaber. Motor calculations for coreless brush dc motors. [Online], 2009. [Accessed: August 01, 2020].

[79] Francesco Cupertino, Riccardo Leuzzi, Vito Giuseppe Monopoli, and Giuseppe Leonardo Cascella. Design procedure for high-speed pm motors aided by optimization algorithms. Machines, 6(1):5, 2018.

[80] ML Wang and G Wang. Electromagnetic sensors for assessing and monitoring civil infrastructures. In Sensor Technologies for Civil Infrastructures, pages 238-264. Elsevier, 2014.

[81] Rainer Hilzinger and Werner Rodewald. Magnetic materials: fundamentals, products, properties, applications. Vacuumschmelze, 2013.

[82] S Palagummi and F-G Yuan. Magnetic levitation and its application for low frequency vibration energy harvesting. In Structural Health Monitoring (SHM) in Aerospace Structures, pages 213-251. Elsevier, 2016.

[83] Bunting. Design considerations. [Online], n.d. [Accessed: August 06, 2020]. 
[84] Integrated Magnetics. Magnet design, engineering manufacturing considerations. [Online], n.d. [Accessed: August 2, 2020].

[85] David Lovejoy. Methods of magnetizing components and materials for magnetic particle inspection. In Magnetic Particle Inspection, pages 57-85. Springer, 1993.

[86] First4magnets. How are samarium cobalt magnets made? [Online], 2020. [Accessed: February10, 2020].

[87] Emagnets Uk. How neodymium magnets are made. [Online], n.d. [Accessed: January 10, 2019].

[88] Inc. Dura Magnetics. Magnetic saturation. [Online], n.d. [Accessed: July 23, 2020].

[89] Nondestructive Testing. Magnetization of ferromagnetic materials. [Online], n.d. [Accessed: July 31, 2020].

[90] C Huber, C Abert, F Bruckner, M Groenefeld, O Muthsam, S Schuschnigg, K Sirak, R Thanhoffer, I Teliban, C Vogler, et al. 3d print of polymer bonded rare-earth magnets, and $3 \mathrm{~d}$ magnetic field scanning with an end-user 3d printer. Applied Physics Letters, 109(16):162401, 2016.

[91] Jennifer Chu. Magnetic 3d printed structures. [Online], June 2018. [Accessed: July 12, 2019].

[92] M Parans Paranthaman, Christopher S Shafer, Amy M Elliott, Derek H Siddel, Michael A McGuire, Robert M Springfield, Josh Martin, Robert Fredette, and John Ormerod. Binder jetting: A novel ndfeb bonded magnet fabrication process. Jom, 68(7):1978-1982, 2016.

[93] Kinjal Gandha, Ling Li, IC Nlebedim, Brian K Post, Vlastimil Kunc, Brian C Sales, James Bell, and M Parans Paranthaman. Additive manufacturing of anisotropic hybrid ndfeb-smfen nylon composite bonded magnets. Journal of Magnetism and Magnetic Materials, 467:8-13, 2018.

[94] Iulian Antoniac, Diana Popescu, Aurelian Zapciu, Aurora Antoniac, Florin Miculescu, and Horatiu Moldovan. Magnesium filled polylactic acid (pla) material for filament based 3d printing. Materials, 12(5):719, 2019.

[95] Vijay Raskar. Magnetic material. [Online], September 2012. [Accessed: July 13, 2020].

[96] Vijay Raskar. Magnetic material. [Online], September 2012. (Date last accessed 07-March-2020).

[97] H Shokrollahi and K Janghorban. Soft magnetic composite materials (smcs). Journal of Materials Processing Technology, 189(1-3):1-12, 2007.

[98] Philip Beckley. Electrical steels for rotating machines. Number 37. IET, 2002. 
[99] Richard M Bozorth. Ferromagnetism. Ferromagnetism, by Richard M. Bozorth, pp. 992. ISBN 0-78031032-2. Wiley-VCH, August 1993., page 992, 1993.

[100] Gaoyuan Ouyang, Xi Chen, Yongfeng Liang, Chad Macziewski, and Jun Cui. Review of fe-6.5 wt\% si high silicon steelâa promising soft magnetic material for sub-khz application. Journal of Magnetism and Magnetic Materials, 2019.

[101] Sumitomo Electric. Powder metal products division. [Online], n.d. [Accessed: July 10, 2020].

[102] Santosh Kulkarni, Dai Li, Ningning Wang, Saibal Roy, Cian O Mathuna, George Young, and Paul McCloskey. Low loss magnetic thin films for off-line power conversion. IEEE transactions on Magnetics, 50(4):1-4, 2014.

[103] Ansar Masood, V. StrÃđIm, L. Belova, K. V. Rao, and J. Ãgren. Effect of ni-substitution on glass forming ability, mechanical, and magnetic properties of febnby bulk metallic glasses. Journal of Applied Physics, 113(1):013505, 2013.

[104] Karl Ackland, Ansar Masood, Santosh Kulkarni, and Plamen Stamenov. Ultra-soft magnetic co-fe-b-si-nb amorphous alloys for high frequency power applications. AIP Advances, 8(5):056129, 2018.

[105] Bethel Afework. Electromagnetic induction. [Online], April 2020. [Accessed: August 04, 2020].

[106] E4U. Electromagnetic theory. [Online], July 2018. [Accessed: August 04, 2020].

[107] Ajay Di sharma. Electromagnetism. [Online], July 2020. [Accessed: August 04, 2020].

[108] R. Nave. Faraday's law. [Online], n.d. [Accessed: August 04, 2020].

[109] Michele Garibaldi, Chris Gerada, and Richard Hague. Laser additive manufacturing of soft magnetic cores for rotating electrical machinery: materials development and part design. $\mathrm{PhD}$ thesis, University of Nottingham, 2018.

[110] Nicole C Benack, Tony Wang, Kyle Matthews, and Mitra L Taheri. Additive manufacturing methods for soft magnetic composites (smcs). Microscopy and Microanalysis, 24(S1):1066-1067, 2018.

[111] Kamran Aamir Mumtaz and Neil Hopkinson. Laser melting functionally graded composition of waspaloy® and zirconia powders. Journal of materials science, 42(18):7647-7656, 2007.

[112] Xiaoshan Yang, Xiufang Cui, Guo Jin, Jinna Liu, Yanbo Chen, and Zhe Liu. Soft magnetic property of (fe60co35ni5) 78 si6b12cu1mo3 alloys by laser additive manufacturing. Journal of Magnetism and Magnetic Materials, 466:75-80, 2018. 
[113] T Borkar, R Conteri, X Chen, RV Ramanujan, and R Banerjee. Laser additive processing of functionallygraded fe-si-b-cu-nb soft magnetic materials. Materials and Manufacturing Processes, 32(14):15811587, 2017.

[114] Lei Yan, Xueyang Chen, Yunlu Zhang, Joseph W Newkirk, and Frank Liou. Fabrication of functionally graded ti and $\gamma$-tial by laser metal deposition. JOM, 69(12):2756-2761, 2017.

[115] Varun Chaudhary, Nartu Mohan Sai Kiran Kumar Yadav, Srinivas Aditya Mantri, Sriswaroop Dasari, Abhinav Jagetia, RV Ramanujan, and R Banerjee. Additive manufacturing of functionally graded co-fe and ni-fe magnetic materials. Journal of Alloys and Compounds, page 153817, 2020.

[116] Baicheng Zhang, Nour-Eddine Fenineche, Hanlin Liao, and Christian Coddet. Magnetic properties of insitu synthesized feni3 by selective laser melting fe-80\% ni powders. Journal of Magnetism and Magnetic Materials, 336:49-54, 2013.

[117] CV Mikler, V Chaudhary, V Soni, B Gwalani, RV Ramanujan, and R Banerjee. Tuning the phase stability and magnetic properties of laser additively processed fe-30at\% ni soft magnetic alloys. Materials Letters, 199:88-92, 2017.

[118] H Schönrath, M Spasova, SO Kilian, R Meckenstock, G Witt, JT Sehrt, and M Farle. Additive manufacturing of soft magnetic permalloy from fe and ni powders: Control of magnetic anisotropy. Journal of Magnetism and Magnetic Materials, 478:274-278, 2019.

[119] CV Mikler, V Chaudhary, T Borkar, V Soni, D Jaeger, X Chen, R Contieri, RV Ramanujan, and R Banerjee. Laser additive manufacturing of magnetic materials. Jom, 69(3):532-543, 2017.

[120] CV Mikler, V Chaudhary, T Borkar, V Soni, D Choudhuri, RV Ramanujan, and R Banerjee. Laser additive processing of ni-fe-v and ni-fe-mo permalloys: microstructure and magnetic properties. Materials Letters, 192:9-11, 2017.

[121] ProtoPlant. Magnetic iron pla. [Online], n.d. [Accessed: June 13, 2021].

[122] Huaishu Peng, François Guimbretière, James McCann, and Scott Hudson. A 3d printer for interactive electromagnetic devices. In Proceedings of the 29th Annual Symposium on User Interface Software and Technology, pages 553-562. ACM, 2016.

[123] Valkyrie Savage, Colin Chang, and Björn Hartmann. Sauron: embedded single-camera sensing of printed physical user interfaces. In Proceedings of the 26th annual ACM symposium on User interface software and technology, pages 447-456. ACM, 2013. 
[124] Jonathan Hook, Thomas Nappey, Steve Hodges, Peter Wright, and Patrick Olivier. Making 3d printed objects interactive using wireless accelerometers. In Proceedings of the extended abstracts of the 32nd annual ACM conference on Human factors in computing systems, pages 1435-1440. ACM, 2014.

[125] Wei Gao, Yunbo Zhang, Diogo C Nazzetta, Karthik Ramani, and Raymond J Cipra. Revomaker: Enabling multi-directional and functionally-embedded 3d printing using a rotational cuboidal platform. In Proceedings of the 28th Annual ACM Symposium on User Interface Software \& Technology, pages 437-446. ACM, 2015.

[126] Valkyrie Savage, Sean Follmer, Jingyi Li, and Björn Hartmann. Makers' marks: Physical markup for designing and fabricating functional objects. In Proceedings of the 28th Annual ACM Symposium on User Interface Software \& Technology, pages 103-108. ACM, 2015.

[127] Karl Willis, Eric Brockmeyer, Scott Hudson, and Ivan Poupyrev. Printed optics: 3d printing of embedded optical elements for interactive devices. In Proceedings of the 25th annual ACM symposium on User interface software and technology, pages 589-598. ACM, 2012.

[128] Yoshio Ishiguro and Ivan Poupyrev. 3d printed interactive speakers. In Proceedings of the SIGCHI Conference on Human Factors in Computing Systems, pages 1733-1742. ACM, 2014.

[129] Marynel Vázquez, Eric Brockmeyer, Ruta Desai, Chris Harrison, and Scott E Hudson. 3d printing pneumatic device controls with variable activation force capabilities. In Proceedings of the 33rd Annual ACM Conference on Human Factors in Computing Systems, pages 1295-1304. ACM, 2015.

[130] Robert MacCurdy, Robert Katzschmann, Youbin Kim, and Daniela Rus. Printable hydraulics: A method for fabricating robots by $3 \mathrm{~d}$ co-printing solids and liquids. In 2016 IEEE International Conference on Robotics and Automation (ICRA), pages 3878-3885. IEEE, 2016.

[131] Martin Schmitz, Mohammadreza Khalilbeigi, Matthias Balwierz, Roman Lissermann, Max Mühlhäuser, and Jürgen Steimle. Capricate: A fabrication pipeline to design and 3d print capacitive touch sensors for interactive objects. In Proceedings of the 28th Annual ACM Symposium on User Interface Software \& Technology, pages 253-258. ACM, 2015.

[132] Matthew Humphries. Voxel8 3d printer can print a complete quadcopter, including the circuits. [Online], 2015. [Accessed: November 10, 2019].

[133] CY Kim, A Cuaron, MA Perez, D Espalin, E MacDonald, and RB Wicker. Cooperative fabrication methodology for embedding wireon curved surfaces. 2015. 
[134] Jacob Bayless, Mo Chen, and Bing Dai. Wire embedding 3d printer. Engineering Physics Department, University of British Columbia, 2010.

[135] bas de bruijn. Additive wire-laying. [Online], 2014. [Accessed: July 10, 2019].

[136] Mark A Skylar-Scott, Suman Gunasekaran, and Jennifer A Lewis. Laser-assisted direct ink writing of planar and 3d metal architectures. Proceedings of the National Academy of Sciences, 113(22):6137-6142, 2016.

[137] Christoph Laimer. 600 watt, halbach brushless motor. [Online], 2017. Accessed: [November 28, 2019].

[138] Edward A Sells. Towards a self-manufacturing rapid prototyping machine. PhD thesis, University of Bath Bath, 2009.

[139] Gaoyuan Ouyang, Brandt Jensen, Wei Tang, Kevin Dennis, Chad Macziewski, Srinivasa Thimmaiah, Yongfeng Liang, and Jun Cui. Effect of wheel speed on magnetic and mechanical properties of melt spun fe-6.5 wt.\% si high silicon steel. Aip Advances, 8(5):056111, 2018.

[140] A Sova, S Grigoriev, A Okunkova, and I Smurov. Potential of cold gas dynamic spray as additive manufacturing technology. The International Journal of Advanced Manufacturing Technology, 69(9-12):22692278, 2013.

[141] Mininpro. Hobby motor inertia dyno v1. [Online], 2019. [Accessed: November 18, 2019].

[142] Electrical4u. Types of dc motors and their applications. [Online], 2019. [Accessed: July 25, 2019].

[143] Edson Costa Santos, Masanari Shiomi, Kozo Osakada, and Tahar Laoui. Rapid manufacturing of metal components by laser forming. International Journal of Machine Tools and Manufacture, 46(12-13):14591468, 2006.

[144] ICAMP. Innovation center for advanced manufacturing and production, 2019. [Accessed: July 18, 2019].

[145] Girdhar Gyani and Om Parkash. Design and technological aspects of printed motor armature. IEE-IERE Proceedings-India, 15(2):64-68, 1977.

[146] M-C Tsai and L-Y Hsu. Design of a miniature axial-flux spindle motor with rhomboidal pcb winding. IEEE transactions on magnetics, 42(10):3488-3490, 2006.

[147] Siamak Atar. Direct drive stacked motor acuator, March 26 2015. US Patent App. 14/489,985. 
[148] MGchemicals. Instructions for the basic positive prototyping process. [Online], 2019. [Accessed: November 10, 2019].

[149] Alex Ellery and Abdurrazag Elaskri. Steps toward self-assembly of lunar structures from modules of 3dprinted in-situ resources. 70th International Astronautical Congress (IAC), Washington D.C., United States, 2019.

[150] RC planet. Dynamite brushed motor 20-turn dyn1171. [online] Available at: https://www.rcplanet.com/motors/car-truck-motors/dynamite-brushed-motor-20-turn-dyn1171/, 2021. [Accessed 31 March 2021].

[151] Precisionmicrodrives.com. A short illustrated primer on brushed de motors - precision microdrives. [Online] Available at: https://www.precisionmicrodrives.com/content/a-short-illustrated-primer-on-brusheddc-motors/, 2021. [Accessed 31 March 2021].

[152] BR Gupta. Generation of electrical energy. S. Chand Publishing, 2017.

[153] Circuit Globe. What is lap and wave winding? definition types - circuit globe. [Online] Available at: https://circuitglobe.com/lap-and-wave-winding.html, 2018. [Accessed 31 March 2021]. 